\title{
Designing Faster CMOS Sub-threshold Circuits Utilizing Channel Length Manipulation
}

by

Farhad Ramezankhani, B. Sc., M. A. Sc.

A thesis submitted to the Faculty of Graduate and Postdoctoral Affairs in partial fulfillment of the requirements for the degree of

\section{Master of Applied Science}

\author{
in \\ Electrical and Computer Engineering
}

Ottawa-Carleton Institute for Electrical and Computer Engineering

Carleton University

Department of Electronics

Ottawa, Ontario, Canada

September 2012

(C) 2012, Farhad Ramezankhani 
Library and Archives

Canada

Published Heritage

Branch

395 Wellington Street

Ottawa ON K1A ON4

Canada
Bibliothèque et

Archives Canada

Direction du

Patrimoine de l'édition

395 , rue Wellington

Ottawa ON K1A ON4

Canada
Your file Votre référence

ISBN: 978-0-494-93510-1

Our file Notre référence

ISBN: 978-0-494-93510-1
NOTICE:

The author has granted a nonexclusive license allowing Library and Archives Canada to reproduce, publish, archive, preserve, conserve, communicate to the public by telecommunication or on the Internet, loan, distrbute and sell theses worldwide, for commercial or noncommercial purposes, in microform, paper, electronic and/or any other formats.

The author retains copyright ownership and moral rights in this thesis. Neither the thesis nor substantial extracts from it may be printed or otherwise reproduced without the author's permission.
AVIS:

L'auteur a accordé une licence non exclusive permettant à la Bibliothèque et Archives Canada de reproduire, publier, archiver, sauvegarder, conserver, transmettre au public par télécommunication ou par l'Internet, prêter, distribuer et vendre des thèses partout dans le monde, à des fins commerciales ou autres, sur support microforme, papier, électronique et/ou autres formats.

L'auteur conserve la propriété du droit d'auteur et des droits moraux qui protege cette thèse. $\mathrm{Ni}$ la thèse ni des extraits substantiels de celle-ci ne doivent être imprimés ou autrement reproduits sans son autorisation.
In compliance with the Canadian Privacy Act some supporting forms may have been removed from this thesis.

While these forms may be included in the document page count, their removal does not represent any loss of content from the thesis.
Conformément à la loi canadienne sur la protection de la vie privée, quelques formulaires secondaires ont été enlevés de cette thèse.

Bien que ces formulaires aient inclus dans la pagination, il n'y aura aucun contenu manquant. 


\section{Abstract}

The Reverse-Short-Channel Effect in MOSFETs decreases the threshold voltage of a transistor at longer channel lengths. Due to the exponential relation between the current and threshold voltage in the sub-threshold region, increasing the channel length may result in a maximum point in the current curve versus the channel length. Increasing the channel length also increases the capacitances involve in the delay. A method based on this behaviour is proposed to find the optimal channel lengths that maximize the Currentover-Capacitance $(\mathrm{CoC})$ of a transistor. The $\mathrm{CoC}$ method is extended to serial and parallel transistor connections. The effectiveness of the $\mathrm{CoC}$ method is verified by incorporating the obtained optimum channel lengths in ring oscillators consisting of Inverter, NAND, NOR, and AOI gates. An improvement of $95 \%$ in the operation frequency is achieved compared to the popular minimum-size sub-threshold circuits. Using the optimum channel lengths in a 32-bit Carry-Look-Ahead adder shows about $50 \%, 20 \%$, and $60 \%$ improvements in the delay, energy, and EDP, respectively compared to the minimum-size version. The method is applied to the TSMC $65 \mathrm{~nm}$, TSMC $90 \mathrm{~nm}$, IBM $130 \mathrm{~nm}$, and TSMC $180 \mathrm{~nm}$ CMOS technologies. 


\section{In the Name of God, \\ the Compassionate, the Merciful}




\section{Acknowledgements}

\section{Whoever doesn't thank others, hasn't indeed thanked God.}

I wish to extend my utmost thanks to my thesis supervisor, Dr. Maitham Shams for his guidance, support and patience. Working with him was a pleasant experience and the time spent with him is an invaluable asset for me.

I would like to thank Professors Calvin Plett, Ralph Mason, Niall Tait, and Mustapha Yagoub for their careful review and contributions to the thesis.

I would like to thank the staff members at the Department of Electronics, especially, Blazenka Power, Anna Lee, Rob Vandusen, Nagui Mikhail, and Scott Bruce.

I would also like to thank my friends Dr. Reza Yousefi, Behzad Yadegari, Morteza Nabavi, Xing Zhou, and Bai Zhanjun for their technical and moral assistance.

I would also like to thank CMC Microsystem and their technology partners for access to the design tools used in the research for this thesis.

On a personal note, I wish to thank my lovely wife and daughter, for their love, patience, and support throughout my studies. 


\section{Dedication}

This dissertation is dedicated to...

my wife, and our lovely daughter

for their unconditional love, patience, and support,

my father's soul,

for believing in me and for his support and encouragement. His absence

is deeply felt. 


\section{Contents}

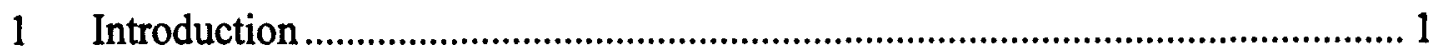

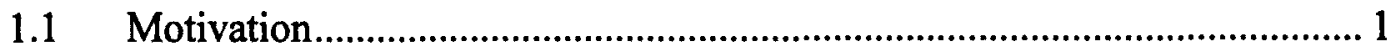

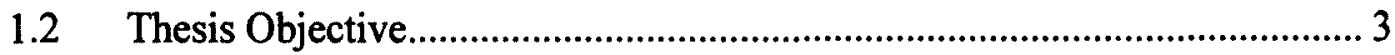

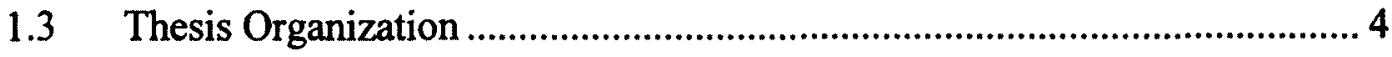

2 Literature Review: ULP and Sub-threshold Circuits ........................................ 5

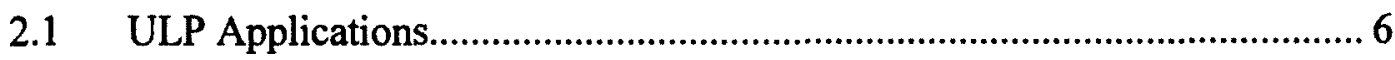

2.2 Why Sub-threshold?............................................................................ 10

2.3 Roadmap of Sub-threshold Circuits Design .............................................. 14

2.4 Chapter Summary ................................................................................ 19

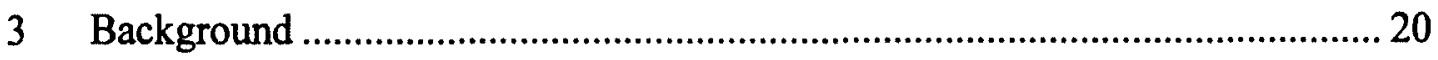

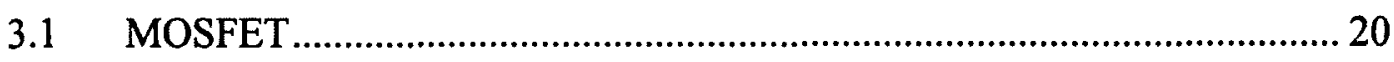

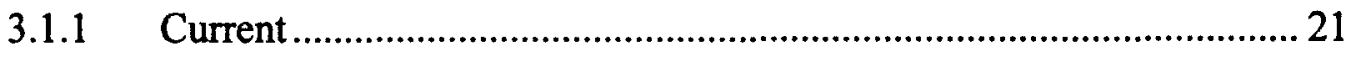

3.1.2 Threshold Voltage .............................................................................. 24

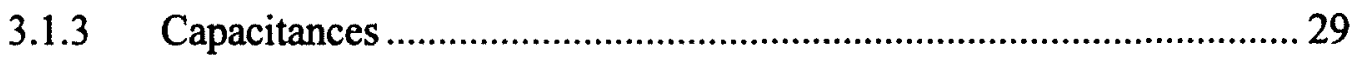

3.1.4 Leakage Currents ............................................................................. 32

3.2 Quality Metrics of a Digital Circuit …………….......................................... 33

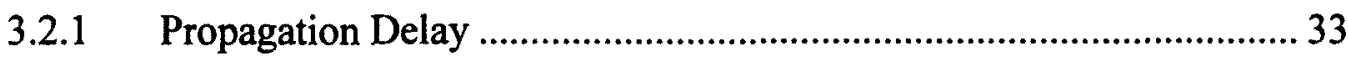

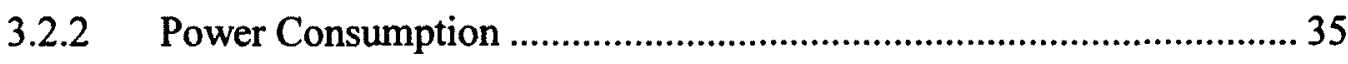

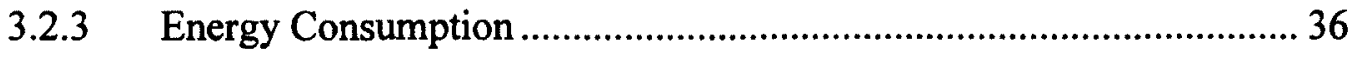

3.2.4 Voltage Transfer Characteristics .......................................................... 38

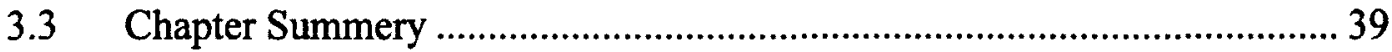

4 MOSFET Behavior in Sub-threshold Region .....................................................4 40

4.1 Threshold Voltage Variation....................................................................... 40

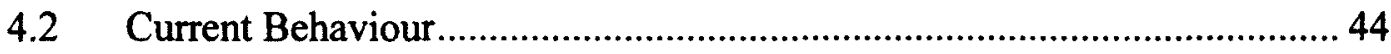




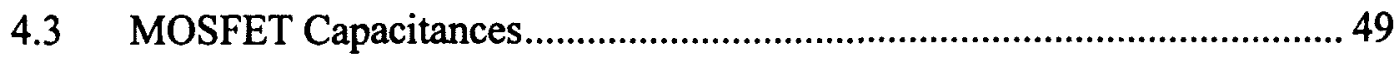

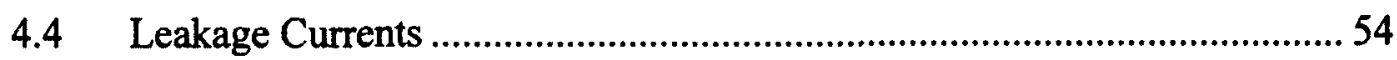

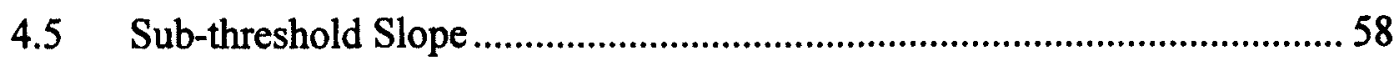

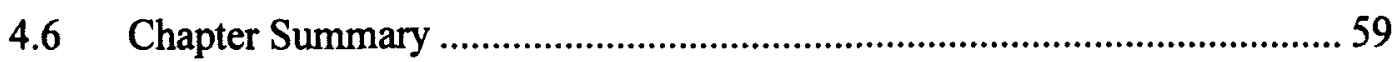

5 Delay Optimization in Sub-threshold Circuits .....................................................6 60

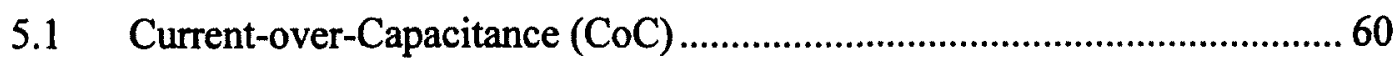

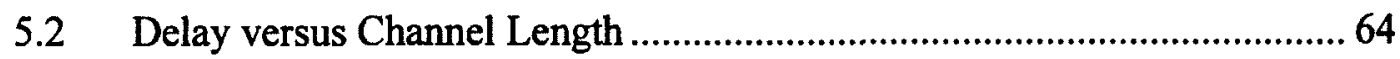

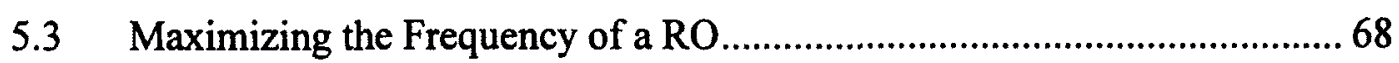

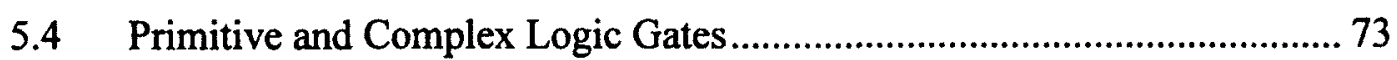

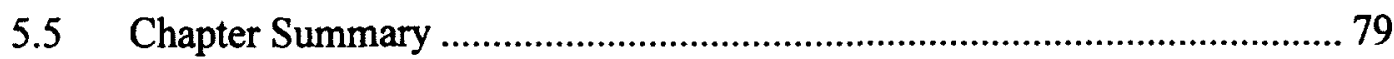

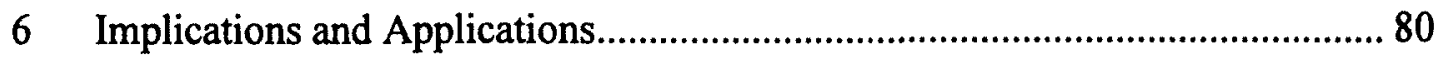

6.1 Increasing $V_{\mathrm{DD}}$ versus Channel-length Manipulation ..................................8 80

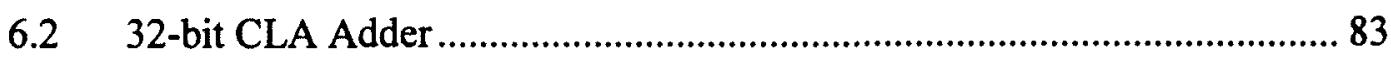

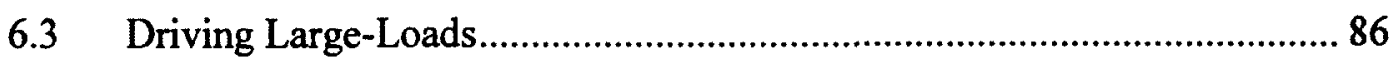

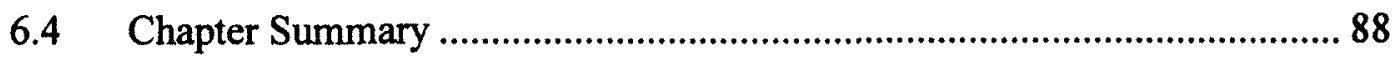

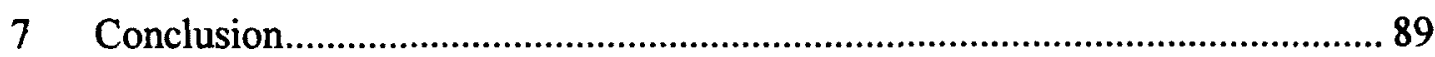

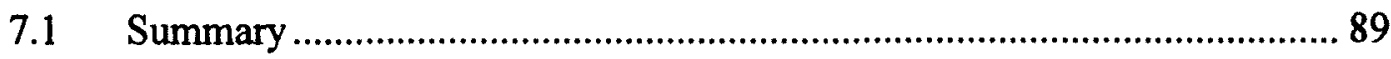

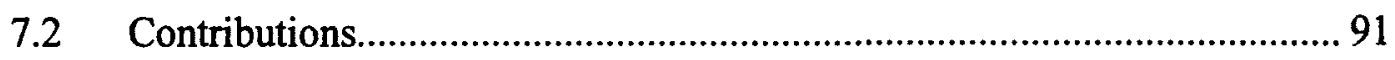

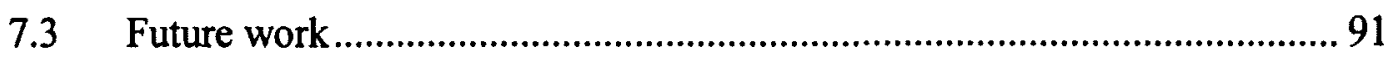

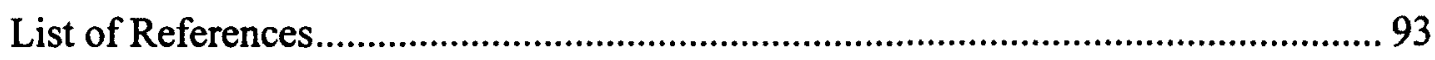




\section{List of Figures}

Figure 2.1 Normalized static current for an inverter versus $V_{\mathrm{DD}}$ in $90 \mathrm{~nm}$ and $130 \mathrm{~nm}$ technologies at minimum sizes.

Figure 2.2 Static and Dynamic energy (a) and total energy (b) versus $V_{D D}$ for 29-inverter $\mathrm{RO}$ in $130 \mathrm{~nm}$ technology at minimum size. 12

Figure 2.3 Energy vs. Frequency (top) and $I_{\text {on }} / I_{\text {off }}$ ratio vs. $V_{\text {DD }}$ (bottom) simulated in $130 \mathrm{~nm}$ technology. 15

Figure 3.1 structure of an n-channel MOSFET (NMOS). 20

Figure 3.2 Current vs. $V_{\mathrm{gs}}$ in logarithmic scale. 23

Figure 3.3 Cross-section of a MOS transistor along the width showing LOCOS (a) and STI (b) isolation and their effect on threshold voltage. 25

Figure 3.4 Charge sharing between source/drain depletion regions and the channel depletion region resulting in threshold roll-off. 26

Figure 3.5 DIBL in a short-channel device. 27

Figure 3.6 HALO doping effects on threshold voltage of short and long-channel transistors. 28

Figure 3.7 MOSFET Capacitances. 29

Figure 3.8 (a) Representation of MOSFET capacitances, (b) decomposition of source/drain junction capacitance to bottom and sidewall components. 29

Figure 3.9 Dependence of gate capacitance of an NMOS transistor to gate voltage........ 31 Figure 3.10 Propagation delay for an inverter driving another inverter with input and output signals approximated as ramps. 34

Figure 3.11 Minimum energy point 37

Figure 3.12 Typical inverter VTC. 38

Figure 4.1 Threshold voltage versus the channel width at $L_{\min }=120 \mathrm{~nm}$ (left), and threshold voltage versus the channel length at $W_{\min }=160 \mathrm{~nm}$ (right) for IBM $130 \mathrm{~nm}$ technology.

Figure 4.2 Threshold voltage versus $W$ at $V_{\mathrm{DD}}=0.2 \mathrm{~V}$ for a PMOS transistor in different technology nodes at $L=L_{\min }$ 
Figure 4.3 Threshold voltage versus $W$ at $V_{\mathrm{DD}}=0.2 \mathrm{~V}$ for an NMOS transistor in different technology nodes at $L=L_{\min }$

Figure 4.4 Threshold voltage versus $L$ at $V_{\mathrm{DD}}=0.2 \mathrm{~V}$ for a PMOS transistor in different technology nodes at $W=W_{\min }$ 43

Figure 4.5 Threshold voltage versus $L$ at $V_{\mathrm{DD}}=0.2 \mathrm{~V}$ for an NMOS transistor in different technology nodes at $W=W_{\min }$

Figure 4.6 Super-threshold current for a PMOS transistor at $V_{\mathrm{DD}}=1 \mathrm{~V}$ versus $L$ and $W$, in IBM 130nm CMOS (NMOS transistor shows the same behavior). 44

Figure 4.7 Top figure shows normalized $1 / L$ and $\exp \left(V_{G S}-V_{t h}\right) / n v_{T}$ ) factors individually plotted versus $L$. The bottom figure shows normalized $1 / L \times \exp \left(V_{G S}-\right.$ $\left.V_{t h}\right) / n v_{T}$ ) and normalized current at $V_{\mathrm{DD}}=0.2 \mathrm{~V}$ for TSMC $65 \mathrm{~nm} \mathrm{LP} \mathrm{CMOS} \mathrm{kit} \mathrm{for} \mathrm{a}$ PMOS-lvt transistor at $W=W_{\min }=120 \mathrm{~nm}$. 45

Figure 4.8 Top figure shows normalized $1 / L$ and $\left.\exp \left(V_{G S}-V_{t h}\right) / n v_{T}\right)$ factors individually plotted versus $L$. The bottom figure shows normalized $1 / L X \exp \left(V_{G S}-\right.$ $\left.V_{t h}\right) / n v_{T}$ ) and normalized current at $V_{\mathrm{DD}}=0.2 \mathrm{~V}$ for TSMC $65 \mathrm{~nm} \mathrm{LP}$ CMOS kit for a PMOS-svt transistor at $W=W_{\min }=120 \mathrm{~nm}$. 46

Figure 4.9 Sub-threshold current versus $W$ in different technology nodes at $V_{\mathrm{DD}}=0.2 \mathrm{~V}$ and $L_{\min }$.

Figure 4.10 Sub-threshold current versus $L$ in different technology nodes at $V_{\mathrm{DD}}=0.2 \mathrm{~V}$ and $W_{\min }$ 48

Figure 4.11 Sub-threshold current versus the channel length for an NMOS transistor in IBM $130 \mathrm{~nm}$ technology at $W_{\min }$. The maximum point becomes smaller as the supply voltage increases.

Figure $4.12 L_{\mathrm{Imax}}$ versus $V_{\mathrm{DD}}$ at $W_{\min }$. 50

Figure 4.13 Gate capacitances versus gate voltage for an NMOS transistor in IBM $130 \mathrm{~nm}$ for two different $V_{\mathrm{DS}}$. $C_{\mathrm{GG}}$ is equal to the sum of the other three capacitances... 51 Figure 4.14 Drain-body junction capacitance versus $V_{\mathrm{DS}}$ for an NMOS in IBM $130 \mathrm{~nm}$ technology at two different $V_{\mathrm{GS}}$. 52 Figure 4.15 Gate and drain capacitances versus the channel width and channel length for an NMOS in IBM $130 \mathrm{~nm}$ at $V_{\mathrm{DS}}=V_{\mathrm{GS}}=0.2 \mathrm{~V}$. 53 
Figure $4.16 C_{\text {total }}$ versus the channel length (left) and versus the channel width (right) for an NMOS in IBM $130 \mathrm{~nm}$ for two sets of voltages that are used in delay modeling and estimation. 53

Figure 4.17 Test benches used for leakage currents measurement. 55

Figure $4.18 I_{\text {off }}$ and $I_{\text {on }}$ versus $L\left(@ W_{\min }\right)$ left figure, and versus $W\left(@ L_{\min }\right)$ right figure for PMOS transistor in IBM $130 \mathrm{~nm}$ technology at $V_{\mathrm{DD}}=0.2 \mathrm{~V}$. 56

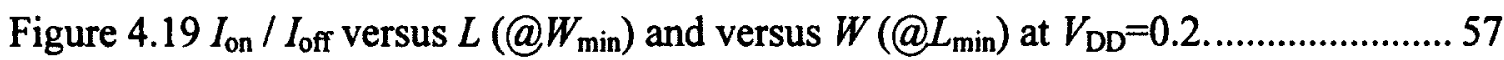
Figure 4.20 Sub-threshold slope versus W (@ $L_{\min }$ ) (left) and versus L (@ $\left.W_{\min }\right)$ (right) for NMOS and PMOS transistors. 58

Figure 5.1 An inverter driving an identical inverter. High-to-low and low-to-high transitions are illustrated.

Figure 5.2 CoC versus $L$ for NMOS and PMOS transistors in IBM $130 \mathrm{~nm}$ technology at $V_{\mathrm{DD}}=0.2 \mathrm{~V}$ 62

Figure 5.3 CoC versus $L$ for PMOS-lvt in TSMC $65 \mathrm{~nm} \mathrm{LP}$ at two different supply voltages. 63

Figure $5.4 L_{\mathrm{COC} \max }$ versus $V_{\mathrm{DD}}$ 64

Figure 5.5 Delay versus $L_{\mathrm{p}}$ and $L_{\mathrm{n}}$ for an inverter driving an identical inverter. 3D plot (top) and contour plot (bottom) simulated in IBM $130 \mathrm{~nm}$ at $V_{\mathrm{DD}}=0.2 \mathrm{~V}$ 65

Figure 5.6 Delay contours versus $L_{\mathrm{p}}$ and $L_{\mathrm{n}}$ in TSMC $65 \mathrm{~nm}$ at $V_{\mathrm{DD}}=0.2 \mathrm{~V}$. 66 Figure 5.7 Delay versus supply voltage measured for three different sets of transistors sizing. 67

Figure 5.8 VTC for an inverter plotted for four sets of channel lengths in $65 \mathrm{~nm}$ at $V_{\mathrm{DD}}=0.2 \mathrm{~V}$. Both NMOS and PMOS transistors are "lvt" types. 71

Figure 5.9 A RO with NAND2 logic gates connected in its worst-case scenario. 73 Figure 5.10 Pull-down (a) and pull-up (b) networks for a NAND2 logic gate connected in the worst case scenario. 74

Figure 5.11 $L_{\mathrm{COCmax}}$ versus $V_{\mathrm{DD}}$ for different connection topologies of transistors used as a building blocks for logic gates 78

Figure 6.1 Energy per operation and frequency vs. $V_{\mathrm{DD}}$ for a $29-\mathrm{INV}$ RO in the $65 \mathrm{~nm}$ technology at $L_{\min }=60 \mathrm{~nm}$. 80 
Figure 6.2 Energy per operation and frequency vs. $V_{\mathrm{DD}}$ for a $29-\mathrm{INV}$ RO in the $65 \mathrm{~nm}$ technology at $W_{\min }=120 \mathrm{~nm}$.

Figure 6.3 Energy per operation and frequency vs. $V_{\mathrm{DD}}$ for a $29-\mathrm{INV}$ RO in the $65 \mathrm{~nm}$ technology at $W_{\min }=120 \mathrm{~nm}$. 83

Figure 6.4 EDP versus $V_{\mathrm{DD}}$ for a 29-INV RO simulated for four different sets of transistor

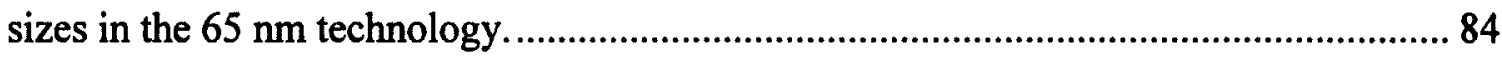

Figure 6.5 Block diagram for a 4-bit CLA adder [15]................................................. 84

Figure 6.6 Driving a large load with a chain of three inverters. ......................................... 87

Figure 6.7 Output signal of a chain driving a large load at $V_{\mathrm{DD}}=0.2 \mathrm{~V}$ and $W_{\min }=120 \mathrm{~nm}$

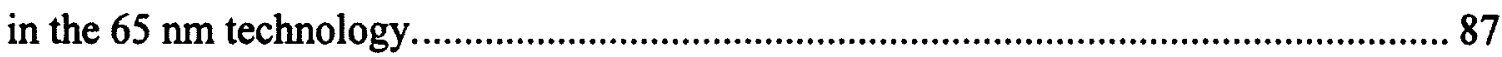




\section{List of Tables}

Table 2.1 Low-power techniques for high-performance digital circuits [19].................... 7

Table 2.2 Micro-sensor networks applications [17] ...................................................... 8

Table 2.3 Example of power harvesting mechanism and their typical power densities..... 9

Table 2.4 Leakage power savings from voltage reduction (constant leakage current)..... 11

Table 2.5 Summary of Sub-threshold papers................................................................... 18

Table 3.1 Approximation for MOSFET capacitances [80]............................................... 31

Table 4.1 The leakage currents for NMOS and PMOS transistors in each technology at their minimum acceptable sizes.................................................................................... 55

Table 5.1 Delay measured for three different sets of transistor sizing at $V_{\mathrm{DD}}=0.2 \mathrm{~V}$....... 67

Table 5.2 Frequency for a RO with 9 and 29 inverters simulated for two different sets of transistor sizing at $V_{\mathrm{DD}}=0.2 \mathrm{~V}$

Table 5.3 Frequency in a 29 inverter RO simulated for three sets of channel lengths at $V_{\mathrm{DD}}=0.2 \mathrm{~V}$. 70

Table 5.4 Frequency for a 29 inverter RO in different supply voltages for three different sets of channel lengths for TSMC $65 \mathrm{~nm}$ LP. 70

Table 5.5 Noise margins for an inverter in three sets of channel length compared to that of the minimum size inverter. Energy per cycle and frequency operation of a 29 inverter RO compared for these four sets of channel lengths. .................................................... 72

Table 5.6 $L_{\mathrm{COCmax}}$ for transistor configuration shown in Figure 5.10 at $V_{\mathrm{DD}}=0.2 \mathrm{~V}$........ 74

Table $5.7 L_{\mathrm{CoCmax}}$ for different combinations of MOSFETs at $V_{\mathrm{DD}}=0.2 \mathrm{~V}$....................... 75

Table 5.8 Simulation results for RO consisting of 29 of each logic gate for four sets of channel lengths at $V_{\mathrm{DD}}=0.2 \mathrm{~V}$.

Table 6.1 Simulation results for a 32-bit CLA adder in the $65 \mathrm{~nm}$ technology at $V_{\mathrm{DD}}=0.2$.

Table 6.2 Simulation results for a three-inverters chain driving a large load at $V_{\mathrm{DD}}=0.2 \mathrm{~V}$. 
Table 6.3 Simulation results for a three-inverters chain driving a large load at $V_{\mathrm{DD}}=0.2 \mathrm{~V}$. In each intermediate node three minimum-size inverters are connected in parallel as offpath logic gates. 88 


\section{List of Symbols}

\begin{tabular}{|c|c|c|}
\hline Symbol & Definition & Unit \\
\hline$\emptyset_{s}$ & Surface Potential & $\mathrm{V}$ \\
\hline$C_{j 0}$ & Junction Capacitance at zero bias & $\mathrm{F}$ \\
\hline$\Psi_{b}$ & Built-in potential & $\mathrm{V}$ \\
\hline$\epsilon_{S i}$ & Permittivity of Silicon & $\mathrm{F} / \mathrm{m}$ \\
\hline$\epsilon_{o x}$ & Vacuum Permittivity & $\mathrm{F} / \mathrm{m}$ \\
\hline$\mu$ & Charge Mobility & $\mathrm{cm}^{2} /(\mathrm{V} . \mathrm{s})$ \\
\hline$\mu_{n}$ & Electron mobility & $\mathrm{cm}^{2} /(\mathrm{V} . \mathrm{s})$ \\
\hline$\mu_{p}$ & Hole Mobility & $\mathrm{cm}^{2} /(\mathrm{V} . \mathrm{s})$ \\
\hline C & Capacitance & $\mathrm{F}$ \\
\hline$C_{a v}$ & Average Capacitance & $\mathrm{F}$ \\
\hline$C_{\text {dep }}$ & Depletion Layer Capacitance per Unit Area & $\mathrm{F} / \mathrm{cm}^{2}$ \\
\hline$C_{\mathrm{GG}}$ & Total Gate Capacitance & $\mathrm{F}$ \\
\hline$C_{\mathrm{DB}}$ & Drain to Body Capacitance & $\mathrm{F}$ \\
\hline$C_{\mathrm{SB}}$ & Source to Body Capacitance & F \\
\hline$C_{\mathrm{j}}$ & Junction Capacitance per Unit Area & $\mathrm{F} / \mathrm{cm}^{2}$ \\
\hline$C_{\mathrm{jsw}}\left(C_{\mathrm{jswg}}\right)$ & Side-wall Junction Capacitance per Unit Length & $\mathrm{F} / \mathrm{cm}$ \\
\hline$C_{o x}$ & Field Oxide Capacitance & $\mathrm{F} / \mathrm{cm}^{2}$ \\
\hline$E_{D Y N}$ & Dynamic Energy Consumption & $\mathbf{J}$ \\
\hline$E_{S T}$ & Static (Leakage) Energy Consumption & $\mathrm{J}$ \\
\hline$E_{T}$ & Total Consumed Energy & $J$ \\
\hline$I_{d s}$ & Drain-Source Current & A \\
\hline$I_{d s a t}$ & Saturation current & A \\
\hline Igate & Gate Leakage Current & A \\
\hline Ijunct & Source and Drain Junctions Leakage Current & A \\
\hline Ileakage & Leakage Current & A \\
\hline$I_{\text {Nav }}$ & NMOS Transistor Average Current & A \\
\hline$I_{\text {off }}$ & Transistor "Off" Current & A \\
\hline
\end{tabular}




\begin{tabular}{|c|c|c|}
\hline$I_{\text {on }}$ & Transistor "On" Current & A \\
\hline Ion-av & Average ON current & A \\
\hline$I_{P a v}$ & PMOS Transistor Average Current & A \\
\hline$I_{s c-\mathrm{av}}$ & Average Short-Circuit Current & A \\
\hline$I_{\text {sub }}$ & Sub-threshold leakage current & A \\
\hline$L$ & Transistor Length & $\mathbf{m}$ \\
\hline$L_{\text {CoCmax }}$ & Channel Length Resulting in Maximum CoC & $\mathrm{m}$ \\
\hline$L_{D}$ & Lateral Diffusion & $\mathrm{m}$ \\
\hline$L_{D \min }$ & Channel Length Resulting in Minimum Delay & $\mathrm{m}$ \\
\hline$L_{E \min }$ & Channel Length Resulting in Minimum Energy & $\mathrm{m}$ \\
\hline$L_{f \max }$ & Channel Length Resulting in Maximum Frequency & $\mathrm{m}$ \\
\hline LImax & Channel Length Resulting in Maximum Current & $\mathrm{m}$ \\
\hline$M_{j}$ & Junction Grading Coefficient & \\
\hline$n$ & Sub-threshold Slope Factor & \\
\hline$N_{\text {sub }}$ & Substrate Doping & $\mathrm{cm}^{-3}$ \\
\hline$P_{\mathrm{C}}$ & Fitting Parameter in the Alpha Power law & \\
\hline$P_{D Y N}$ & Dynamic Power Consumption & W \\
\hline$P_{s c}$ & Short-Circuit Power Consumption & $\mathrm{W}$ \\
\hline$P_{S T}$ & Static Power Consumption & W \\
\hline$P_{s w}$ & Switching Power Consumption & $\mathrm{W}$ \\
\hline$P_{\mathrm{v}}$ & Fitting Parameter in the Alpha Power law & \\
\hline$Q_{d e p}$ & Depletion Charge per Unit Area & $\mathrm{C} / \mathrm{cm}^{2}$ \\
\hline$s$ & Sub-threshold Slope & $\mathrm{mV} / \mathrm{dec}$ \\
\hline$t_{o x}$ & Gate Oxide Thickness & $\mathrm{m}$ \\
\hline$t_{p}$ & Propagation Delay & $\mathbf{s}$ \\
\hline$t_{p h l}$ & High-to Low Propagation Delay & $\mathbf{s}$ \\
\hline$t_{p l h}$ & Low-to High Propagation Delay & $\mathbf{s}$ \\
\hline$V_{D D}$ & Supply Voltage & $\mathrm{v}$ \\
\hline$V_{d s}$ & Drain-Source Voltage & $\mathrm{v}$ \\
\hline$V_{d s a t}$ & Saturation Voltage & $\mathrm{v}$ \\
\hline
\end{tabular}




$\begin{array}{llc}V_{f b} & \text { Flat-band Voltage } & \mathrm{V} \\ V_{g s} & \text { Gate-Source Voltage } & \mathrm{V} \\ V_{R} & \text { Revers Bias On Junction } & \mathrm{V} \\ v_{\mathrm{T}} & \text { Thermal Voltage } & \mathrm{V} \\ V_{t h} & \text { Threshold Voltage } & \mathrm{V} \\ V_{t h n} & \text { NMOS Transistor Threshold Voltage } & \mathrm{V} \\ V_{t h p} & \text { PMOS Transistor Threshold Voltage } & \mathrm{V} \\ W & \text { Transistor Width } & \mathrm{m} \\ W_{d e p} & \text { Width of Depletion Region under the Gate } & \mathrm{m} \\ \alpha & \text { 1)Switching Activity Factor } & \\ & \text { 2) Velocity Saturation Index } & \mathrm{V} \\ \phi_{s t} & \text { Surface Potential at Threshold Condition } & \mathrm{A} / \mathrm{V}^{2} \\ \beta & \text { Transconductance } & \mathrm{V} \\ V_{\mathrm{IH}} & \text { Minimum input voltage to an inverter that is } & \\ & \text { considered a logic "1" } & \\ V_{\mathrm{IL}} & \text { Maximum input voltage to an inverter that is } & \mathrm{V} \\ & \text { considered a logic "0" } & \\ V_{\mathrm{OH}} & \text { Minimum Output Voltage of an Inverter that } & \mathrm{V} \\ & \text { Indicate a logic "1" } & \\ V_{\mathrm{OL}} & \begin{array}{l}\text { Maximum Output Voltage of an Inverter that } \\ \end{array} & \mathrm{V}\end{array}$




\section{List of Abbreviations}

\begin{tabular}{ll}
\hline Abbreviation & Definition \\
\hline CoC & Current-over-Capacitance \\
CPL & Complementary Pass-Transistor-Logic \\
DIBL & Drain Induced Barrier Lowering \\
DVL & Dual-Value-Logic \\
EDP & Energy Delay Product \\
GND & Ground \\
INWE & Inverse Narrow Width Effect \\
LOCOS & Local-Oxidation of Silicon \\
Ivt & Low-voltage-threshold \\
MOSFET & Metal-Oxide-Semiconductor Field Effect Transistor \\
NMOS & n-Channel MOSFET \\
NWE & Narrow Width Effect \\
PDP & Power Delay Product \\
PMOS & p-Channel MOSFET \\
PTL & Pass-Transistor-Logic \\
RSCE & Reverse Short Channel Effect \\
SCE & Short Channel Effect \\
SNM & Static Noise Margin \\
SRAM & Static Random-Access Memory \\
STI & Shallow Trench Isolation \\
svt & Standard-voltage-threshold \\
UDVS & Ultra-Dynamic-Voltage-Scaling \\
ULP & Ultra-Low-Power \\
VTC & Voltage Transfer Characteristics \\
\end{tabular}




\section{Introduction}

In the area of VLSI system design, considerable attention has been given to the design of high-performance processors and memories. However, in recent years, the demand for Ultra-Low-Power (ULP) applications has grown significantly. This tremendous demand has mainly been due to the fast growth of portable electronics market. Users are interested in having their portable devices operating for longer times per charge. Hence, in these applications, energy-efficiency is of primary importance.

Several approaches have been explored by researchers to reduce the power consumption in a digital circuit. The most straight forward method to reduce the power consumption is utilizing supply voltages lower than the nominal ones. According to [1], operating at supply voltages less than the threshold voltage of transistors, called subthreshold operation, reduces the energy consumption by an order of magnitude. In addition, the minimum energy operation, i.e., operating at a supply voltage where the energy consumption is at its lowest, also occurs in the sub-threshold region [1]. This, however, causes drastic performance degradation due to significant decrease in the driving current.

In some ULP applications such as biomedical devices, where operation frequencies are low and the main challenge is to keep the energy-consumption level as low as possible, sub-threshold design is the best solution. However, the challenge is to widen the span of the operation frequency of sub-threshold circuits to the level of mid-performance applications with no or minimal cost in energy.

\subsection{Motivation}

A number of projects have been performed to make the sub-threshold circuits more suitable for higher frequency applications. These projects spread from the transistor-level to system-level solutions [2] [3] [4] [5] [6] [7]. 
An effective way for enhancing the speed of sub-threshold circuits is to manipulate the dimensions of the transistors. Two of my teammates have chosen to study the effect of the transistor channel width on the speed of sub-threshold circuits. They came up with valuable results in their Master's theses [6] [7]. They proposed methods on finding optimum channel widths that improve the delay drastically. They also introduced a novel technique on using several parallel-transistors instead of a single wide transistor. They applied their methods to different types of circuits, from simple Ring Oscillators (RO), to a more complicated 32-bit Carry-Look-Ahead (CLA) adder, and the results were considerable.

However, I decided to look into the problem from another aspect. I decided to study the effect of the channel length on the sub-threshold circuits' behaviour. I obtained this idea when I was doing my first studies on the feasibility of Pass-Transistor-Logic (PTL) design in this mode of operation. I came across several papers that had the same idea but with a number of major drawbacks.

The first paper that suggests channel length manipulations in the sub-threshold design improvement is [8]. In this paper authors have utilized Reverse-Short-ChannelEffects (RSCE) in their designs. This work suffers from a number of shortcomings. First, their PMOS transistor is apparently improperly biased. I biased a PMOS transistor in TSMC $130 \mathrm{~nm}$ technology kit and plotted the current versus the channel length through simulation in Cadence CAD tool. I found that if we bias the PMOS transistor in a wrong way, the plot will be the same as the current plot presented in the paper. This mistake resulted in using a non-optimal channel length for PMOS transistors. Second, the derivative obtained for the current with respect to the channel length is mathematically incorrect. I found this mistake by calculating the derivative by hand. In addition this result is not carried on in designing discussed circuits in the paper. Third, although it is known that minimum-sized sub-threshold circuits perform competitively well, no comparison is reported between their proposed circuits and minimum-size transistor circuits. Fourth, the authors applied their findings to some ISCAS test benches and showed that the performance and power consumption improved. ISCAS test benches use transistor sizing that are more suitable for super-threshold operation, where the InverseNarrow-Width Effect (INWE) and RSCE are not observed. 
The second paper that uses longer channel length in the sub-threshold region is [9]. In this work, the channel length is manipulated to achieve the minimum-energy consumption for power supplies between 300 to $700 \mathrm{mV}$ that cannot be usually considered as sub-threshold operation modes. This work does not address any speed improvements and comparisons to circuits with minimum-size transistors.

There are several other papers that study the channel length effect on the performance and energy improvement in the sub-threshold region, such as [2] [10] [11]. In none of them a methodology to find the optimum channel length minimizing the delay is presented.

Accordingly, I decided to study the effect of channel length manipulation on the behaviour of a transistor in the sub-threshold region, and utilize the results in designing digital circuits block. In this work, my main research focus is on the frequency and delay optimizations. However, in some cases where there is a need to discuss the energy consumption, it is addressed as well.

\subsection{Thesis Objective}

This thesis follows the objectives listed below.

- To study the behaviour of a MOSFET in the sub-threshold region with respect to its dimensions.

- To perform a comparative study of the effects of the channel width and channel length manipulation on important parameters of MOSFETs such as the threshold voltage, current, capacitances, sub-threshold slope, and oncurrent to off-current ratio $\left(I_{\text {on }} / I_{\text {off }}\right)$.

- To exploit the sub-threshold behaviour of transistors to find the optimum channel length for minimizing the delay.

- To propose a design methodology for delay optimization for sub-threshold circuits through transistors' channel length manipulation.

- To apply the methodology to design some common circuits and verify its effectiveness. 


\subsection{Thesis Organization}

After this introductory chapter, the second chapter presents a historical background of the origin of ULP applications, challenges, and the offered solutions for them. Subthreshold operation is introduced as the best candidate to solve the problems of many ULP circuits. Challenges in designing a sub-threshold circuit are addressed and the potentials of different research areas are presented.

The third chapter covers the background information that is needed in studying the transistor behaviour study and digital circuits figure of merits.

In Chapter 4, we present a detailed study on transistor's important parameters with respect to its dimensions, such as the threshold voltage, current, capacitances, and subthreshold slope.

In Chapter 5, based on the current and capacitance behaviour presented in Chapter 4, the Current-over-Capacitance $(\mathrm{CoC})$ method is presented. First, the $\mathrm{CoC}$ method is developed for one transistor to find its optimum channel length. Then, this method is extended for serial and parallel combinations. The effectiveness of the optimum channel lengths is verified by incorporating them in different ROs.

In Chapter 6, some applications of our proposed methodology are presented. The obtained optimum channel lengths in Chapter 5 are used in a 32-bit CLA adder and a chain of inverters driving a large load. Improvements in both the delay and energy are reported.

Finally, Chapter 7 presents the concluding remarks. 


\section{Literature Review: ULP and Sub-threshold Circuits}

When the first integrated electronic circuit built on a single slice of Germanium by Jack Kilby in 1958 at Texas Instruments in Dallas, nobody knew that this invention will revolutionize electronics market, and consequently the life of everyday [12]. Now, several decades later, his invention known as Integrated Circuits (ICs), is being used in a wide range of applications, from high-technology space crafts to small toys for kids.

The ICs world is accustomed at this point to following the Moore's Law. In 1965, Gordon Moore observed that the number of transistors that can be most economically manufactured on a chip doubles every 18 months [13]. Since that time, the IC industry has maintained the astonishing exponential trend, which Moore first observed, by continuing to scale down the size of transistors to have faster, cheaper, and less power consuming ICs (i.e., Dennard's Scaling Law) [14].

The traditional goal has been to reduce the minimum feature size by $30 \%$ with each new technology. This scaling, theoretically, results in $30 \%$ and $50 \%$ reduction in logic gate's delay and chip area, respectively. Likewise, active power should decrease for a given circuit due to smaller transistors and lower supply voltages [3]. Obviously, this scaling cannot go on forever because transistors cannot be smaller than atoms. Dennard Scaling Law has already begun to slow. In 1990s, experts agreed that the scaling would continue for at least a decade, but in 2009, they predicted that Moore's Law will continue for another decade [15].

Despite the reduction of power consumption in each individual transistor caused by scaling, the total power consumption per chip has drastically increased due to the exponential growth in the number of integrated devices per chip and the increase in the clock frequency. A power consumption of $\mathbf{8 ~ W}$ is reported for an Intel Pentium CPU operating at $75 \mathrm{MHz}$, whilst the power consumption is increased to $150 \mathrm{~W}$ for a newer CPU generation, Core 2 Extreme QX9775 operating at $3.2 \mathrm{GHz}$ [16]. This increase in 
the power consumption that is noticeable in high-performance applications such as microprocessors, introduces new challenges to both fabrication and circuit design engineers, like the need for special packaging for the quick removal of the produced heat inside the chip and designing more stable circuits with respect to temperature variations inside the chip. Therefore, exploring design methodologies for low-power circuit is of great importance.

In addition to the heat problem, demands for portable battery-operated devices have increased significantly over the last decades. Taking a laptop as an example, consumers are strongly calling for laptops with lower price but much longer running time per charge. Furthermore, in some applications like implantable biomedical devices, where changing the battery needs a surgery, a small battery should work for tens of years inside the patient's body. Also, while the number of transistors integrated on a chip doubles every 18 months based on the Moore's Law, the capacity density of batteries doubles only every 10 years [17]. Hence, the energy consumption becomes a bottleneck rather than the performance for many applications.

All of this attention to the power and energy consumption in circuit design has created a significant research potential for minimizing or at least reducing the energy or power consumption. In the following, first, a brief history of ULP applications is presented. Then, the sub-threshold operation is introduced as a solution for most ULP applications. The challenges in the design of sub-threshold circuits are discussed next. Thereafter, the previous related sub-threshold work is reported to address how researchers have faced these challenges and what are the drawbacks of their researches.

\subsection{ULP Applications}

The first ULP application goes back to 60's when the Centre Electronique Horloger Neuchatel developed an electronic wristwatch that consuming less than $30 \mu \mathrm{A}$ from a $1.3 \mathrm{~V}$ supply voltage [18]. This was the only ULP application for 30 years, until a couple of decades ago that the new class of ULP applications were introduced. ULP applications are categorized into high performance and low to medium performance. In the first 
group, performance and power (energy) consumption have the same level of importance.

Table 2.1 summarizes digital circuit techniques in these kinds of applications.

Table 2.1 Low-power techniques for high-performance digital circuits [19].

\begin{tabular}{|c|c|}
\hline$\overline{\text { Design hierarchy }}$ & Reported low-power digital techniques \\
\hline Algorithmic level & $\begin{array}{l}\text { 1- Using more efficient DSP algorithms to } \\
\text { eliminate unnecessary computation and reduce } \\
\text { the number of computation }\end{array}$ \\
\hline Mapping and architecture level & $\begin{array}{l}\text { 1- ISA extension } \\
\text { 2- Scenario based mapping, rescheduling, etc. } \\
\text { 3- Preserving data correlation and reference } \\
\text { locality, reducing memory access } \\
\text { 4- Common expression elimination, pre- } \\
\text { computation, etc. } \\
\text { 5- Using suitable pipelining and parallelism, } \\
\text { enabling low supply voltage/frequency }\end{array}$ \\
\hline System level & $\begin{array}{l}\text { 1- Multiple supply voltage (MVS) } \\
\text { 2- Dynamic voltage scaling (DVS) } \\
\text { 3- Dynamic voltage-frequency scaling (DVFS) } \\
\text { 4- Multiple clock domains } \\
\text { 5- Dynamic- Variable threshold voltage (adaptive } \\
\text { body biasing) } \\
\text { 6- Sleep and power down modes }\end{array}$ \\
\hline Circuit level & $\begin{array}{l}\text { 1- Power gating, clock gating } \\
\text { 2- Logic sizing and re-structuring } \\
\text { 3- Adiabatic logic circuits } \\
\text { 4- Low power SRAM and DRAM } \\
\text { 5- Power efficient DC-DC level converter }\end{array}$ \\
\hline Device level & $\begin{array}{l}\text { 1- Multiple threshold CMOS } \\
\text { 2- Low temperature CMOS } \\
\text { 3- Silicon-on-Insulator } \\
\text { 4- Low power packaging }\end{array}$ \\
\hline
\end{tabular}


In the second group of ULP applications power and more specifically energy is a fundamental constraint. These ULP applications do not require the ultimate performance of high-end processors. Among them, Radio-Frequency Identifier (RFID) tags and Micro-sensor networks are attracting more attentions. RFID tags are used to wirelessly identify an object, an animal or a person [20]. Micro-sensor networks refer to the physical hardware that provides sensing, processing, and wireless communication. These networks consist of thousands of distributed nodes that sense and process data and send it to the end-user. Micro-sensors are used in habitat monitoring [21], environment observation and forecasting systems [22], structural monitoring [23], and health monitoring [24] [25]. Other examples for micro-sensor networks include automotive industries, military applications for early biological and chemical weapons detection, and traffic density monitoring in cities. Table 2.2 lists some micro-sensor networks applications. For each application, the associated sampling rates (in $\mathrm{Hz}$ ) and the sample precision (in bits per sample) are also indicated.

Table 2.2 Micro-sensor networks applications [17]

\begin{tabular}{|c|c|c|c|}
\hline \multicolumn{2}{|c|}{ Application } & Sample Rate (in Hz) & Sample Precision (in bits) \\
\hline $\begin{array}{l}\text { Biomedical } \\
\text { Applications }\end{array}$ & $\begin{array}{c}\text { Body temperature } \\
\text { Heart Rate } \\
\text { Blood Pressure } \\
\text { Electro-Enccephlo- } \\
\text { Graph (EEG) } \\
\text { Electro-Cardio-Graph } \\
\text { (ECG) } \\
\text { Electro-Myo-Graph } \\
\text { (EMG) } \\
\text { Hearing Aids }\end{array}$ & $\begin{array}{c}0.1 \sim 1 \\
0.8 \sim 3.2 \\
50 \sim 100 \\
100 \sim 200 \\
100 \sim 250 \\
100 \sim 5000 \\
15000 \sim 44000\end{array}$ & $\begin{array}{l}8 \\
1 \\
8 \\
16 \\
8 \\
8 \\
16\end{array}$ \\
\hline $\begin{array}{c}\text { Climate } \\
\text { Monitoring }\end{array}$ & $\begin{array}{l}\text { Ambient light level } \\
\text { Ambient noise level } \\
\text { Barometric pressure } \\
\text { Wind direction }\end{array}$ & $\begin{array}{l}0.017 \sim 1 \\
0.017 \sim 1 \\
0.017 \sim 1 \\
0.2 \sim 100\end{array}$ & $\begin{array}{l}16 \\
16 \\
8 \\
8\end{array}$ \\
\hline $\begin{array}{c}\text { Automotive } \\
\text { Industry }\end{array}$ & $\begin{array}{c}\text { Engine temperature } \\
\text { Tire pressure }\end{array}$ & $\begin{array}{l}100 \sim 150 \\
100 \sim 150\end{array}$ & $\begin{array}{l}16 \\
16\end{array}$ \\
\hline
\end{tabular}


The low-power techniques listed in Table 2.1 are not effective enough for the applications listed in Table 2.2. These entire applications share a common characteristic: their low computational load, and a common constraint: tiny power or energy consumption. Indeed, these applications either have to operate for a long time on small batteries or harvest energy from the ambient.

In some micro-sensor networks applications, especially biomedical applications, where the micro-sensor should be implanted inside the patient's organ, the battery have to be very small and long-lasting. As a reference, a $1 \mathrm{~cm}^{3}$ Lithium battery has $1.5 \mathrm{KJ}$ of capacity, which means it can deliver $10 \mu \mathrm{W}$ of power over five years [26]. In some other kinds of micro-sensor networks, they gain their energy by harvesting it from the ambient. Table 2.3 lists the typical power harvesting mechanisms and their typical delivered power densities. The power available from these sources depends on the area of the power source and environmental conditions at any given time. It is reasonable to expect tens of $\mu \mathrm{W}$ to be harvested from the ambient energy. Therefore, according to the capacity of the batteries and power harvesting mechanisms, micro-sensor nodes must keep their average power consumption in the $10-100 \mu \mathrm{W}$ range [27].

Table 2.3 Example of power harvesting mechanism and their typical power densities.

\begin{tabular}{cc}
\hline \hline Mechanism & Power Density $\left(\boldsymbol{\mu W} / \mathrm{cm}^{2}\right)$ \\
\hline Electromagnetic Vibration [28] & 4 \\
Piezoelectric Vibration [29] & 500 \\
Electrostatic Vibration [30] & 3.8 \\
Thermoelectric [31] & 60 \\
Solar-Direct sunlight [32] & 3700 \\
Solar-Indoor [32] & 3.2 \\
\hline
\end{tabular}

Notice that, the important figures of merit to consider are different, depending on the energy or power source. In battery-operated systems, the energy to perform an operation has to be low enough to have a reasonable battery life span. While, for environment energy harvesting systems, where the available energy that can be harvested is limited but 
ample time is available, the power has to be minimized. Because the energy source remains available, the fact that an operation takes more energy is less important than keeping the circuit power below the available constraint.

\subsection{Why Sub-threshold?}

As presented in the previous section, there are many ULP applications that need small battery size and small amount of produced heat, especially in biomedical applications to prevent any damage to the tissues [24]. It seems that lowering the supply voltage is the best solution to meet the requirements of such applications. Both energy and power consumption depend on the supply voltage, i.e., lowering the power supply will decrease both. Now, there is a question to be answered. What is the minimum limit of the power supply? Can we reduce the supply voltage indefinitely?

Swanson and Meindl studied the limits of voltage scaling in the early 1970s [33]. They showed in theory that an inverter could maintain its functionality till $V_{\mathrm{DD}}=4 v_{\mathrm{T}}$, where $V_{\mathrm{DD}}$ is the supply voltage and $v_{\mathrm{T}}$ is the thermal voltage, which is $26 \mathrm{mV}$ at room temperature. Soon after, they implemented a RO and found $100 \mathrm{mV}$ as the minimum practical limit for the supply voltage [34]. In [35], the authors claimed a minimum operational voltage of $48 \mathrm{mv}$, theoretically, and verified it by SPICE simulations in the $0.18 \mu \mathrm{m}$ technology.

Operating in this new range of supply voltage, which is less than transistors' threshold voltage, is referred as Sub-threshold operation. This mode of operation involves using a supply voltage in the 0.2 to $0.4 \mathrm{~V}$ range that is substantially lower than the nominal supply voltage (which fall to in the 0.9 to $1.2 \mathrm{~V}$ range) for the modern CMOS technologies.

Decreasing the power supply reduces both the active and static consumed power by a circuit. The static or leakage power $\left(P_{\mathrm{ST}}\right)$ in a circuit is given by

$$
P_{\mathrm{ST}}=V_{\mathrm{DD}} \times I_{\text {Leak }}
$$

where $I_{\text {Leak }}$ is the leakage current. Lowering the supply voltage is the most straightforward way of reducing the leakage power consumed by the circuit. For 
example, if we reduce $V_{\mathrm{DD}}$ from its nominal value in the $130 \mathrm{~nm}$ technology, $V_{\mathrm{DD}}=1.2 \mathrm{~V}$, to a $V_{\mathrm{DD}}$ in the sub-threshold region, e.g., $V_{\mathrm{DD}}=0.2 \mathrm{~V}, P_{\mathrm{ST}}$ decreases six times, providing that the leakage current is constant. Table 2.4 shows a saving in $P_{\mathrm{ST}}$ by $2.25-6$ times, only from the supply voltage reduction assuming a fixed leakage current.

Table 2.4 Leakage power savings from voltage reduction (constant leakage current).

\begin{tabular}{|c|c|c|c|c|}
\hline & \multicolumn{4}{|c|}{ Nominal $V_{\text {DD }}(\mathrm{V})$} \\
\hline Sub-threshold $\boldsymbol{V}_{\mathrm{DD}}(\mathrm{V})$ & $\mathbf{0 . 9}$ & $\mathbf{1}$ & $\mathbf{1 . 1}$ & $\mathbf{1 . 2}$ \\
\hline $\mathbf{0 . 4}$ & $2.25 \mathrm{X}$ & $2.5 \mathrm{X}$ & $2.75 \mathrm{X}$ & $3 \mathrm{X}$ \\
0.3 & $3 \mathrm{X}$ & $3.3 \mathrm{X}$ & $3.7 \mathrm{X}$ & $4 \mathrm{X}$ \\
0.2 & $4.5 \mathrm{X}$ & $5 \mathrm{X}$ & $5.5 \mathrm{X}$ & $6 \mathrm{X}$ \\
\hline
\end{tabular}

Since lowering the supply voltage also decreases the leakage current, in the real case, savings in the static power by reducing $V_{\mathrm{DD}}$ are even more than those reported in Table 2.4. Figure 2.1 shows a five times reduction in the leakage current when the supply voltage scales down from $1.1 \mathrm{~V}$ to $0.2 \mathrm{~V}$. Combining the changes in the leakage current with the results of Table 2.4 leads to a saving of 6.5 to 30 times in $P_{\mathrm{ST}}$.

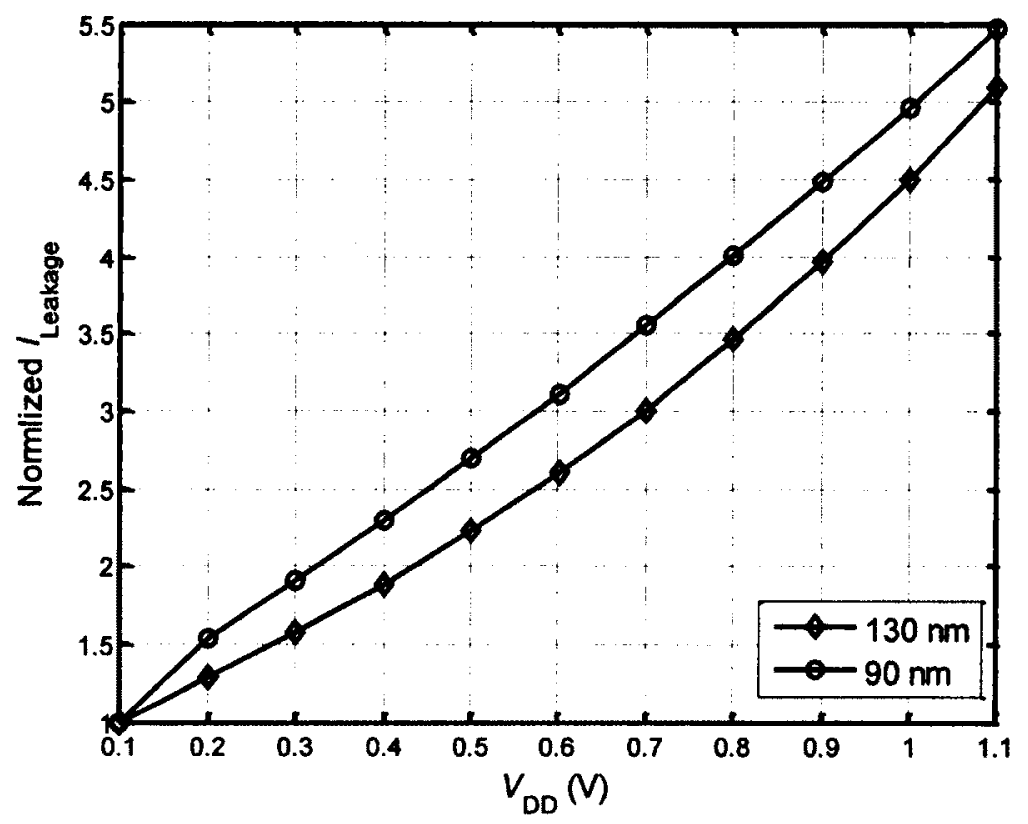

Figure 2.1 Normalized static current for an inverter versus $V_{D D}$ in $90 \mathrm{~nm}$ and $130 \mathrm{~nm}$ technologies at minimum sizes. 
One of the side effects of operating in the sub-threshold region is a significant increase in the delay, due to the very small on-current. Delay in sub-threshold circuits is orders of magnitude more than that of super-threshold circuits. However, they are still useful in many ULP applications with low speed, as described in Section 2.1.

Due to the significant increase in the delay for circuits operating in the sub-threshold region, the static or leakage energy $\left(E_{\mathrm{ST}}\right)$ increases as the supply voltage reduces. The Static energy depends on how fast an operation takes place. In spite of the static power reduction by lowering the supply voltage, the static energy increases (Figure 2.2 (a)). On the other hand, since the dynamic energy is proportional to $V_{\mathrm{DD}}{ }^{2}$, it decreases quadratically by lowering $V_{\mathrm{DD}}$ (Figure 2.2 (a)). The total energy, which is the sum of the static and dynamic energies, decreases until the static energy becomes dominant and creates a minimum point, as illustrated in Figure 2.2(b). This minimum-energy point consistently occurs at the sub-threshold region [1] [36] [37].

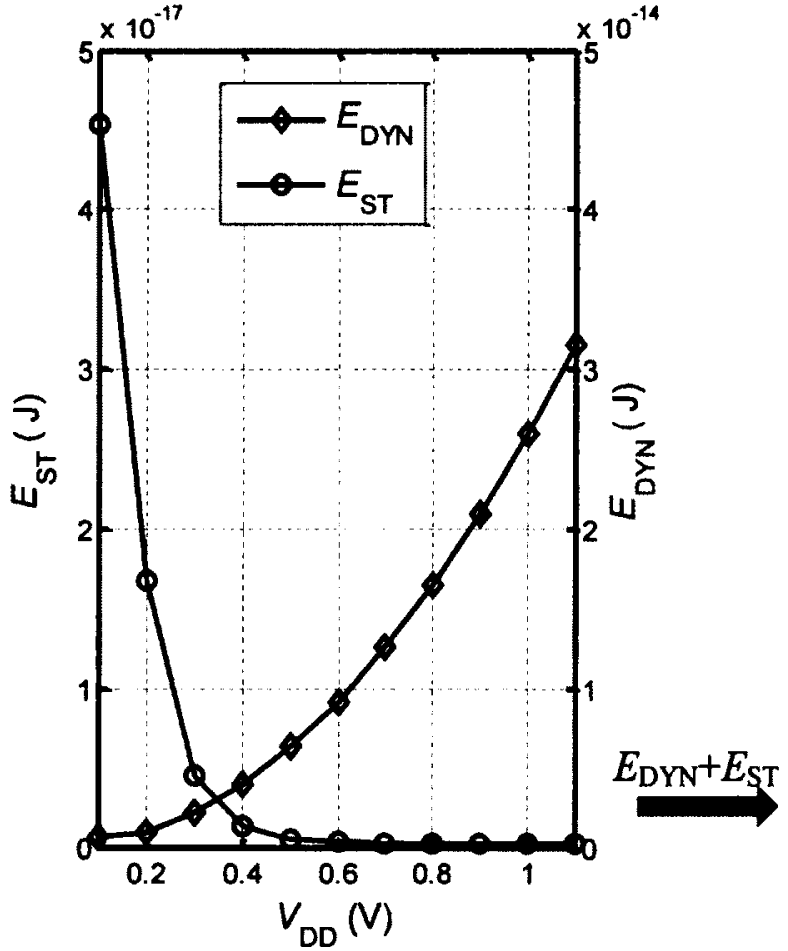

(a)

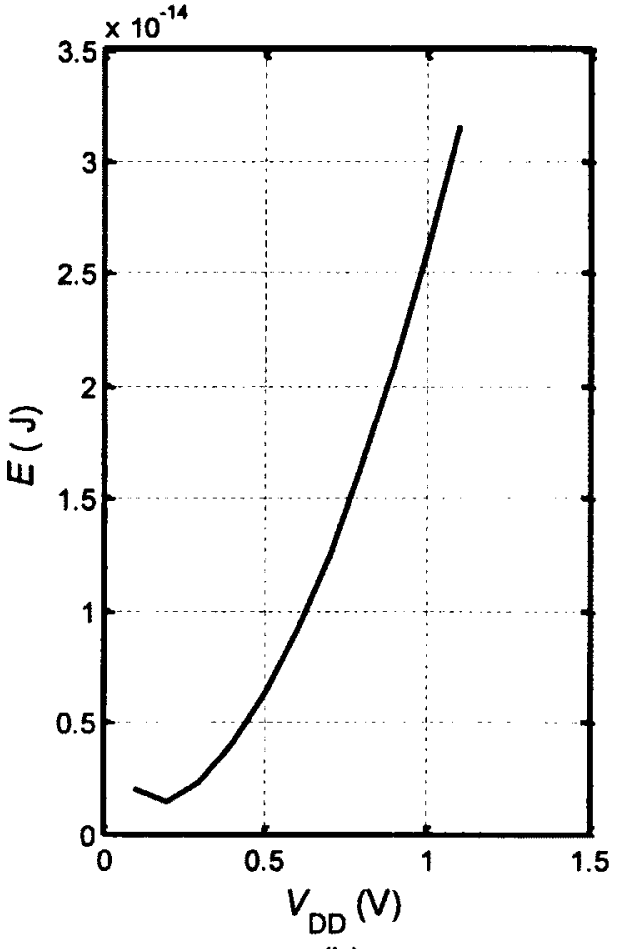

(b)

Figure 2.2 Static and Dynamic energy (a) and total energy (b) versus $V_{\text {DD }}$ for 29-inverter $\mathrm{RO}$ in $130 \mathrm{~nm}$ technology at minimum size. 
When Vittoz and Fellrath demonstrated the first analog circuit operating in the subthreshold region at the 1976 European Solid-State Circuits Conference (ESSCIRC) [38], the audience suggested that such circuits could not be reliable, as they operate with the leakage current [39]. Thanks to the wrist-watch application, analog sub-threshold circuits received more attention than digital sub-threshold circuits until the 1999 IEEE/ACM International Symposium on Low-Power-Electronics and Design (ISLPED), where Soelemen and Roy showed that operation of CMOS and pseudo-NMOS logic gates down to $0.3 \mathrm{~V}$ leads to nearly two orders of magnitude power-delay product (PDP) saving in a $0.35 \mu \mathrm{m}$ technology [40].

After [1] and [40], despite the all challenges -to be introduced later- in the subthreshold circuits design, ULP sub-threshold circuits came out of the shadow and turned into a vibrant research area in digital electronics. Till 2008, a decade after Soeleman's first paper in sub-threshold logic, there had been numerous successful sub-threshold circuits implementations. Among the most advanced ones was a complete sub-threshold microcontroller with an embedded SRAM and a DC-DC converter in a $65 \mathrm{~nm}$ technology for biomedical applications designed in collaboration between MIT and Texas Instrument [4].

Although sub-threshold circuits originated for low-performance ULP applications, it does not mean that they are not applicable in high-performance circuits. When highperformance systems enter into the sleep mode to reduce the power consumption, some circuits are still operating to monitor the status of the whole system to decide the wake up time. One existing example is a standby leakage-reduction system that uses a feedback loop and Canary SRAM cells to the set standby $V_{\mathrm{DD}}$ to minimize the leakage while protecting the data in the SRAM [41]. Sub-threshold operation seems ideal for the sleepmode circuits.

Additionally, many high-performance systems, such as microprocessors used in laptops and smart phones, spend a large fraction of time with a smaller operational load than their maximum operational load. Sub-threshold operation can be used during these periods for the background computation that do not require high throughputs. This can reduce on-die temperature and energy cost. One practical implemented circuit is a 
combination of multi- $V_{\mathrm{DD}}$ with a small set of power switches to perform a flexible dynamic voltage scaling [42] [43].

Finally, the growing potential of large-scale microprocessors combined with their power constraints, which is becoming more prominent for these systems than before, (as their operation frequency grows rapidly) has opened a new research path for the subthreshold and near-threshold computing in parallel architectures such as many-core microprocessors [44].

\subsection{Roadmap of Sub-threshold Circuits Design}

Sub-threshold operation seems the best match for ULP applications as their usage are daily increasing. However, there are some challenges that sub-threshold designs face. Sub-threshold researches are focused on overcoming these challenges. These challenges are discussed in the following.

1- When the supply voltage drops below the threshold voltage, the transistor current still remains above zero. A nonzero gate-to-source $\left(V_{G S}\right)$ voltage that is less than transistor threshold voltage still produces a current that is larger than the offcurrent, $I_{\text {off }}$ (i.e., the transistor current when $V_{\mathrm{GS}}=0 \mathrm{~V}$ ). This finite ratio of the oncurrent, $I_{\text {on }}$, to $I_{\text {off }}$ lets sub-threshold digital gates behave statically in a similar fashion to the super-threshold gates. However, their transient behaviour is much slower than the super-threshold ones, due to the small drive currents. The frequency of operation in the sub-threshold region is orders of magnitude smaller than that of the super-threshold region. For instance, Figure 2.3 (top) shows that reducing the power supply from its nominal value, $1.1 \mathrm{~V}$, to the sub-threshold value, $0.2 \mathrm{~V}$, reduces the operating frequency by three orders of magnitude for about 20 times reduction in the total energy per operation.

2- As shown in Figure 2.3 (bottom), the $I_{\text {on }} / I_{\text {off }}$ ratio reduces about three orders of magnitude when the supply voltage reduces from its nominal value to the subthreshold value. This reduction in the $I_{\text {on }} / I_{\text {off }}$ ratio can lead to reliability problems. For example, for certain gates with parallel leaking path (e.g., NOR gates) or in keepers, degraded $I_{\mathrm{on}} / I_{\mathrm{off}}$ ratio results in a bad functionality. 
3- Process-Voltage-Temperature (PVT) variations that become more prominent in modern CMOS technologies, affect the transistor threshold voltage and consequently the current, both in the super-threshold and sub-threshold regions. However, this effect is more obvious in the sub-threshold region because of the exponential relation between the current and threshold voltage (i.e., $I_{D} \propto$ $\exp \left(V_{G S}-V_{t h}\right)$, where $V_{\text {th }}$ is the threshold voltage of transistor).

Despite the mentioned challenges, researchers have successfully developed techniques to build relatively fast and robust sub-threshold digital circuits ranging from small gates and SRAMs to processors. Overcoming these challenges needs a wide collaborative research at every design hierarchy level. Some of the important research topics are listed below.

- Device Optimization for Sub-threshold Operation: Standard transistors are super-threshold transistors and optimized for operation in their nominal
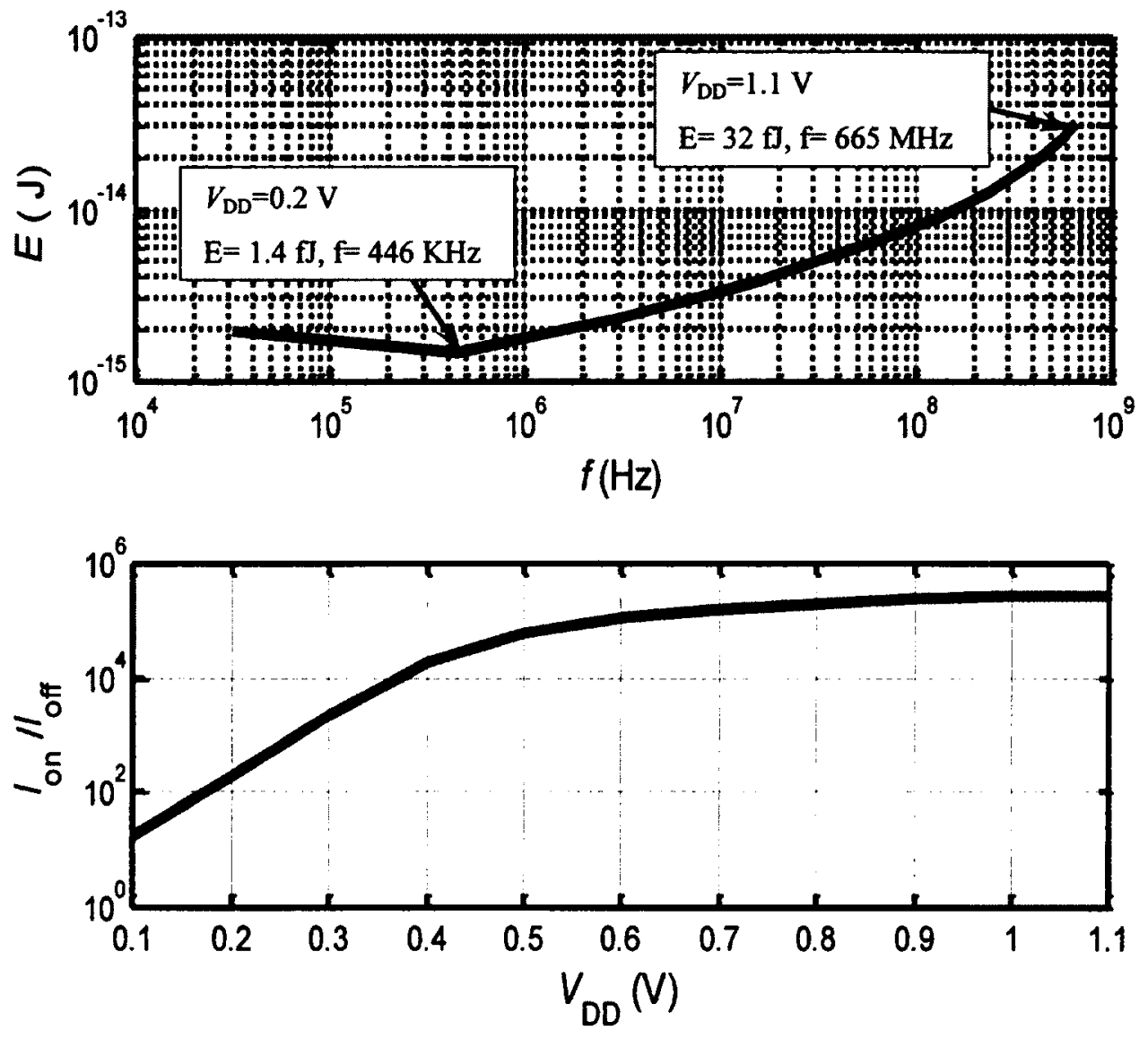

Figure 2.3 Energy vs. Frequency (top) and $I_{\text {on }} / I_{\text {off }}$ ratio vs. $V_{D D}$ (bottom) simulated in $130 \mathrm{~nm}$ technology. 
supply voltages for high-performance applications. It is an established fact that the doping profile and level in conventional transistors are designed to mitigate the Short-Channel-Effects (SCE), e.g., punch-through. However, In the sub-threshold region the supply voltage is small and SCEs are not significant. It means that many of the process steps to produce special drain and source region or channel doping tuning are not any more necessary for transistors operating in the sub-threshold region [45]. On the other hand, the gate oxide thickness should be optimized for this region of operation. Reducing the oxide thickness does not always impose a positive effect in subthreshold operation. Reducing the oxide thickness decreases the sub-threshold slope [46], which is desirable for this mode of operation, and in parallel, increases the gate capacitance that causes more delay and energy consumption. Hence, the oxide thickness should be optimized for subthreshold operation. Also, a new transistor structure, called the Double Gate MOSFET, is introduced for sub-threshold operation, that shows a more ideal sub-threshold slope. In this kind of transistors a longer channel length can be used to have more robust ULP circuits [3] [46] [47].

- Transistor Modelling: Unlike the super-threshold, where good and valid models are established for current and capacitances, in the sub-threshold region there is a need to model transistors. Many of researchers are working on this topic [48] [49] [50] [51].

- Transistor Sizing: There is a great need to speed up sub-threshold circuits to expand ULP circuits applications to higher frequencies. A conventional method to increase the speed of a circuit in the super-threshold region is, increasing manipulating the channel width of the transistors through applying the method of logical effort. Such methods are very powerful as long as digital circuits operate in the super-threshold region. However, applying logical effort for sub-threshold operation is quite different, because the current may not show a linear relation with the channel width [52] [53] [54] [55] [56]. On the other hand, in most super-threshold circuits the channel length is set to its minimum value. But, it has been shown that increasing the 
channel length to few folds of its minimum value improves the performance of circuits operating in the sub-threshold region [2] [8] [9] [10] [11].

- Logic Families and Circuit Styles: Some of circuit topologies that operate well under the super-threshold conditions might not be suitable for operating in the sub-threshold region, and vice versa. For example, pass-transistor-logic (PTL) families suffer from a threshold voltage drop in the super-threshold operation. To overcome this issue, keepers or transmission gates are introduced that increase the number of transistors and, as a result, the power consumption. Whilst, in the sub-threshold region there is no threshold voltage drop [57]. Reduced $I_{\text {on }} / I_{\text {off }}$ ratio in the sub-threshold region forces the designer to design more robust circuits. Some introduced techniques cannot be used in the super-threshold. For instance, Dynamic- $V_{\text {th }}-C M O S$ (DVTCMOS) uses transistors with gate and body tied together. DVTCMOS has the same characteristics as a conventional MOSFET in the "off" state. But, in the "on" state the threshold voltage reduces producing a larger on-current. This improves the $I_{\text {on }} / I_{\text {off }}$ ratio [58]. Sub-threshold logic circuits are slow and make these circuits more suitable for adiabatic computation [59]. Many studies have been performed on XOR gates, adder circuits, and some fundamental blocks use in sub-threshold digital circuits [60] [61] [62] [63].

- Energy Minimization: While energy minimization is not of primary importance for high-performance systems operating in the super-threshold region, it is a major topic in the sub-threshold design. Sub-threshold design has been introduced to meet the energy constraints in ULP applications. Hence, the designer in this area should be aware of the effect of different variables like $V_{\mathrm{DD}}, V_{\text {th }}$, and transistor sizes. In [1] [9] [36] [64] [65] [66] [67] [68] [69] analytical models are introduced to find the optimal $V_{\mathrm{DD}}, V_{\mathrm{th}}$, and transistor sizes to obtain the minimum energy consumption.

- SRAM Design: Energy-efficient sub-threshold design cannot succeed without robust and dense SRAMs. SRAM is an important component of many ICs, and it can contribute a large fraction of the active and static energy. The widely used 6T SRAM cell fails to operate in sub-threshold [70]. Reduced 
$I_{\text {on }} / I_{\text {off }}$ ratio complicates the reading and writing steps in the sub-threshold region. So, it is important to have SRAMs compatible with the sub-threshold systems. Some samples of research on sub-threshold SRAMs are presented in [10] [11] [71] [72].

- Architecture and System Level Design: Since an entire system may not be able to operate completely in the sub-threshold region, there is a need for periodic switching between the nominal supply voltage and sub-threshold supply voltage [35] [42] [37]. Also, connecting different parts of a system operating in different voltages needs DC/DC level converters [4]. In addition, pipelining and parallel architectures can increase the speed of sub-threshold circuits [43] [44].

Table 2.5 lists a summary of sub-threshold research topics. Although, no commercial applications have yet adapted this approach, it is expected that sub-threshold and nearthreshold circuits will make their way into commercial products.

Table 2.5 Summary of Sub-threshold papers

\begin{tabular}{|c|c|}
\hline Category & Existing Reference \\
\hline \hline Device Optimization & {$[2][45][46][47]$} \\
\hline Transistor Modeling & {$[48][49][50][51]$} \\
\hline Transistor Sizing & {$[8][52][53][54][55][56]$} \\
\hline Logic Style & {$[57]$} \\
\hline Cell Library & {$[73][74]$} \\
\hline Circuit Level & {$[5][38][40][58][59][60][61][62][63]$} \\
\hline Energy Minimization & {$[1][9][36][64][65][66][67][67][68][69]$} \\
\hline SRAM & {$[10][11][41][70][71]$} \\
\hline System Level Solution & {$[27][35][42][37]$} \\
\hline Architecture Level & {$[43][44]$} \\
\hline Processors & {$[1][4][72]$} \\
\hline Applications & {$[21][23][24][25]$} \\
\hline
\end{tabular}




\subsection{Chapter Summary}

The trend of electronics market shows an increasing tendency toward portable devices with light batteries but long life. To increase the span of batteries life time, ULP techniques have been developed since a couple of decades ago. Decreasing the supply voltage, which is the simplest approach, has introduced the sub-threshold region of operation as a strong candidate for ULP circuits. In this region, circuits consume the minimum energy, however, face several challenges. Among the challenges, the low speed is the most important one. Many research projects are being carried out to increase the speed of sub-threshold circuits in order to extend their application domain to higher frequency devices.

In the following chapter we will review the basic characteristics of a MOSFET and digital circuits. 


\section{Background}

In this introductory chapter, a brief description of MOSFET properties is given to serve as a background for the subsequent chapters. Also, some of important metrics and features of a CMOS digital circuit, such as propagation delay, power and energy consumption, are presented at the end of this chapter. Studying this brief introduction makes it easier to understand the effect of transistor sizing on the delay optimizing and the energy consumption, to be discussed in later chapters.

\subsection{MOSFET}

Figure 3.1 shows the structure of an n-channel MOSFET transistor (NMOS). The MOSFET is a four-terminal device. It is usually a symmetric device in which there is no physical difference between the source and drain terminals. The diodes created between the source and drain junctions and the substrate must be reversed-biased for the normal operation of the MOSFET. It means that in an NMOS the substrate is connected to most negative voltage (i.e., GND) and in a PMOS the substrate is connected to the most positive potential (i.e., $\left.V_{\mathrm{DD}}\right)$ in the circuit. The channel width $(W)$ and channel length $(L)$ have important effect on the transistor current. Also, these dimensions have direct effects on MOSFET capacitances and, consequently, on the propagation delay and energy consumption (Sections 3.2.1-3.2.3).

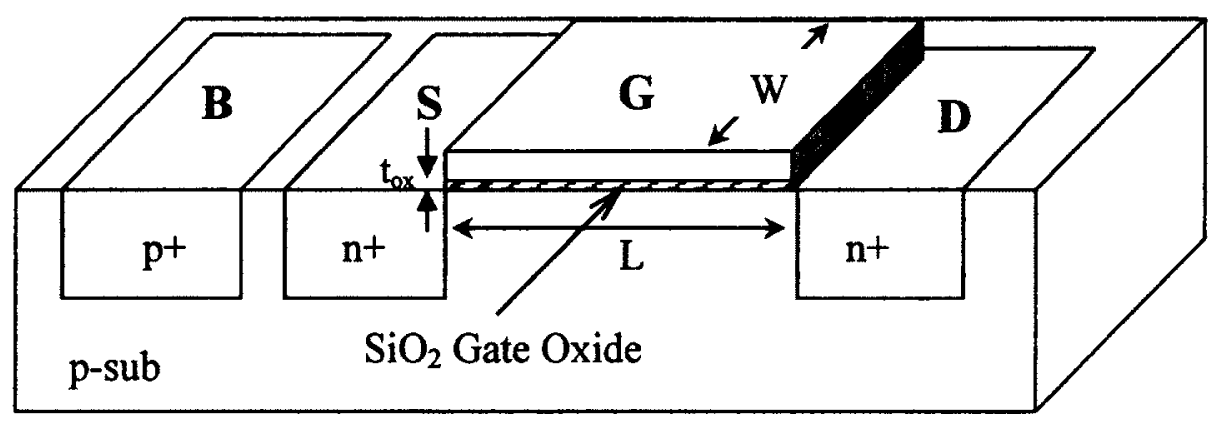

Figure 3.1 structure of an n-channel MOSFET (NMOS). 


\subsubsection{Current}

The most well-known MOSFET current model is Shockley model [75] that is valid only for long-channel transistors. Typically, when the dimensions of transistors shrink to submicron, some small-geometry effects like velocity saturation become prominent and this simple model is not valid any more. A simple short-channel model is proposed as the alpha-power law model by T. Sakurai et al [76] as

$$
I_{d s}=\left\{\begin{array}{cl}
0 & V_{g s}<V_{\text {th }} \\
I_{d s a t} \frac{V_{d s}}{V_{d s a t}} & V_{d s}<V_{d s a t} \\
I_{d s a t} & V_{d s}>V_{d s a t}
\end{array}\right\} V_{g s}>V_{\text {th }} \quad \begin{aligned}
& \text { Cutoff } \\
& \text { Linear } \\
& \text { Saturation }
\end{aligned}
$$

where

$$
\begin{gathered}
I_{d s a t}=P_{c} \frac{\beta}{2} V_{G T}^{\alpha} \\
V_{d s a t}=P_{v} V_{G T}^{\alpha / 2} \\
\beta=\mu C_{o x} \frac{W}{L} ; \quad V_{G T}=V_{g s}-V_{t h} ; \quad C_{o x}=\frac{\epsilon_{o x}}{t_{o x}}
\end{gathered}
$$

$P_{c}, P_{v}, \alpha$ are three parameters that can be determined from curve fitting of I-V plot. Parameter $\alpha$ is called the velocity saturation index, $\mu$ is the mobility of mobile charges, $C_{o x}$ is the gate-oxide capacitance per unit area, $\epsilon_{o x}$ is the permittivity of $\mathrm{SiO}_{2}, t_{o x}$ is oxide thickness, $V_{g s}$ is the gate-to-source voltage, and $V_{t h}$ is the threshold voltage of the transistor.

In transistors with long channels or low $V_{D D}, \alpha$ reaches 2 and they display a quadratic $\mathrm{I}-\mathrm{V}$ characteristic in the saturation region as proposed by Shockley. As transistors become shorter, velocity saturation prevails and $\alpha$ goes toward 1 .

In both, the proposed model by Shockley and Sakurai, the current for $V_{g s}<V_{t h}$ (i.e., Sub-threshold region) is considered to be 0 . However, in real transistors, even in this region there is a current flowing from the drain to source. For circuits operating in the super-threshold mode (i.e., $V_{g s}>V_{t h}$ ), the sub-threshold currnet is considered a leakage. 
But for low-power or ultar low-power applications, operating in the sub-threshold region, this small current is the main operatio current. In the remaining part of this section a brief introduction of the sub-threshold current is presented.

The sub-threshold current may be expressed as [15]

$$
I_{d s}=\beta v_{T}^{2} e^{1.8} e^{\frac{V_{g s}-V_{t h}}{n v_{T}}}\left(1-e^{-\frac{V_{d s}}{v_{T}}}\right)
$$

where $\beta$ is introduced in equation (3-2), $v_{T}=K T / q$ is the thermal voltage, and $n$ is the sub-threshold slope factor that varies by the depletion region characteristics and is typically in the range of 1.3 to 1.7. Parameter $n$ is expressed as [77]

$$
n=1+\frac{C_{d e p}}{C_{o x}}
$$

where $C_{d e p}$ denotes the capacitance per unit area of the depletion layer under the gate area. Parameter $n$ can be rewritten as

$$
n=1+\frac{C_{d e p}}{C_{o x}}=1+\frac{\epsilon_{S i} / W_{d e p}}{\epsilon_{o x} / t_{o x}} \approx 1+3 \frac{t_{o x}}{W_{d e p}}
$$

where $W_{d e p}$ is the width of depletion region under the gate and $\epsilon_{S i}$ is the permittivity of Silicon.

Equation (3-3) reveals two interesting properties. First, as $V_{d s}$ exceeds a few $v_{T}$, $1-e^{-\frac{V_{d s}}{v_{T}}}$ becomes 1 and the current becomes independent of $V_{d s}$. Second, the slope of $I_{d s}$ on a semi-logarithmic scale equals

$$
\frac{\partial\left(\log _{10} I_{d s}\right)}{\partial V_{g s}}=\left(\log _{10} e\right) \frac{1}{n v_{T}}
$$

The inverse of this quantity is called the sub-threshold slope, $S$

$$
S=n v_{T} \operatorname{Ln} 10=2.3 v_{T}\left(1+3 \frac{t_{o x}}{W_{d e p}}\right) \quad \mathrm{V} / \mathrm{dec}
$$


In order to turn off the transistor by lowering $V_{g s}$ in the sub-threshold region, $S$ must be as small as possible. Parameter $S$ is typically in the range of 70 to $100 \mathrm{mV} / \mathrm{dec}$. In the extreme case where the oxide thickness reaches zero, sub-threshold slope reaches $60 \mathrm{mV} / \mathrm{dec}$ at room temperature [46]. In Figure 3.2 the current versus $V_{\mathrm{gs}}$ is plotted in semi- logarithmic scale. The three regions of operation and the sub-threshold slope are identified.

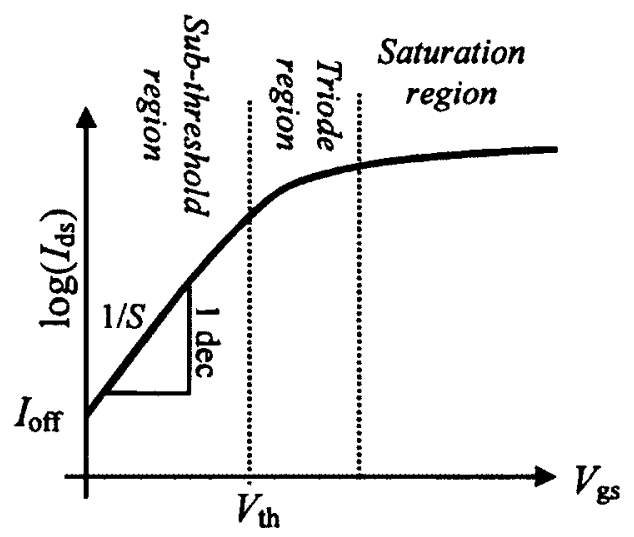

Figure 3.2 Current vs. $V_{g}$ in logarithmic scale.

The magnitude of $S$ limits the threshold voltage scaling. For example for $S=100 \mathrm{mV} / \mathrm{dec}$ and the threshold voltage of $200 \mathrm{mV}$, the "on current" is only two orders of magnitude higher than the "off current", which results in low noise margins and a weak performance for digital circuits.

Comparing Equations (3-2) and (3-3) shows that in the super-threshold region, the current has a nearly linear relation with the threshold voltage, while its relation to the threshold voltage in the sub-threshold region is an exponential relation. This implies that any small changes in the threshold voltage do not have a significant effect on the current in the super-threshold region. However, in the sub-threshold region, a small change in the threshold voltage causes a big change in the current. More detailed studies on the current behaviour are presented in Chapter 4 . 


\subsubsection{Threshold Voltage}

To have a better understanding of the effects of transistor sizing on the threshold voltage and, consequently, on the current, a simple quantitative expression is introduced for the threshold voltage [78]

$$
V_{t h}=V_{f b}+\emptyset_{s t}+\frac{Q_{d e p}}{C_{o x}}
$$

where $V_{f b}$ is the flat-band voltage, $\emptyset_{s t}$ is surface potential at the threshold edge, $Q_{d e p}$ is the depletion region charge. The first and second terms in Equation (3-8) are fixed for a given technology and depend on the doping levels of the substrate and poly silicon [79]. But the third term dependent on the transistor sizes. It means that changing the size of a transistor changes its threshold voltage. Four important phenomena that relate the threshold voltage variations to the transistor dimensions are introduced in next sections.

\subsubsection{Effect of Channel Width}

A decrease in the channel width changes the threshold voltage and, as a result, changes the sub-threshold current. There are mainly two ways by which the channel width modulates the threshold voltage: Narrow-Width Effect (NWE) and Inverse NarrowWidth Effect (INWE).

NWE: In older technologies where Local Oxidation of Silicon (LOCOS) is used to isolate two adjacent transistors, the existence of fringing field extends the depletion region to outside of the defined channel width. Hence, the depletion charge in the bulk increases. According to Equation (3-8), this causes a rise in threshold voltage, as shown in Figure 3.3(a). This effect becomes more prominent as the channel width decreases, and the depletion region under the fringing field becomes comparable to the depletion region formed under the gate by the vertical field.

The second method by which the NWE changes the threshold voltage is the higher doping level at the edge of the channel due to the encroachment of the channel stop dopants under the gate. Thus, a higher voltage is needed to completely invert the channel [80]. 
INWE: As integration density increased in CMOS digital circuits, LOCOS technology caused problem because of the so called "bird's beak" phenomenon, due to the lateral oxidation. Shallow Trench Isolation (STI) with a vertical field oxide, improves the area efficiency in device isolation. As depicted in Figure 3.3(b), extensive gathering of the fringing field lines appears on the side of the depletion region under the gate. This phenomenon can be modeled as an effective increase in gate oxide capacitance [46]. According to Equation (3-8), this increase in gate oxide with constant $Q_{\text {dep }}\left(\Delta Q_{d e p} \approx 0\right)$ causes a reduction in the threshold voltage as the transistor width becomes narrower.

Therefore, we note the LOCOS cause a threshold roll-up while STI causes a threshold roll-off as he channel width decreases.
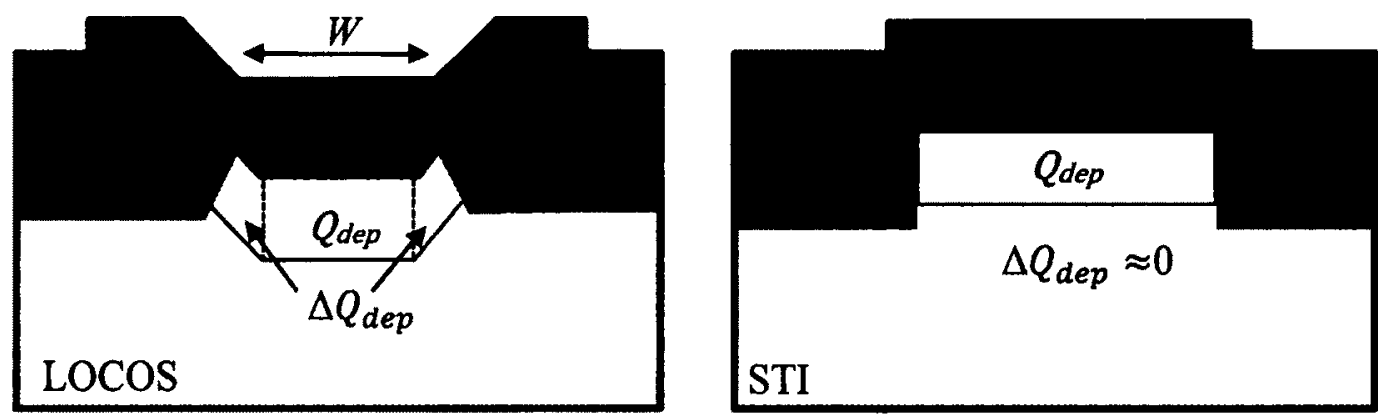

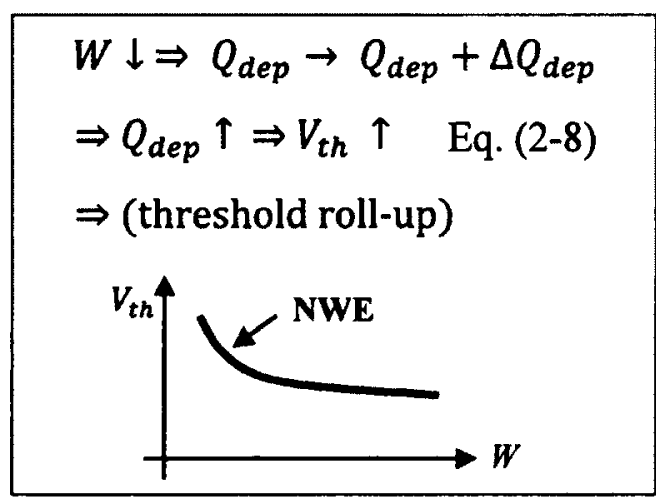

(a)

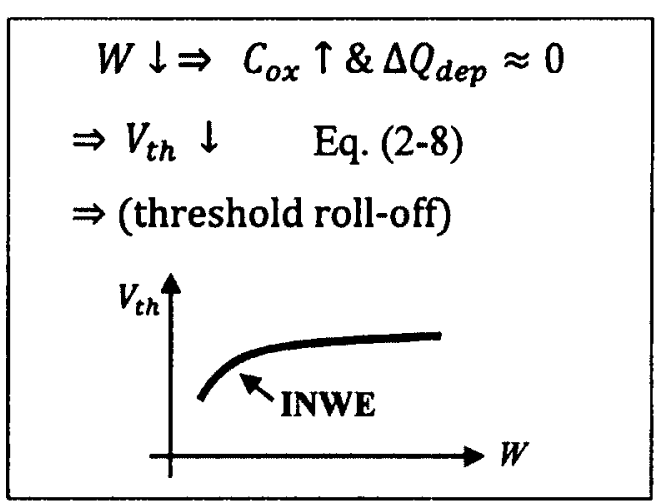

(b)

Figure 3.3 Cross-section of a MOS transistor along the width showing LOCOS (a) and STI (b) isolation and their effect on threshold voltage. 


\subsubsection{Effect of Channel length}

The channel length has its own effect on the threshold voltage. Two main phenomena that the channel length impacts the threshold voltage come from the SCE and the RCSE.

SCE: In devices with long channels, the gate is completely responsible for depleting the substrate to produce $Q_{d e p}$. In very short channel devices, part of the depletion is accomplished by merging the depletion regions of the source and the drain with the depletion region under the gate, as shown in Figure 3.4. Hence, lower $V_{\mathrm{gs}}$ is required to deplete the substrate, i.e., decreasing the channel length decreases the threshold voltage. This phenomenon is referred as charge sharing between the source and drain depletion regions and the channel depletion region.

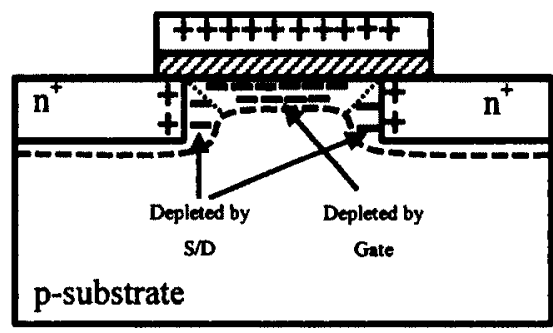

$$
\begin{aligned}
& L \downarrow \Rightarrow \text { Charge sharing between } S \& D \text { depletion region and Channel depletion } \\
& \Rightarrow Q_{\text {dep }} \downarrow \Rightarrow V_{\text {th }} \downarrow \\
& \Rightarrow \text { (threshold roll-off) }
\end{aligned}
$$

Figure 3.4 Charge sharing between source/drain depletion regions and the channel depletion region resulting in threshold roll-off.

Another SCE phenomenon related to the threshold voltage is drain-induced barrier lowering (DIBL). As the drain voltage increases, the channel becomes more attractive for the mobile charges. In other words, the potential barrier for the mobile charges is lowered as shown in Figure 3.5. This results in lowering the threshold voltage lowering. As the channel length become shorter, DIBL becomes more noticeable.

DIBL has a couple of undesirable effects that degrade the circuit performance. First, DIBL reduces the output impedance, which is not desirable in most analog 
applications [77]. Second, at extremely short channel lengths, DIBL causes the gate voltage to fail in turning off the transistor completely. This means more leakage current from the drain to source even when the transistor is in the "off" state [81].

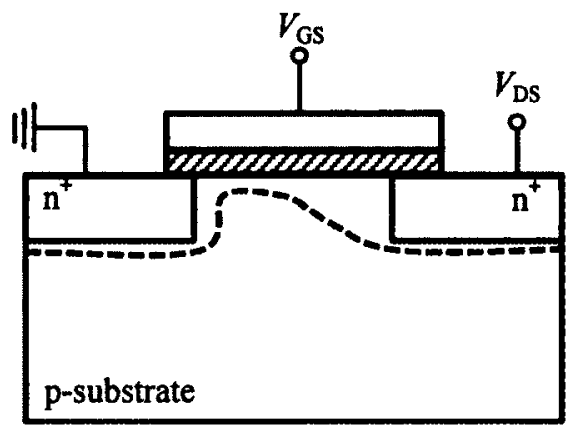

$$
\begin{aligned}
& V_{D S} \uparrow \Rightarrow \text { Depletion region widen near drain } \\
& \Rightarrow\left\{\begin{array}{c}
Q_{\text {dep }} \text { in drian vicinity } \uparrow \\
Q_{\text {dep }} \propto \text { surface potential }
\end{array} \Rightarrow \text { surface potential } \uparrow\right. \\
& \Rightarrow \text { Voltage Barrier for electrons from source to drian } \downarrow \Rightarrow V_{t h} \downarrow \\
& \Rightarrow \text { (threshold roll-off) }
\end{aligned}
$$

Figure 3.5 DIBL in a short-channel device.

RCSE: Both "charge sharing of the source/drain depletion region and the channel depletion region" and "DIBL" are particularly pronounced in lightly doped substrates [46]. To mitigate these undesired phenomena, which make a threshold roll-off as the channel length decreases, in modern CMOS technologies non-uniform $\mathrm{p}^{+}$HALO doping in the source-body and drain-body boundaries are used. More highly doped substrate near the edge of the channel reduces the charge sharing effects from source and drain depletion regions. Also, these highly doped regions at the channel edges make the junction depletion widths smaller [8]. This reduction in the depletion region width close to the source and drain junctions make the distance between the source and drain longer, which leads to a reduction in the DIBL phenomenon. Although HALO implementation supresses the charge-sharing and DIBL effects on the threshold roll-off as the channel length decreases, it has its side effects. One of the side effects which is related to the threshold voltage, is the threshold roll-up as 
the channel length decreases. When the channel length becomes shorter, HALO regions in the source and drain vicinities merge together and causes an increase in the doping level under the gate in the channel area. It means that for depleting the surface, a larger gate voltage is needed. As the channel length becomes longer and the distance between the HALO regions increases, the surface doping decreases along the channel, which causes the threshold voltage reduction. The effect of HALO doping in a short-channel device and in a long one is illustrated in Figure 3.6.

Note that the HALO is not only near the source/drain, but also it is underneath the inversion channel. By doing this, the effect on threshold is minimal.

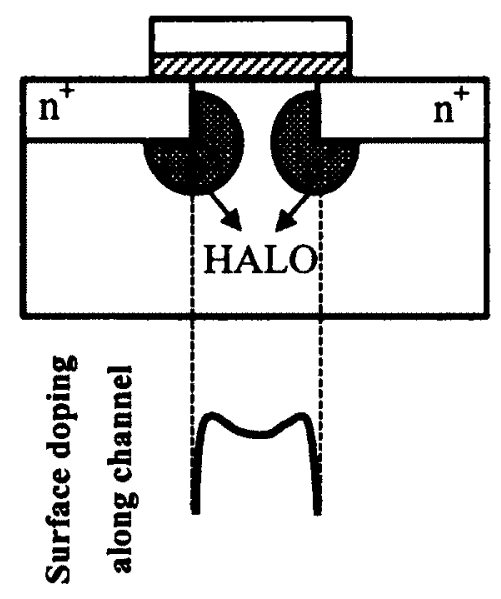

Short-channel

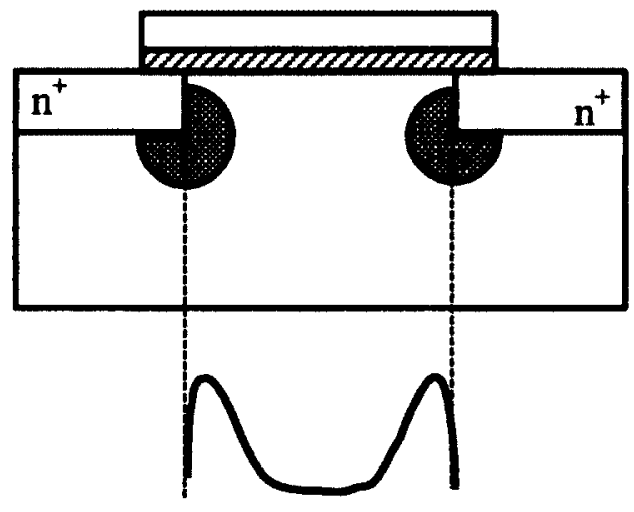

Long-channel

NMOS

NMOS
$L \downarrow \Rightarrow$ HALOs merge $\Rightarrow$ High surface doping $\Rightarrow Q_{\text {dep }} \uparrow \Rightarrow V_{\text {th }} \uparrow$
$L \uparrow \Rightarrow$ HALOs seperate $\Rightarrow$ Low surface doping $\Rightarrow Q_{\text {dep }} \downarrow \Rightarrow V_{\text {th }} \downarrow$

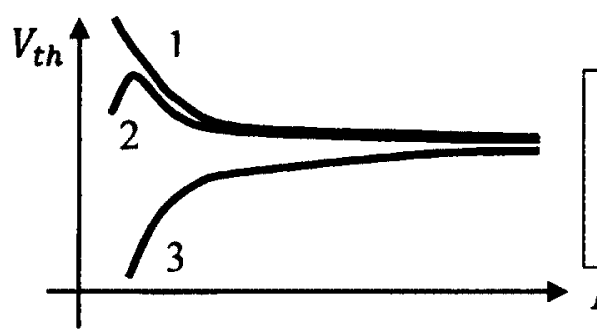

1 with HALO, negligible DIBL (small $V_{D S}$ )

2 with HALO, noticeable DIBL (large $V_{D S}$ )

3 without HALO

Figure 3.6 HALO doping effects on threshold voltage of short and long-channel transistors. 


\subsubsection{Capacitances}

In order to understand the dynamic behaviour of MOSFETs, beside the current and the threshold voltage, we need to study MOSFET different capacitances.

Between every two of the four terminals of an MOSFET a capacitance exists, as illustrated in Figure 3.7. The capacitance between source and drain, however, is negligible [15]. Figure 3.8 relates the capacitances to the geometry of an MOSFET.

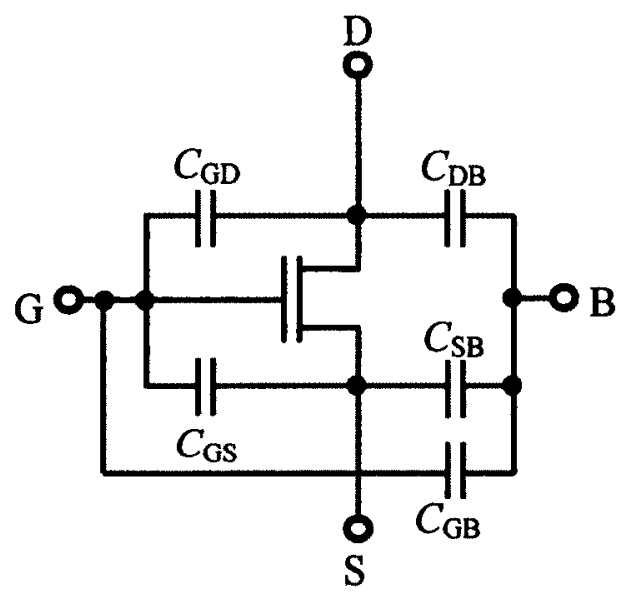

Figure 3.7 MOSFET Capacitances.

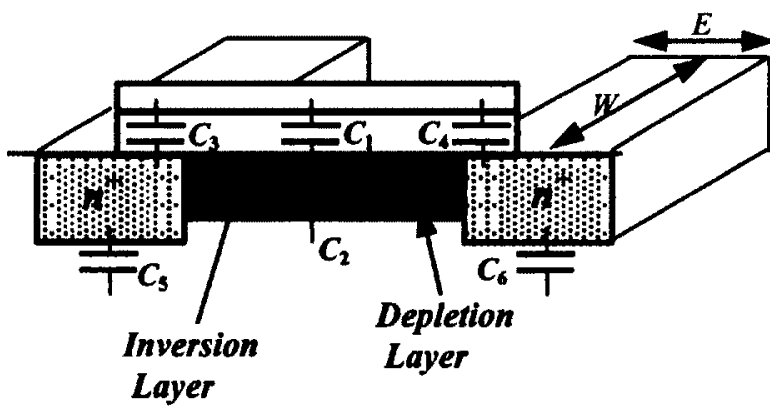

(a)

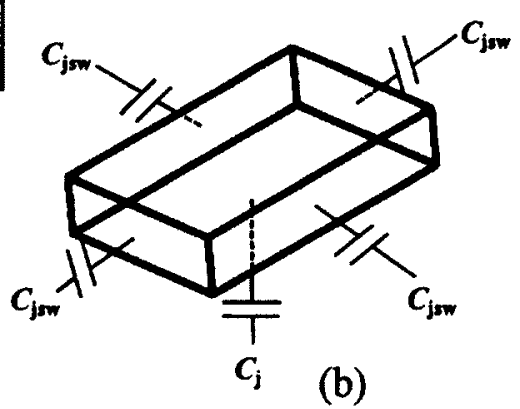

(b)

Figure 3.8 (a) Representation of MOSFET capacitances, (b) decomposition of source/drain junction capacitance to bottom and sidewall components. 
According to Figure 3.8, the capacitances shown can be explained as below

- $C_{1}$ : Oxide capacitance between the gate and the channel, expressed as

$$
C_{1}=C_{o x} W L=W L \frac{\epsilon_{o x}}{t_{o x}}
$$

- $C_{2}$ : Depletion capacitance between the channel and the substrate, defined as

$$
C_{2}=C_{d e p} W L=W L \frac{\epsilon_{S i}}{W_{d e p}}
$$

where $W_{d e p}$ is the width of depletion layer given by $W_{\text {dep }}=\sqrt{\left(2 \epsilon_{s i} \emptyset_{s}\right) /\left(q N_{s u b}\right)}[15], \emptyset_{s}$ is the surface potential and $N_{\text {sub }}$ is the substrate doping.

- $C_{3}$ and $C_{4}$ : Gate capacitances to source/drain due to the overlap of the gate ploy with source/drain. Because of fringing field, these capacitances cannot be simply written as $W L_{D} C_{o x}$. In MOSFET models, $C_{g d o v}$ and $C_{g s o v}$ represent the overlap capacitances per unit width.

- $C_{5}$ and $C_{6}$ : Junction capacitances between source/drain and the substrate. As illustrated in Figure 3.8 (b), these capacitances are divided into the bottom and sidewall capacitances. They depend on both the area and perimeter of the source/drain. The area is $A=W E$ and $P=2(W+E)$ (Figure 3.8). The total junction capacitance is

$$
C_{5}=C_{6}=C_{j} A+C_{j s w}(P-W)+C_{j s w g} W
$$

where $C_{j}$ is the capacitance per unit area between the bottom of junction and the substrate, $C_{j s w}$ is the capacitance per unit length between the 3 sidewalls of the junction and the substrate that are not facing the channel, and $C_{j s w g}$ is the capacitance per unit length between the sidewall of the junction and the substrate that faces the channel. All these capacitances $\left(C_{j}, C_{j s w}\right.$, and $\left.C_{j s w g}\right)$ are functions of the source/drain voltages. For example, $C_{j}$ may be expressed as [77]

$$
C_{j}=C_{j 0}\left(1+\frac{V_{R}}{\Psi_{b}}\right)^{-M_{j}}
$$


where $C_{j 0}$ is the junction capacitance at zero bias, $\Psi_{b}$ is the built-in voltage, $M_{j}$ is the junction grading coefficient, and $V_{\mathrm{R}}$ is the reverse voltage across the junction. $C_{j s w}$ and $C_{j s w g}$ can be expressed similar to (3-12), but $C_{j 0}$ must be replaced by $C_{j s w 0}$ and $M_{j}$ with $M_{j s w}$ or $M_{j s w g}$.

Table 3.1 summarize the relation between the capacitances in Figure 3.7 and Figure 3.8.

Table 3.1 Approximation for MOSFET capacitances [80].

\begin{tabular}{|c|c|c|c|c|}
\hline $\begin{array}{c}\text { Figure } 3.7 \\
\text { Capacitances }\end{array}$ & $\begin{array}{c}\text { Figure } 3.8 \\
\text { Capacitances }\end{array}$ & \multicolumn{3}{|c|}{ Relating Expression } \\
\hline$C_{D B}, C_{S B}$ & $C_{5}, C_{6}$ & \multicolumn{3}{|c|}{$C_{\mathrm{j}} A+C_{\mathrm{jsw}}(W+2 E)+C_{\mathrm{jswg}} W$} \\
\hline & & \multicolumn{3}{|c|}{ Region of operation } \\
\hline & & OFF & Triode & Saturation \\
\hline$C_{G S}$ & $C_{3}, C_{4}$ & $C_{g s o v} W$ & $C_{1} / 2+C_{g s o v} W$ & $2 C_{1} / 3+C_{g s o v} W$ \\
\hline$C_{G D}$ & $C_{3}, C_{4}$ & $C_{g d o v} W$ & $C_{1} / 2+C_{g d o v} W$ & $C_{g d o v} W$ \\
\hline$C_{G B}$ & $C_{1}, C_{2}$ & $C_{1} C_{2} /\left(C_{1}+C_{2}\right)$ & 0 & $\begin{array}{llll}0 & & & \\
\end{array}$ \\
\hline
\end{tabular}

All capacitances connected to the gate of the MOSFET should be modeled for delay calculations. Defining $C_{G G}$ as the total gate capacitance, we may write

$$
C_{G G}=C_{G S}+C_{G D}+C_{G B}
$$

Due to the dependence of $C_{G S}, C_{G D}$, and $C_{G B}$ to the gate voltage, $C_{G G}$ is also a function of the gate voltage as depicted in Figure 3.9 [77].

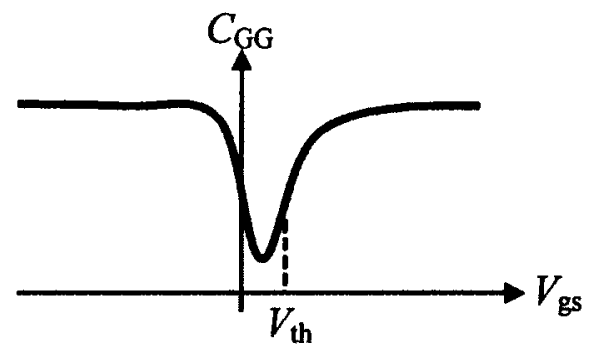

Figure 3.9 Dependence of gate capacitance of an NMOS transistor to gate voltage. 


\subsubsection{Leakage Currents}

A static CMOS circuit consumes power even when it is in its idle mode and no switching activities take place. The reason for this power consumption is the leakage currents, which are not negligible in modern CMOS technologies due to the extensive down scaling of transistors' dimensions. There are four major sources of leakage currents in CMOS transistors.

\section{Sub-threshold Leakage ( $\left.I_{\text {ofr }}\right)$}

The sub-threshold leakage current is the drain to source current of a transistor when the transistor operates in the weak-inversion mode. The magnitude of this current can be calculated from Equation (3-3) for $V_{\mathrm{gs}}=0$ and $V_{\mathrm{ds}}=V_{\mathrm{DD}}$ (transistor being off), which is presented in Figure 3.2. The magnitude of this leakage current is a function of the temperature, supply voltage, device size, and the process parameters [46].

\section{Gate Oxide Tunneling Leakage $\left(I_{\mathbf{G}}\right)$}

The downscaling of the oxide thickness increases the electric field across the gate oxide resulting in electron tunneling from gate to the substrate or the source and drain. Two mechanisms are responsible for this phenomenon: Fowler-Nordheim tunneling and direct tunneling, where the latter is dominant at lower voltages and thinner oxide [15]. Gate oxide leakage for NMOS transistor is higher than PMOS transistor. Tunneling current decreases exponentially with the oxide thickness and becomes more significant for technologies beyond $100 \mathrm{~nm}$ [15].

\section{Reverse-biased Junctions Leakages ( $I_{\text {Bulk }}$ )}

Though the $p-n$ junctions between the source/drain to the substrate and the well to the substrate are usually reverse biased, yet small amount of currents leak via these junctions. The magnitude of this leakage current depends on the area of source/drain and well diffusions and doping concentration. The highly doped shallow junctions and HALO doping necessary to control SCE (Section 3.1.2.2) in the nanometer devices has worsened this leakage current [46]. 


\section{Gate Induced Drain Leakage (I $\mathbf{I}_{\text {GIDL }}$ )}

This leakage current is caused by high electric field in the drain junction of MOSFETs. HALO doping in the vicinity of the drain junction, which is done to control punch-through and DIBL in nano-scale technologies, results in band-to-band tunnelling current at the drain edge, especially as the drain-bulk voltage increases. Thinner oxide and higher supply voltage increases GIDL current. GIDL occurs where the gate overlaps the drain, hence, it is a function of transistors width [15]. When a circuit operates in the sub-threshold region, voltages on the gate and the drain are not so relatively high. This means that in the sub-threshold region of operation the GIDL current may be neglected.

The most significant leakage current among the leakage currents is the sub-threshold leakage current. In Chapter 4 a comparative study of the leakage currents is presented for different technology nodes.

\subsection{Quality Metrics of a Digital Circuit}

This section defines a set of basic features of a digital circuit to quantify the quality of a design. The relative importance of these metrics depends on the application of the circuit. For example for a desktop computer speed is a crucial property, while for a mobile electronic device the energy consumption is the dominant metric. These introduced properties give a good understanding for designing a digital circuit based on special constraints.

\subsubsection{Propagation Delay}

The propagation delay for a logic gate is the time taken for a signal to propagate from the input to the output node. This delay is defined as the time from when the input signal passes $V_{\mathrm{DD}} / 2$ until the corresponding output signal passes $V_{\mathrm{DD}} / 2$, as depicted in Figure 3.10. The capacitance shown in Figure 3.10 includes the drain junction capacitance of the first inverter and the gate capacitance for the second inverter which is acting as a load. 
All these capacitances are non-linear functions of the output voltage as described in Section 3.1.3.
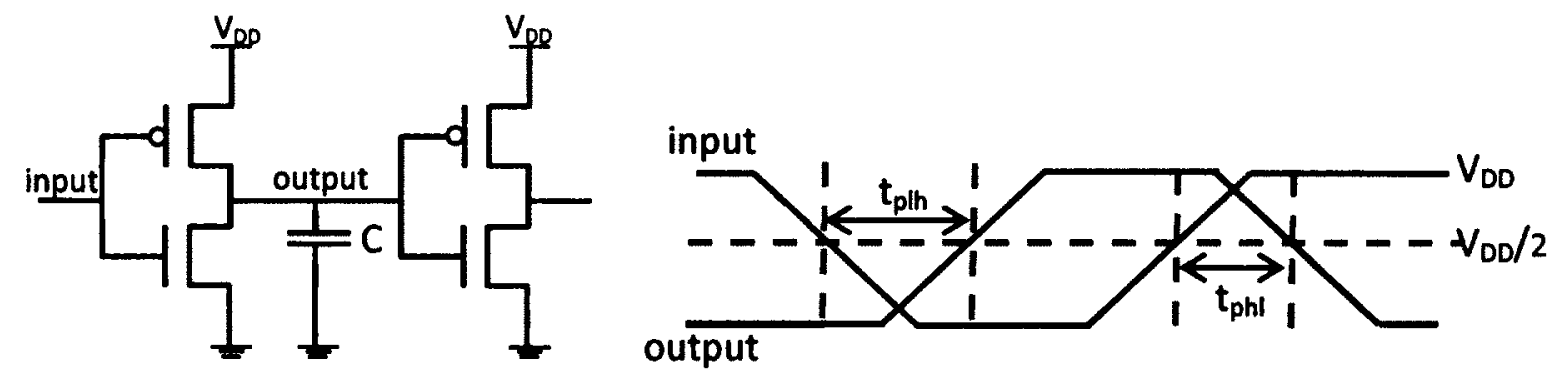

Figure 3.10 Propagation delay for an inverter driving another inverter with input and output signals approximated as ramps.

To calculate these delays, one should solve a differential equation based on charging or discharging of the capacitances in the circuit. If current $i(t)$ is charging or discharging capacitance $C$, it is related to the voltage across the capacitance by

$$
i(t)=C \frac{d V}{d t}
$$

Since both the current and capacitances are functions of $V$, solving the equation (3-14) is not easy. But, it can be simplified by making some assumptions. If $d V$ replaced by $\Delta V=V_{D D} / 2$ and $d t$ by $t_{\mathrm{plh}}$ or $t_{\mathrm{phl}}$, equation (3-14) will be simplified to

$$
I_{a v}=C_{a v} \frac{V_{D D} / 2}{t_{p l h}\left(\text { or } t_{p h l}\right)}
$$

where $I_{a v}$ and $C_{a v}$ are the average values of the current and capacitance between the start of the transition and the end of transition. Although the current and the capacitances are non-linear functions of voltage and averaging seems not an accurate method, the simplified equation is still a valid approximation for delay estimation [15], especially in the sub-threshold region where the supply voltage is in a few hundred millivolts range and voltage variation is not large.

Solving (3-15) for $t_{p h h}$ or $t_{p h l}$ results in two equations for $t_{p l h}$ and $t_{p h l}$ as

$$
t_{p l h}=\frac{C_{a v} V_{D D}}{2 I_{P a v}}, \quad t_{p h l}=\frac{C_{a v} V_{D D}}{2 I_{N a v}}
$$


where $I_{P a v}$ and $I_{N a v}$ are the PMOS and NMOS transistors average currents, respectively. The propagation delay $t_{p}$ is defined as the average of the two factor:

$$
t_{p}=\frac{t_{p l h}+t_{p h l}}{2}=\frac{C_{a v} V_{D D}}{4}\left(\frac{1}{I_{N a v}}+\frac{1}{I_{P a v}}\right)
$$

To optimize the propagation delay, according to Equation (3-17), the effect of transistor sizing should be considered on $C / I$ ratio. A detailed study on the delay optimization is presented in Chapter 5.

\subsubsection{Power Consumption}

Power dissipation in a CMOS circuit has two components: dynamic and static dissipation.

Dynamic power arises from

- Switching power consumption that refers to the power consumption due to the charging and discharging of a capacitance, $C$, and expressed as

$$
P_{s w}=\alpha C V_{D D}^{2} f
$$

where $f$ is operation switching frequency and $\alpha$ is the switching activity factor. The switching activity factor is the probability that a node makes a transition from 0 to 1 . Only in this transition a node consumes the power extracted from the voltage supply.

- "Short-circuit" current power consumption while both pull-down and pull-up transistors are partially $\mathrm{ON}$, and may be written as

$$
P_{s c}=l_{s c-a v} V_{D D}
$$

where $I_{s c-a v}$ is the average short-circuit current. It is important to keep the signal edges fall and rise fast to have negligible $P_{s c}$.

Putting these two together gives the total dynamic power consumption of a circuit 


$$
P_{D Y N}=\underbrace{\alpha C V_{D D}^{2} f}_{P_{s w}}+\underbrace{I_{S c-a v} V_{D D}}_{P_{s c}}
$$

The dynamic power consumption mostly consists mostly of the switching power and the short-circuit power dissipation is normally less than $10 \%$ of the whole [15]. Hence, as $V_{D D}$ has a quadratic relation to $P_{D Y N}$, it is important to select the minimum $V_{D D}$ that meets the required frequency of operation.

The static power consumption is caused by leakage currents and may be expressed as

$$
P_{S T}=\left(I_{\text {off }}+I_{G}+I_{B u l k}\right) V_{D D}
$$

where all leakage currents are described in Section 3.1.4.

\subsubsection{Energy Consumption}

In an inverter when the output makes a transition from $0 \rightarrow V_{D D}$, the dynamic energy drawn from the power supply in one cycle is

$$
E_{D Y N}=C V_{D D}^{2}
$$

For this transition, the stored energy in the load capacitance is

$$
E=\frac{1}{2} C V_{D D}^{2}
$$

This means that during this transition, $50 \%$ of the total energy drawn from the supply is consumed by the PMOS transistor. For the output transition $V_{D D} \rightarrow 0$, the stored energy in the capacitor is consumed by the NMOS transistor and no energy is drawn from the power supply.

The other component of the consumed energy is the static or leakage energy in one cycle, which may be expressed as

$$
E_{S T}=I_{L e a k} V_{D D} t_{p}
$$

where $t_{p}$ is the propagation delay or the needed time to complete one computation and $I_{\text {Leak }}$ is the total leakage current. 
Substituting $t_{p}$ from Equation (3-17) to Equation (3-24), the leakage consumed energy becomes

$$
E_{S T}=I_{\text {Leak }} V_{D D} \frac{C V_{D D}}{2 I_{\text {on-av }}}
$$

Putting Equations (3-22) and (3-25) together, the total energy consumption comes to

$$
E_{T}=C V_{D D}^{2}\left(1+\frac{I_{\text {Leak }}}{2 I_{\text {on-av }}}\right)
$$

Equation (3-26) shows a quadratic relation between the total consumed energy and the supply voltage. Hence, decreasing $V_{D D}$ decreases the total energy quadratically. However, decreasing $V_{D D}$ decreases $I_{o n-a v}$ too. In the other words, decreasing $V_{D D}$ increases $E_{S T}$ while decreases $E_{D Y N}$. So, it is predictable that the consumed energy shows a minimum point with respect to $V_{D D}$ as shown in Figure 3.11. In [1] and [36], the authors show that the minimum energy point occurs at a $V_{D D}$ in the sub-threshold or nearthreshold region.

The minimum energy point typically consumes an order of magnitude less energy than the conventional operation point; however it operate at least 1000 times slower [15].

Accordingly, there is a trade-off between the optimum propagation delay and the

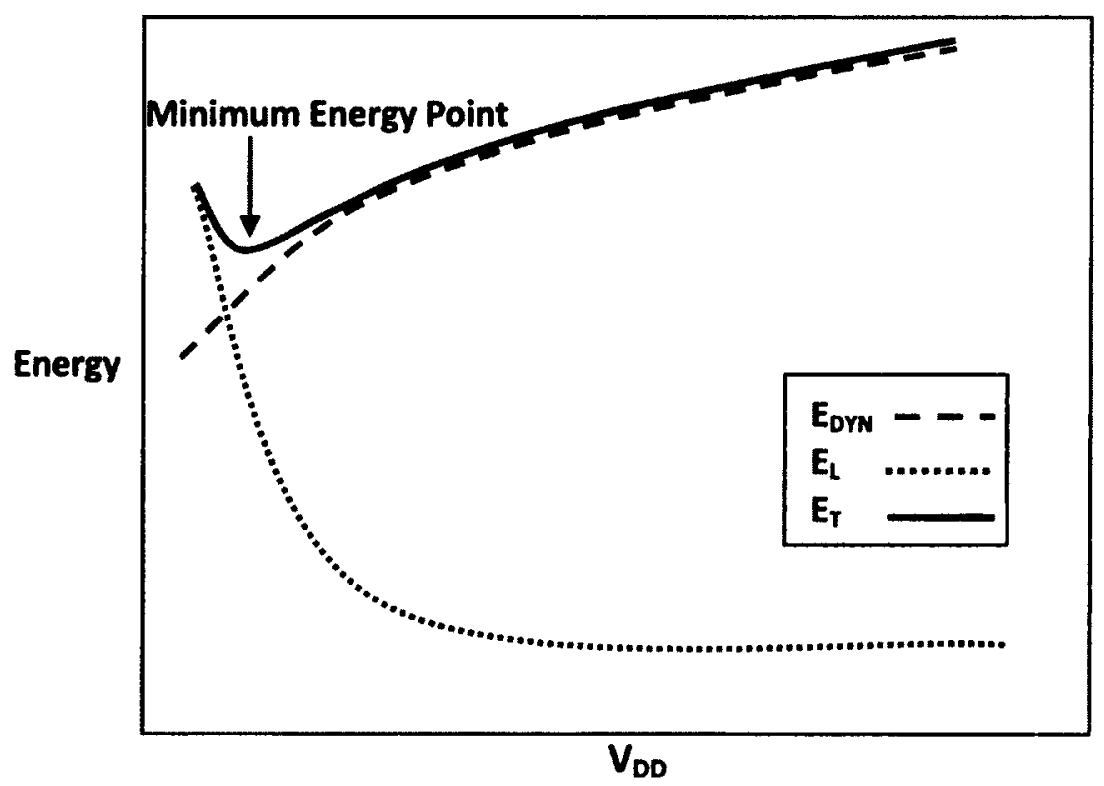

Figure 3.11 Minimum energy point 
optimum power and energy consumptions. Hence, two metrics are used in digital circuits design to combine the delay with each of power or energy consumption. One of these metrics is the power-delay product (PDP). The PDP is simply the consumed energy during one switching activity; so PDP is expressed in the unit of energy (J).

The other important metric in digital circuits design is the energy-delay product (EDP), and is considered the conclusive metric to define the quality of a digital circuit [82]. The EDP has a unit of joules-second (J.s).

\subsubsection{Voltage Transfer Characteristics}

The quality of a logic gate is often measured using the Voltage-Transfer Characteristics (VTC) that is a plot of the output voltage as a function of the input(s). From such a graph, the figures of merit of the logic gate, such as the noise tolerance, can be extracted. As an example, VTC of an inverter is shown in Figure 3.12. A few key values are indicated on the graph to help us define some figure of merits.

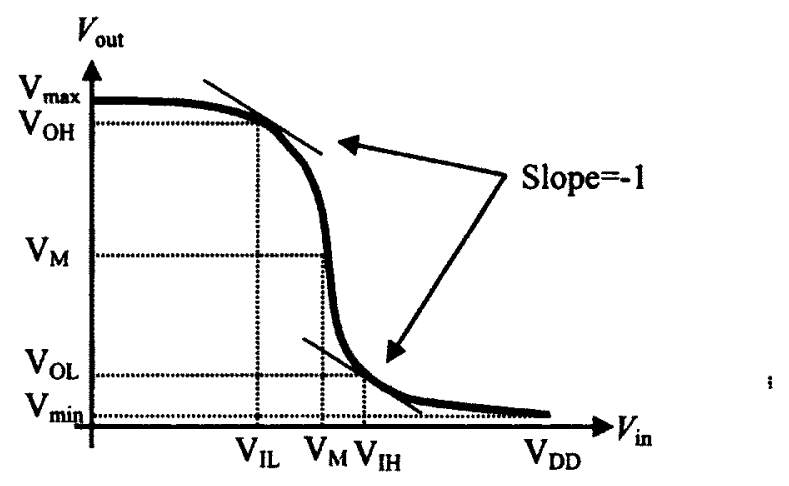

Figure 3.12 Typical inverter VTC.

The definitions for the voltages shown in Figure 3.12 follow

$V_{\mathrm{OH}}$ : The minimum output voltage of an inverter that indicates a logic " 1 "

$V_{\mathrm{OL}}$ : The maximum output voltage of an inverter that indicates a logic " 0 "

$V_{\mathrm{IL}}$ : The maximum input voltage to an inverter that is considered a logic " 0 "

$V_{\mathrm{IH}}$ : The minimum input voltage to an inverter that is considered a logic "1" 
From the VTC, one can see that if $V_{\mathrm{in}}<\mathrm{V}_{\mathrm{IL}}$, the output voltage is a valid logic 1, and, similarly, if $V_{\mathrm{in}}>\mathrm{V}_{\mathrm{IH}}$, the output voltage is a valid logic 0 .

When cascading inverters, we can define the noise margin high, $N M \mathrm{H}$ that ensures a logic " 1 " output from the first inverter is interpreted as a logic " 1 " input for the second inverter. Similarly, we can define the noise margins low, NML that ensures a logic "0" output from the first inverter is interpreted as a logic " 0 " input for the second inverter. The expressions for these noise margins are given by

$$
\begin{gathered}
N M H=V_{O H}-V_{I H} \\
N M L=V_{I L}-V_{O L}
\end{gathered}
$$

Static Noise Margin (SNM) is defined as the minimum of the two noise margins expressed in Equation (3-27).

\subsection{Chapter Summery}

In this chapter we reviewed most of the material that we need in the following chapters to study the effect of transistor sizing either on the transistor's behaviour by itself or digital circuits' optimization.

In Chapter 4, the effect of transistor sizing on MOSFET characteristics in the subthreshold region, such as the threshold voltage, current, and capacitances are studied in detail for different available CMOS technology kits. Based on what discussed in Chapter 4 , in Chapter 5 a method for delay optimization is presented. 


\section{MOSFET Behavior in Sub-threshold Region}

To have a better understanding of transistor-sizing effects on a digital circuit performance and its power consumption in the sub-threshold region, the behavior of a transistor must be studied individually in this region of operation. The main important characteristics of a transistor that should be studied are: threshold voltage, transistor ON and OFF (leakage) currents, capacitances, and sub-threshold slope. In this chapter the effect of the channel length and width on these parameters are studied. The studied CMOS technology nodes are: TSMC $180 \mathrm{~nm}$, IBM $130 \mathrm{~nm}$, TSMC $90 \mathrm{~nm}$, and TSMC $65 \mathrm{~nm}$ LP. In the TSMC $65 \mathrm{~nm}$ technology node, two flavors of transistors are provided: low-threshold (lvt) and standard-threshold (svt). In each technology node an investigation has been done on the NMOS and PMOS transistors.

\subsection{Threshold Voltage Variation}

Due to the exponential relation between the sub-threshold current and the threshold voltage, as shown in Equation (3-3), a small change in the threshold voltage causes a significant change in the sub-threshold current. Hence, any changes on threshold voltage caused by the transistor dimensions must be considered to predict the behavior of the current in this mode of operation.

As explained in Section 3.1.2, in older technologies, where LOCOS is used for transistors isolation and HALO doping is not used, a roll-up and roll-off of the threshold voltage has been seen with the channel width and channel length reduction, respectively. On the contrary, in the modern technologies, which LOCOS is replaced with STI and HALO doping is used to mitigate the DIBL and punch through in very short channel devices, a roll-off and roll-up of the threshold voltage is seen for a decrease in the channel width and length, correspondingly. Figure 4.1 shows the threshold voltage dependence on the channel length and channel width for the NMOS and PMOS 
transistors in IBM $130 \mathrm{~nm}$ CMOS technology. The left plot shows the INWE, while the right plot represents RSCE.
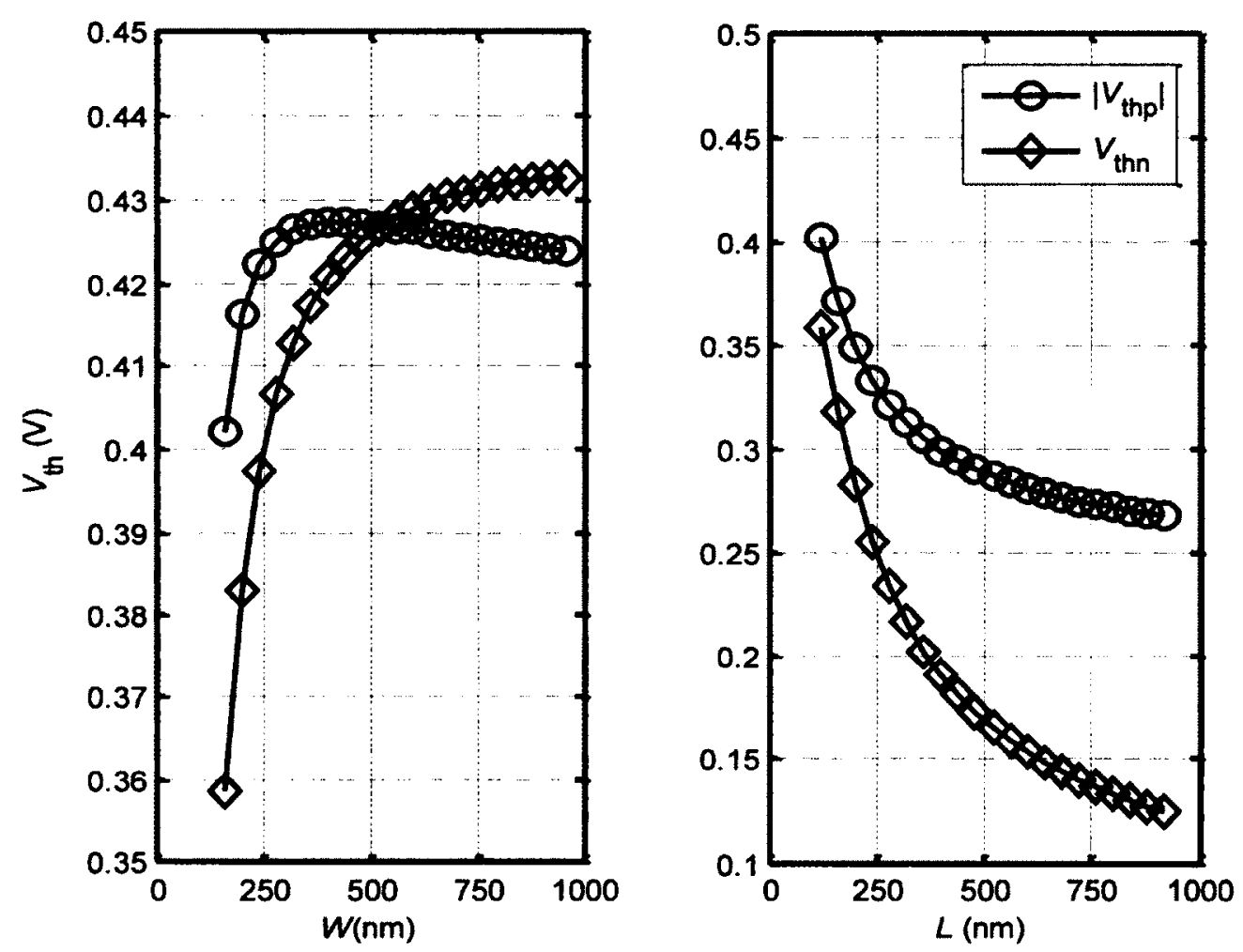

Figure 4.1 Threshold voltage versus the channel width at $L_{\min }=120 \mathrm{~nm}$ (left), and threshold voltage versus the channel length at $W_{\min }=160 \mathrm{~nm}$ (right) for IBM $130 \mathrm{~nm}$ technology.

Figure 4.2 and Figure 4.3 show the PMOS and NMOS transistors' threshold voltage variation with respect to the channel width in different technology nodes, respectively. As it is appearing in Figure 4.2, INWE is not significant for PMOS transistors in the $65 \mathrm{~nm}$ technology. It means that for $65 \mathrm{~nm}$ PMOS transistors, the sub-threshold current shows a nearly linear relation to $W$, as shown in Figure 4.9 (d,e) of Section 4.2.

Figure 4.4 and Figure 4.5 show variation of the threshold voltage with respect to the channel length for the PMOS and NMOS transistors, respectively. Due to the RCSE, the threshold voltages show roll-up as the channel length decreases in all considered technologies. 


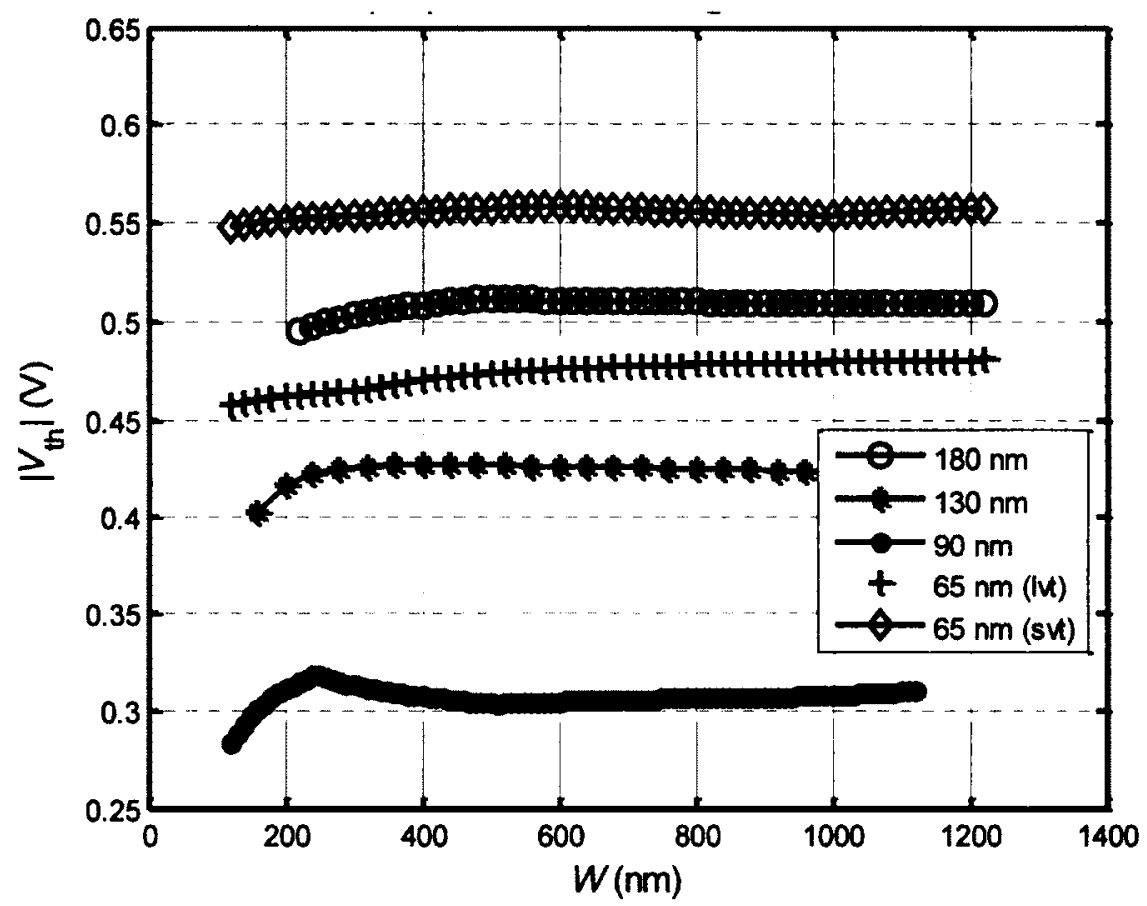

Figure 4.2 Threshold voltage versus $W$ at $V_{\mathrm{DD}}=0.2 \mathrm{~V}$ for a PMOS transistor in different technology nodes at $L=L_{\min }$

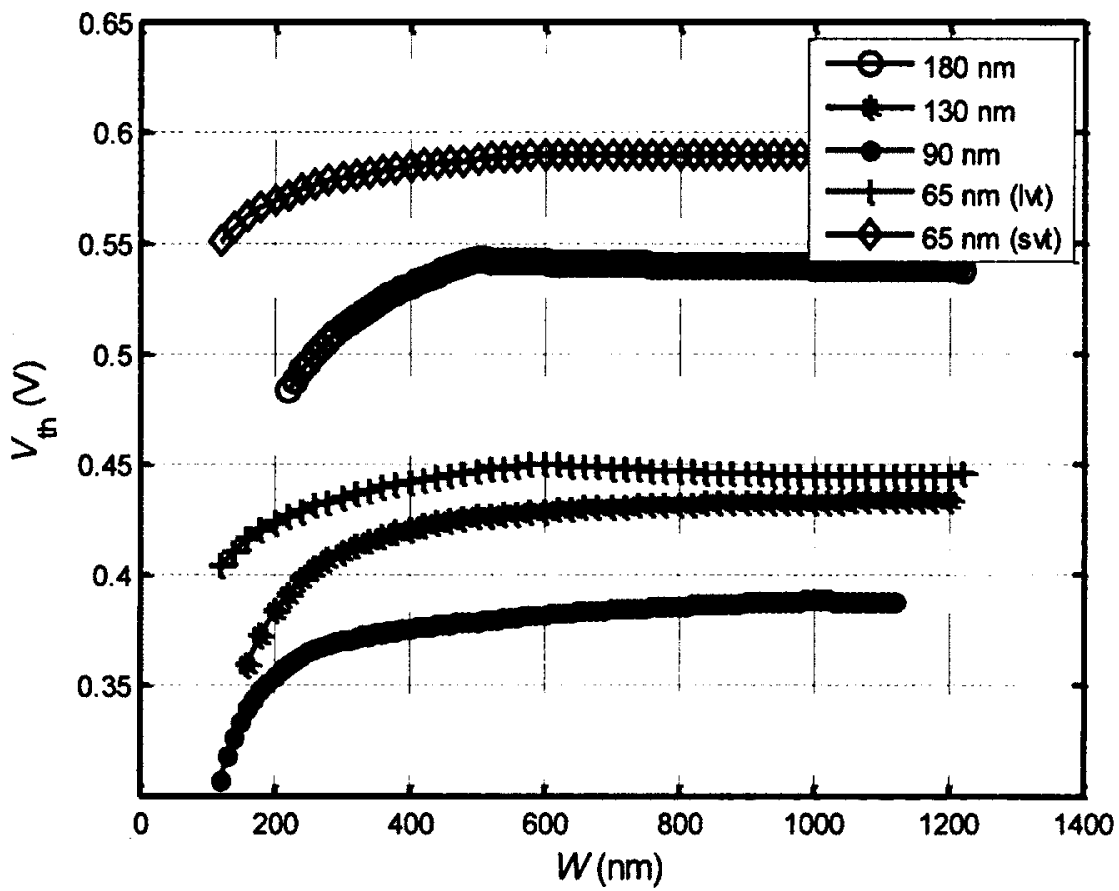

Figure 4.3 Threshold voltage versus $W$ at $V_{\mathrm{DD}}=0.2 \mathrm{~V}$ for an NMOS transistor in different technology nodes at $L=L_{\min }$ 


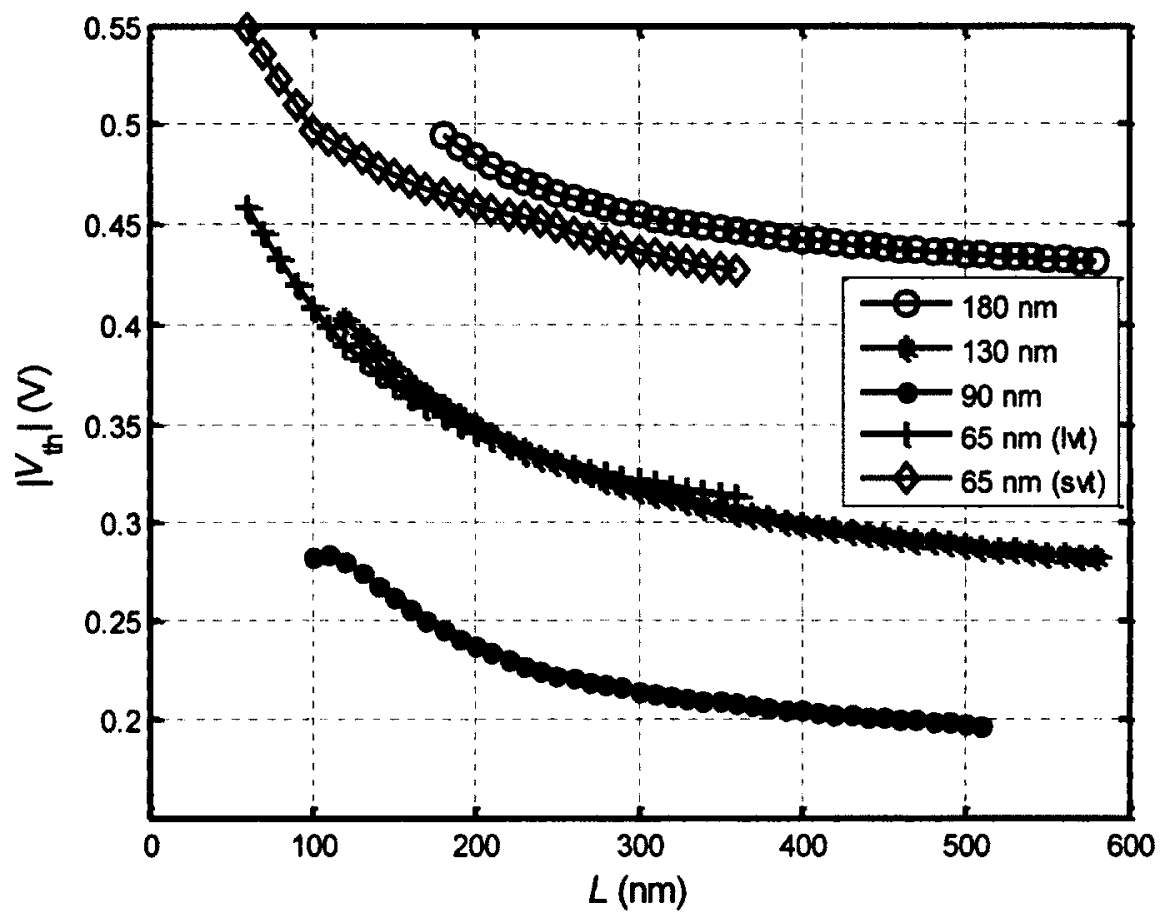

Figure 4.4 Threshold voltage versus $L$ at $V_{\mathrm{DD}}=0.2 \mathrm{~V}$ for a PMOS transistor in different technology nodes at $W=W_{\min }$

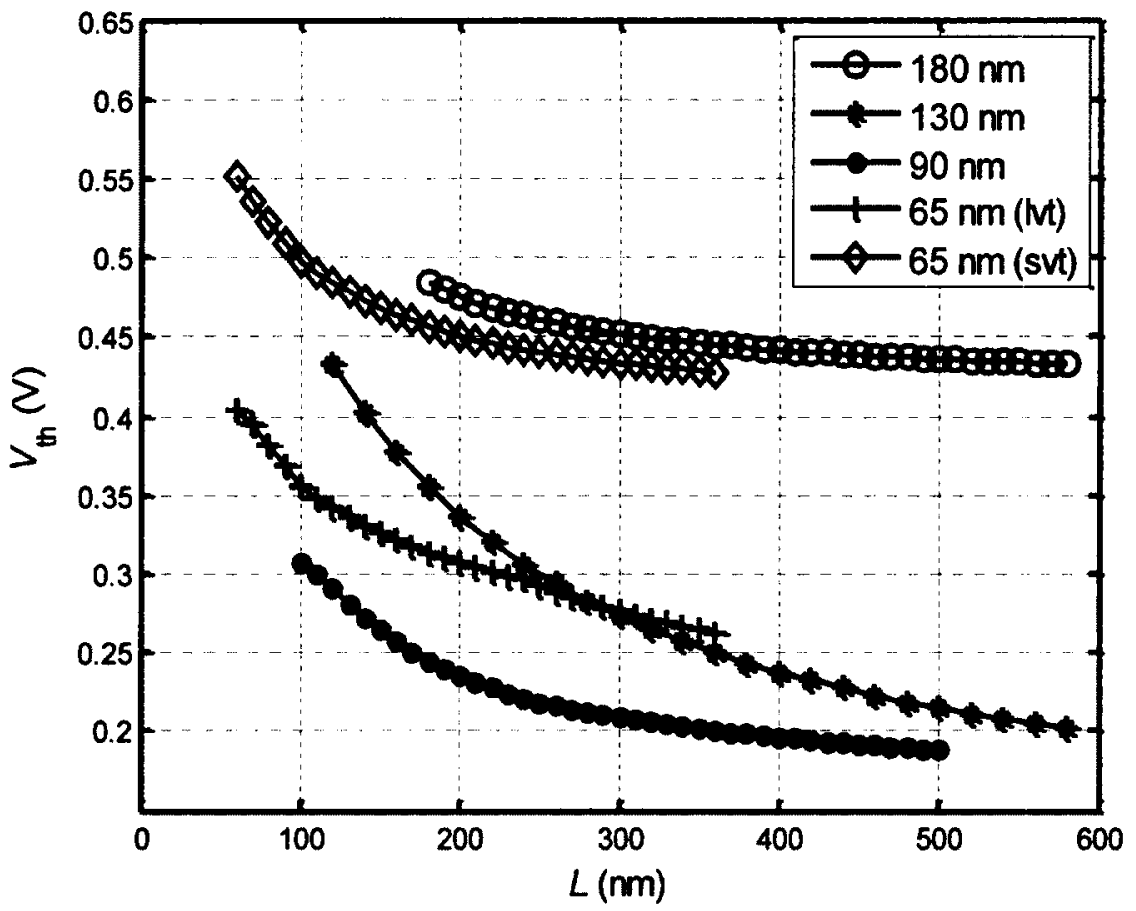

Figure 4.5 Threshold voltage versus $L$ at $V_{\mathrm{DD}}=0.2 \mathrm{~V}$ for an NMOS transistor in different technology nodes at $W=W_{\min }$ 


\subsection{Current Behaviour}

According to the super-threshold current Equation (3-1), due to the nearly linear relation between the current and the threshold voltage, a small change in the threshold voltage does not show significant any influence on the current. Therefore, the current is a nearly linear ascending function of $W$ and a descending function of $L$ as depicted in Figure 4.6.

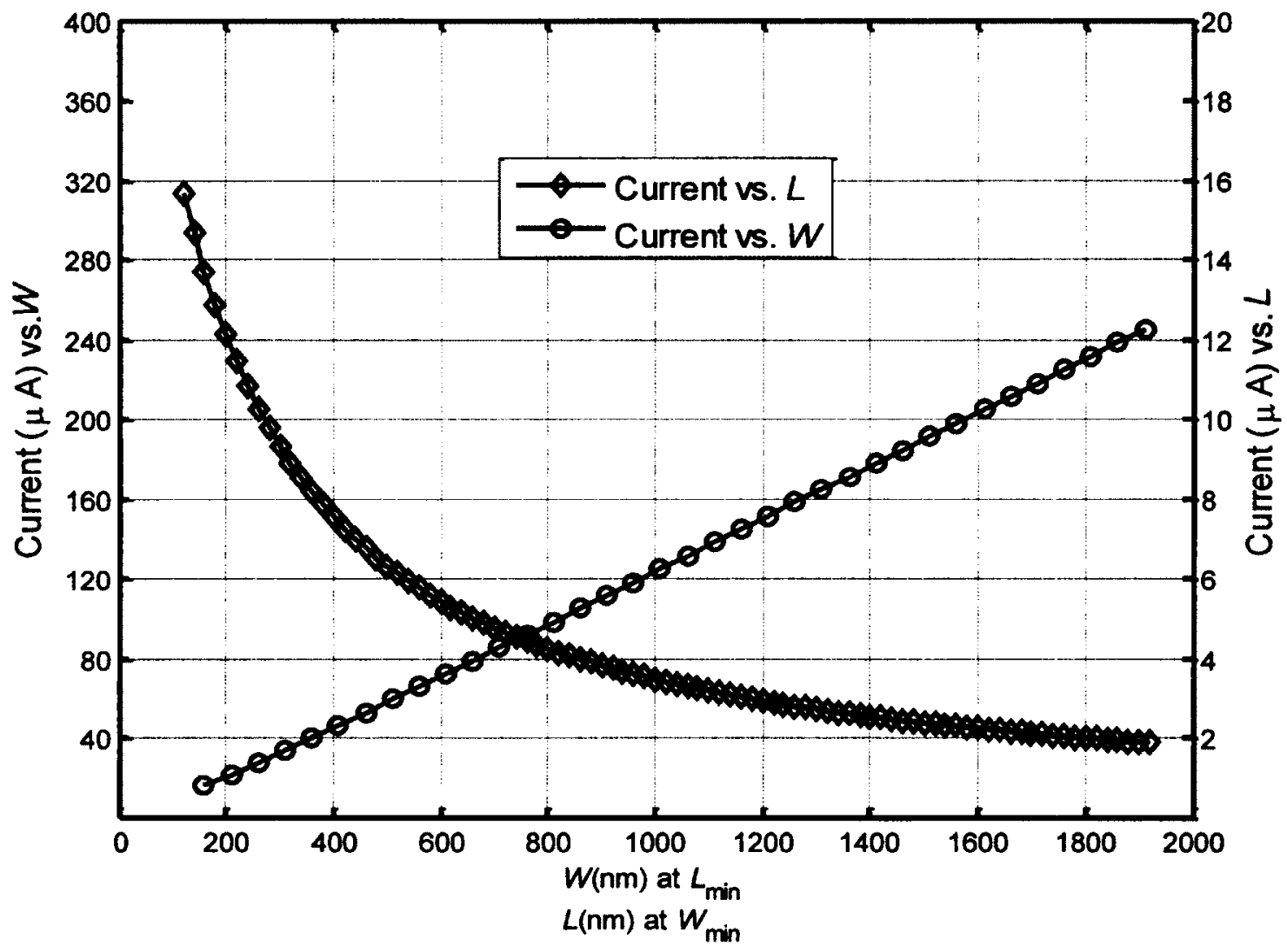

Figure 4.6 Super-threshold current for a PMOS transistor at $V_{D D}=1 \mathrm{~V}$ versus $L$ and $W$, in IBM 130nm CMOS (NMOS transistor shows the same behavior).

However, in the sub-threshold region, because of the exponential dependence of the current to the threshold voltage, the behavior of the current is not as simple as the superthreshold current. Quoting Equation (2-3), factors $W / L$ and $\exp \left(\frac{V_{G S}-V_{t h}}{n V_{T}}\right)$ do not show the same behavior (with respect to each other) as the transistor dimensions change.

For instance, when $L$ increases (decreases), $V_{t h}$ decreases (increases) as shown in Figure 4.4 and Figure 4.5. Thus, $1 / L$ decreases (increases) while $\exp \left(\frac{V_{G S}-V_{t h}}{n V_{T}}\right)$ increases 
(decreases). Since the sub-threshold current is proportional to the multiplication of these two last factors, the current shows an unpredictable behavior with respect to the channel length variations. Depending on which factor changes more, the current tracks its behavior. For example, if we double the channel length, $W / L$ will be halved, while the amount of change in $\exp \left(\frac{V_{G S}-V_{t h}}{n V_{T}}\right)$ is unknown. Suppose that $\exp \left(\frac{V_{G S}-V_{t h}}{n V_{T}}\right)$ becomes doubled. Hence, the multiplication of these two factors does not change; i.e., no change in the current occurs. However, if $\exp \left(\frac{V_{G S}-V_{t h}}{n V_{T}}\right)$ increases more (less) than twice, the current shows an increase (decrease). Thus, the current could be an ascending or a descending function of $L$ and the same discussion is valid for $W$.

In Figure 4.7 the two factors are plotted in the top figure for a PMOS-lvt transistor in the TSMC $65 \mathrm{~nm}$ LP CMOS technology. It is depicted that these two factors vary in opposite directions with respect to the channel length. Multiplication of these two factors
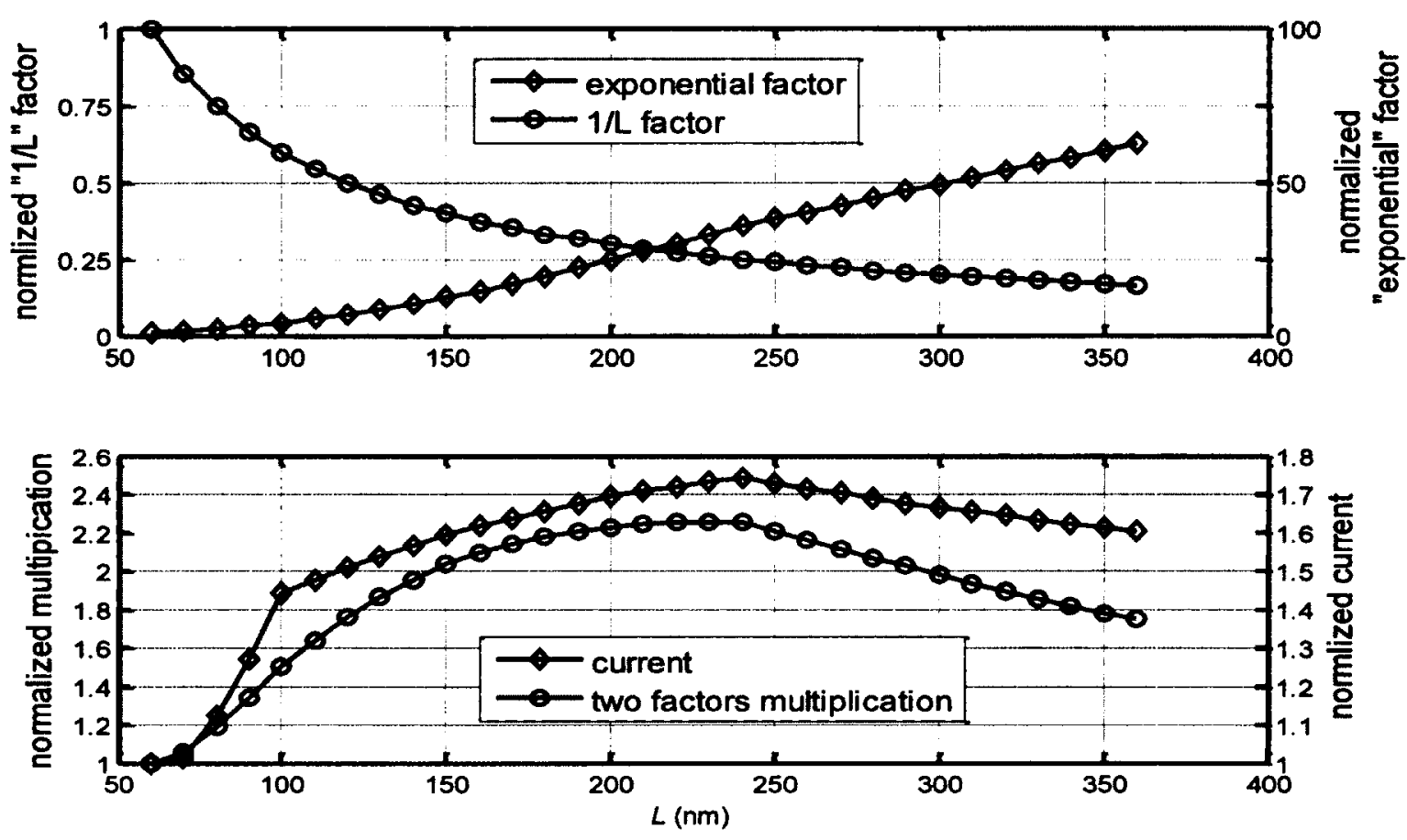

Figure 4.7 Top figure shows normalized $1 / L$ and $\exp \left(\frac{V_{G S}-V_{t h}}{n V_{T}}\right)$ factors individually plotted versus $L$. The bottom figure shows normalized $1 / L \times \exp \left(\frac{V_{G S}-V_{t h}}{n V_{T}}\right)$ and normalized current at $V_{D D}=0.2$ V for TSMC $65 \mathrm{~nm}$ LP CMOS kit for a PMOS-lvt transistor at $W=W_{\mathrm{m} / \mathrm{n}}=120 \mathrm{~nm}$. 
is plotted in the bottom figure. It shows a peak at the same point, where the actual current shows its maximum point.

Figure 4.8 shows $1 / L$ and $\exp \left(\frac{V_{G S}-V_{t h}}{n V_{T}}\right)$ factors for a PMOS-svt in the TSMC $65 \mathrm{~nm}$ LP CMOS (top figure). Although the exponential factor shows an incremental behaviour with respect to $L$, like what occurs for a PMOS-lvt, the multiplication of these two factors does not show any maximum value contrary to what occurs for a PMOS-lvt. This fact shows that despite of ascending behaviours of the exponential factor with respect to $L$ in both PMOS transistors, the product of $1 / L$ and $\exp \left(\frac{V_{G s}-V_{t h}}{n V_{T}}\right)$, which replicates the subthreshold current, might have different behaviours versus $L$.
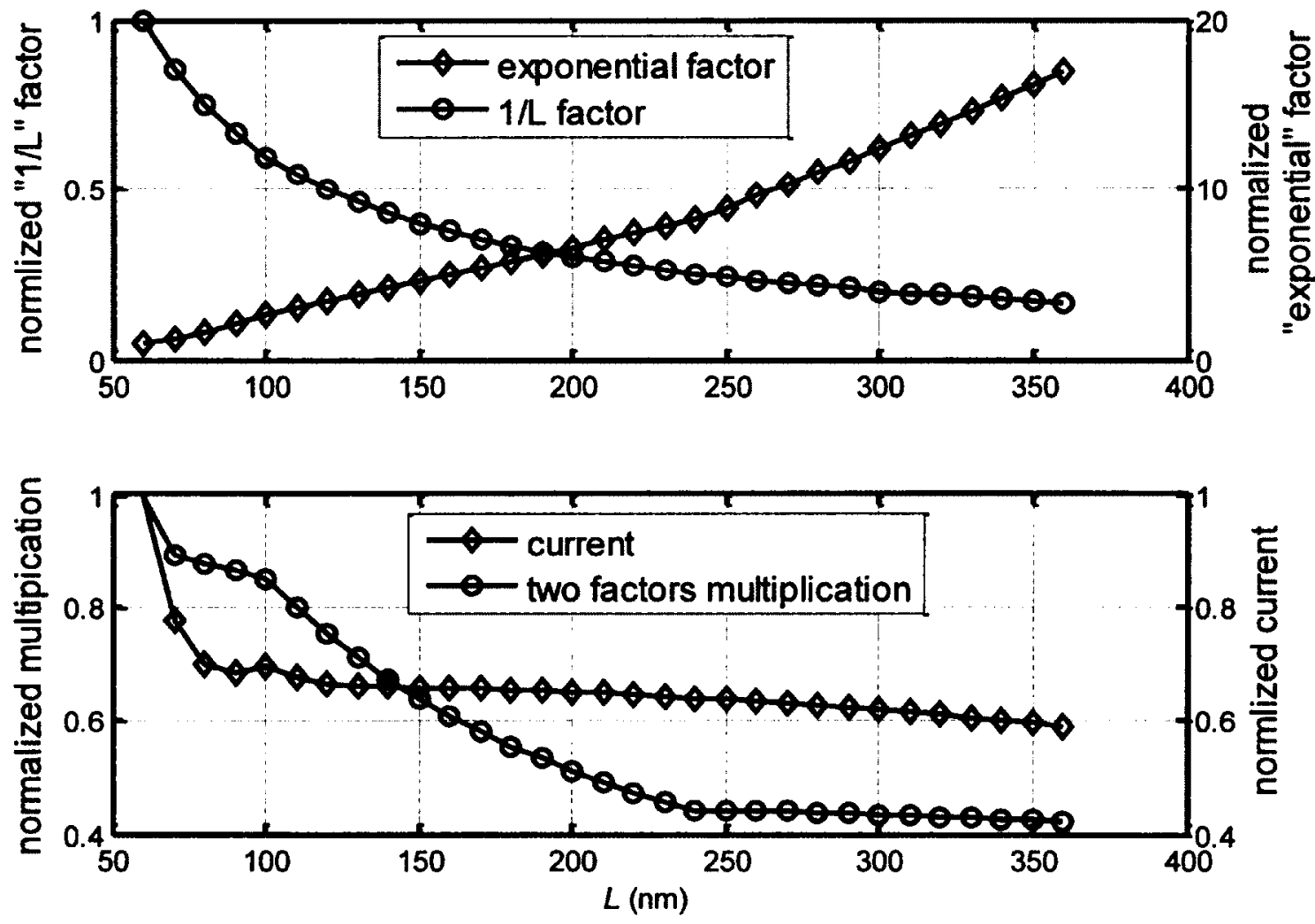

Figure 4.8 Top figure shows normalized $1 / L$ and $\exp \left(\frac{V_{G S}-V_{\text {th }}}{n V_{T}}\right)$ factors individually plotted versus $L$. The bottom figure shows normalized $1 / L \times \exp \left(\frac{V_{G s}-V_{t h}}{n V_{T}}\right)$ and normalized current at $V_{D D}=0.2$ V for TSMC $65 \mathrm{~nm}$ LP CMOS kit for a PMOS-svt transistor at $W=W_{\min }=120 \mathrm{~nm}$. 
The current behavior in the sub-threshold region with respect to $W$ and $L$ not only varies from one technology node to another technology node, but also varies from one transistor type to another transistor type in the same technology node. Moreover, in some technology nodes where different flavours of transistors are provided, for example lvt and svt in the TSMC $65 \mathrm{~nm} \mathrm{LP} \mathrm{CMOS,} \mathrm{the} \mathrm{same} \mathrm{type} \mathrm{transistors} \mathrm{might} \mathrm{show} \mathrm{different}$ behavior.

As a summary, the sub-threshold current versus $W$ and $L$ are shown in Figure 4.9 and Figure 4.10, respectively. As it is appearing in these figures, and consistent with what discussed before, the current behaviour is not the same for all technologies.

$180 \mathrm{~nm}$

(a)

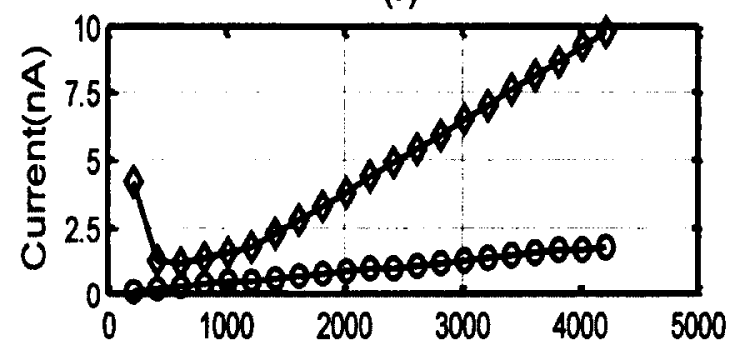

$90 \mathrm{~nm}$

(c)

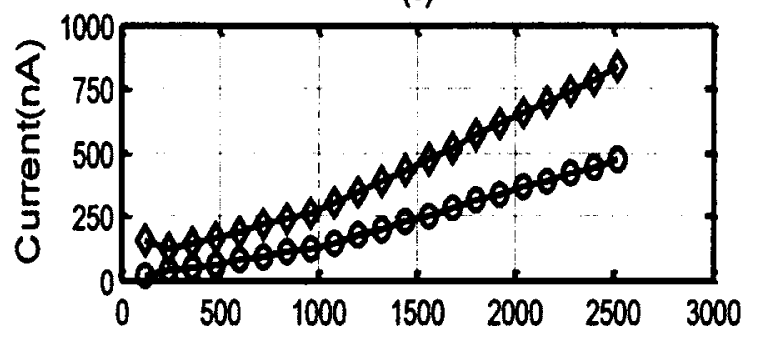

$65 \mathrm{~nm}$ LP (svt transistors)

(e)

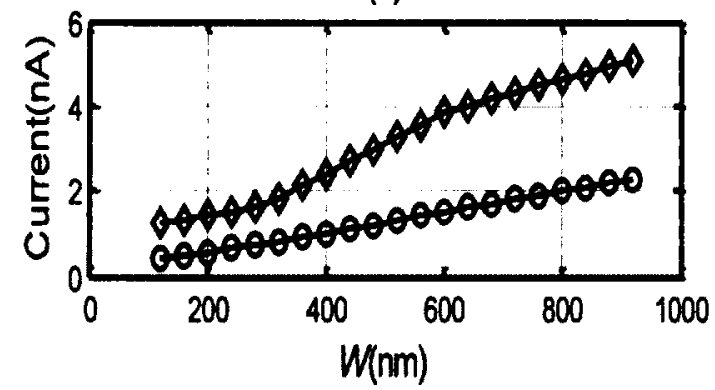

$130 \mathrm{~nm}$

(b)

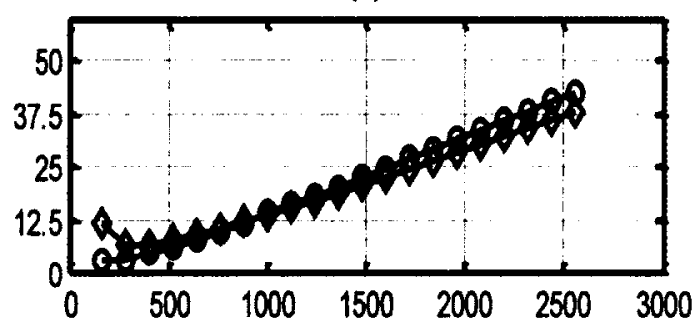

$65 \mathrm{~nm}$ (Wt transistors)

(d)

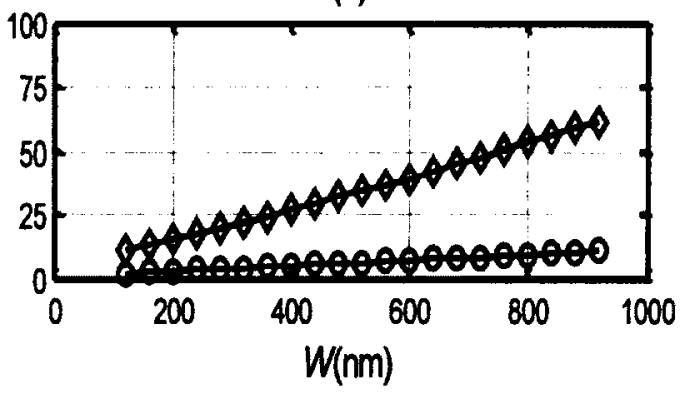

Figure 4.9 Sub-threshold current versus $W$ in different technology nodes at $V_{\mathrm{DD}}=0.2 \mathrm{~V}$ and $L_{\text {min }}$. 
Figure 4.11 shows that in the $130 \mathrm{~nm}, 90 \mathrm{~nm}$, and $65 \mathrm{~nm}$ (lvt) technology kits, both NMOS and PMOS transistors show a peak value versus $L$ (Figure $4.11(\mathrm{~b}, \mathrm{c}, \mathrm{d})$ ), while in the $180 \mathrm{~nm}$ only the PMOS transistor has a maximum point versus $L$ (Figure 4.11(a)).
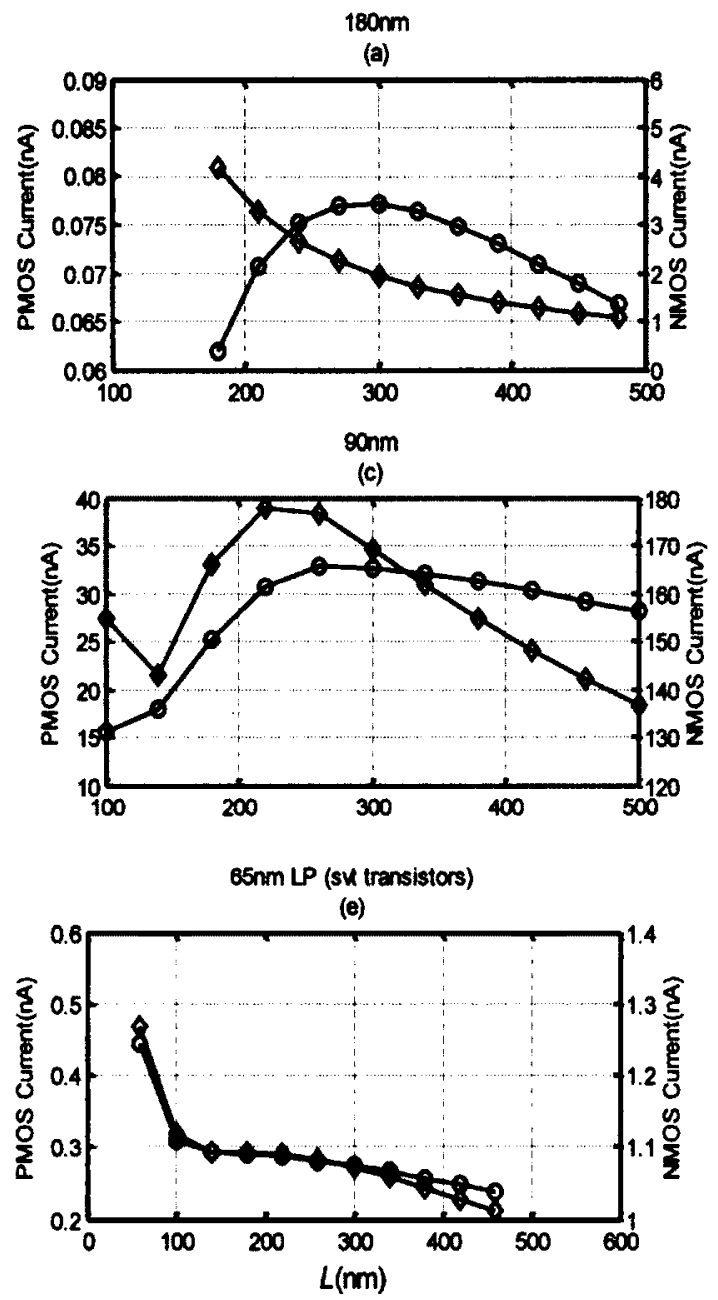
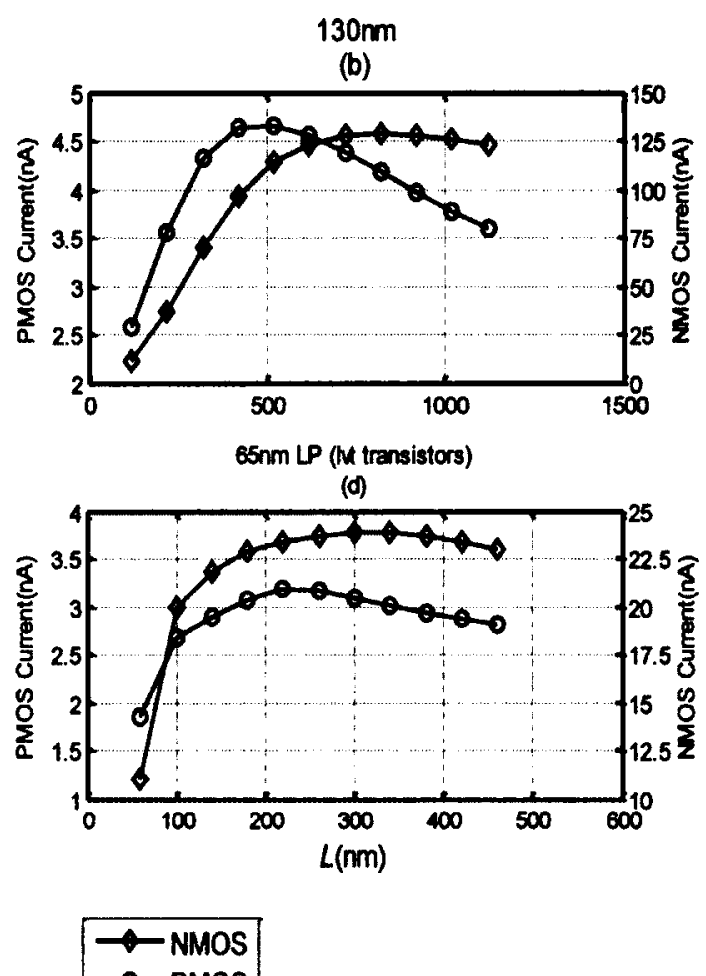

Figure 4.10 Sub-threshold current versus $L$ in different technology nodes at $V_{\mathrm{DD}}=0.2 \mathrm{~V}$ and $W_{\min }$.

One should notice that the maximum point for the sub-threshold current versus the channel length depends on the supply voltage. As the supply voltage increases, the maximum point for the current becomes closer to the minimum length. Figure 4.11 shows that in the $130 \mathrm{~nm}$ technology for larger supply voltages, the maximum point for the current occurs in smaller lengths. This also happens in the other technologies. Figure 4.12 shows the channel length where the sub-threshold current becomes maximum, $L_{\operatorname{lmax}}$, versus the supply voltage for different CMOS technologies. As the supply voltages reach 
the super-threshold region, $L_{\mathrm{Imax}}$ moves to the minimum length. In different technologies this happens in different supply voltages. According to Figure 4.4 and Figure 4.5, the threshold voltage in the $90 \mathrm{~nm}$ technology is the smallest threshold voltage among the four considered technologies. Hence, it is predictable that $L_{\mathrm{Imax}}$ for the $90 \mathrm{~nm}$ becomes the minimum channel length in smaller supply voltages, which is verified in Figure 4.12.

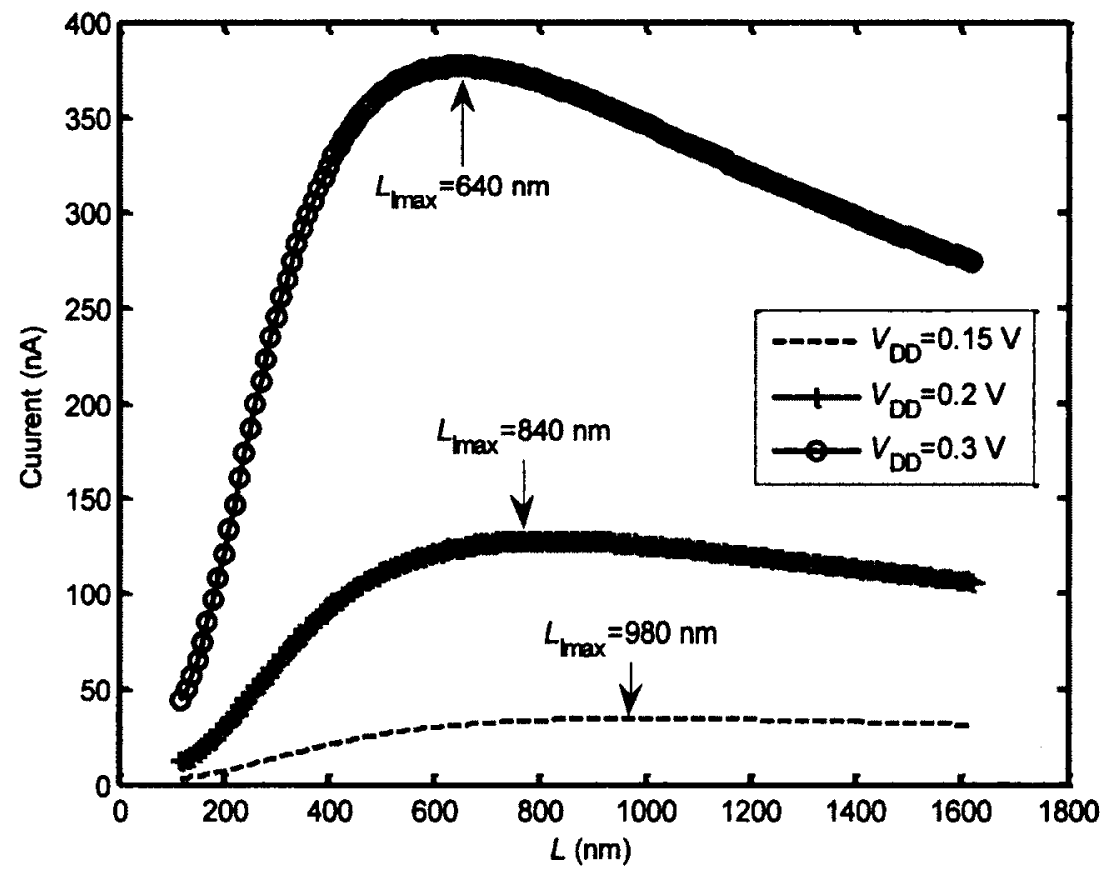

Figure 4.11 Sub-threshold current versus the channel length for an NMOS transistor in IBM $130 \mathrm{~nm}$ technology at $W_{\min }$. The maximum point becomes smaller as the supply voltage increases.

\subsection{MOSFET Capacitances}

Another important element that should be studied for the delay and energy optimization is the transistor capacitances. As discussed in Section 3.1.3, there are several different types of capacitances between the transistors terminals. In that section we just introduced the general concept, but in this section we will study the behaviour of all capacitances in more detail with respect to the transistor dimensions and its terminal voltages. 

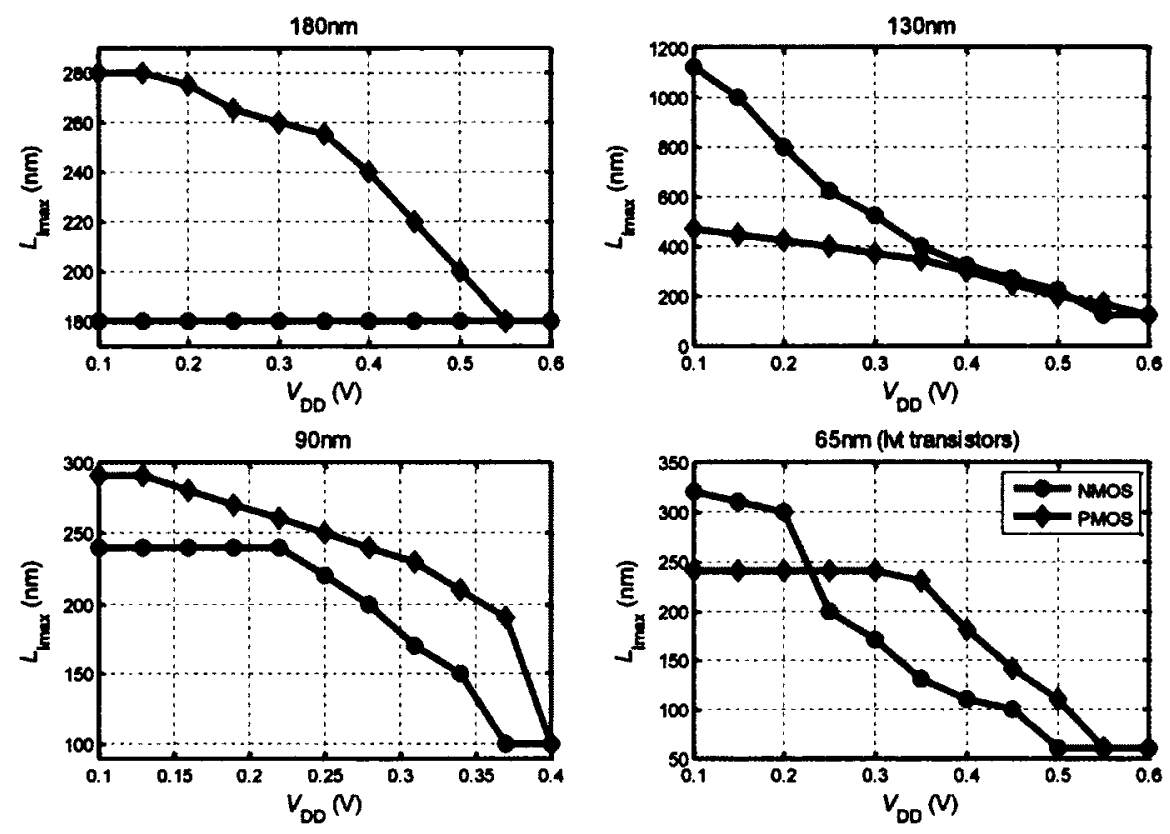

Figure 4.12 $L_{I \max }$ versus $V_{D D}$ at $W_{\text {min }}$.

\section{Voltage dependence of MOSFET capacitances}

As explained in Section 3.1.3, the gate capacitance of a MOSFET is a combination of the gate oxide capacitance, fringing capacitance due to the gate overlap to source and drain, and depletion capacitance under the gate area. Among all these mentioned capacitances, only the first portion of the gate capacitance is independent of the gate voltage, but the rest are non-linear functions of the gate voltage. For example, Figure 4.13 shows the dependence of the gate capacitances to the gate voltage. Simulations are performed for the NMOS transistor in the IBM $130 \mathrm{~nm}$ technology for two different values of $V_{\mathrm{DS}}$. In small $V_{\mathrm{DS}}$ where the channel is uniform, $C_{\mathrm{GD}}$ and $C_{\mathrm{GS}}$ change in the same manner. At higher $V_{\mathrm{DS}}$ where the channel is narrower near the drain, $C_{\mathrm{GS}}$ is bigger than $C_{\mathrm{GD}}$. The capacitance $C_{\mathrm{GG}}$ is $C_{\mathrm{GD}}+C_{\mathrm{GS}}+C_{\mathrm{GB}}$ and is almost independent of $V_{\mathrm{DS}}$, but is a nonlinear function of the gate voltage. In delay optimization, to have a more accurate result, one should take this behaviour into account.

There are two other capacitances that are involved in the delay and energy calculation and optimization. These capacitances are the capacitances between the source and drain junctions and the body due to the reverse- biased diodes at these junctions. As evident 

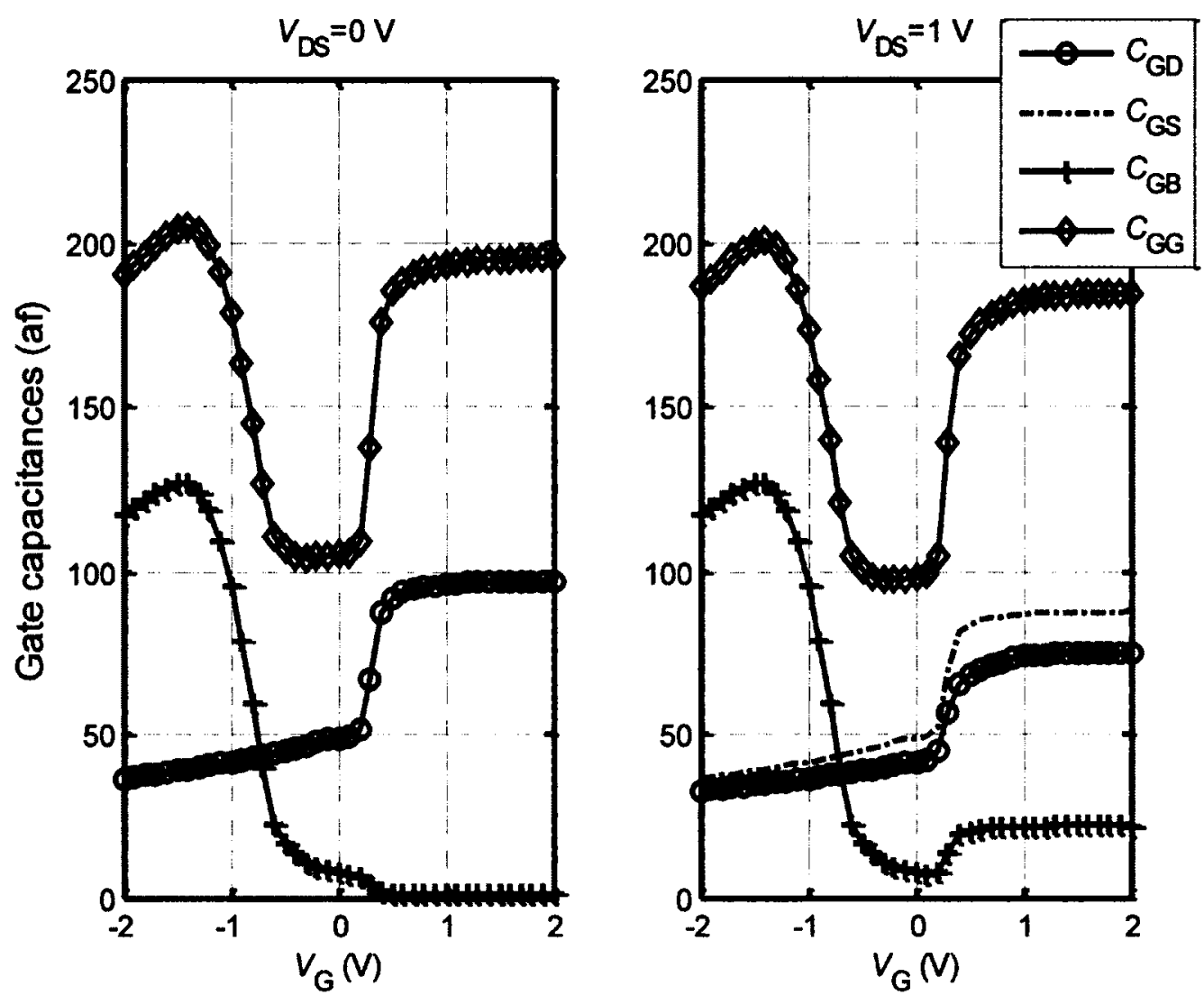

Figure 4.13 Gate capacitances versus gate voltage for an NMOS transistor in IBM $130 \mathrm{~nm}$ for two different $V_{\mathrm{DS}} . C_{\mathrm{GG}}$ is equal to the sum of the other three capacitances.

from Equation (3-12), these capacitances are non-linear functions of the reverse-bias voltages applied to these junctions. If the source and body are connected together, like the case of an inverter, only $C_{\mathrm{DB}}$ affects the delay and energy. In Figure 4.14 the drain-body capacitances for an NMOS transistor in IBM $130 \mathrm{~nm}$ technology is plotted as an example. It shows the non-linear relation of this capacitance to $V_{\mathrm{DS}}$ and also shows its independence of $V_{\mathrm{GS}}$. In other technologies the same behaviour is seen, but here only the results of the IBM $130 \mathrm{~nm}$ are reported.

\section{Size dependence of MOSFET capacitances}

The capacitances of a MOSFET are related to both the channel length and width, except for the two junction capacitances that are only related to the channel width. In this section we will study the effect of the channel length and width only on $C_{\mathrm{GG}}$ and $C_{\mathrm{DB}}$ in the sub-threshold region. As depicted in Figure 4.15, both capacitances are linear function of the channel width. However, the gate capacitance is not a linear function of the channel length near the minimum channel length. The drain junction capacitance 
remains constant regardless of the changes in the channel length. The total capacitance, $C_{\text {total, }}$, that affects the delay, is the sum of these two capacitances. $C_{\text {total }}$ is a linear function of the channel width and almost a linear function of the channel length despite $C_{\mathrm{DB}}$ 's independence of $L$.

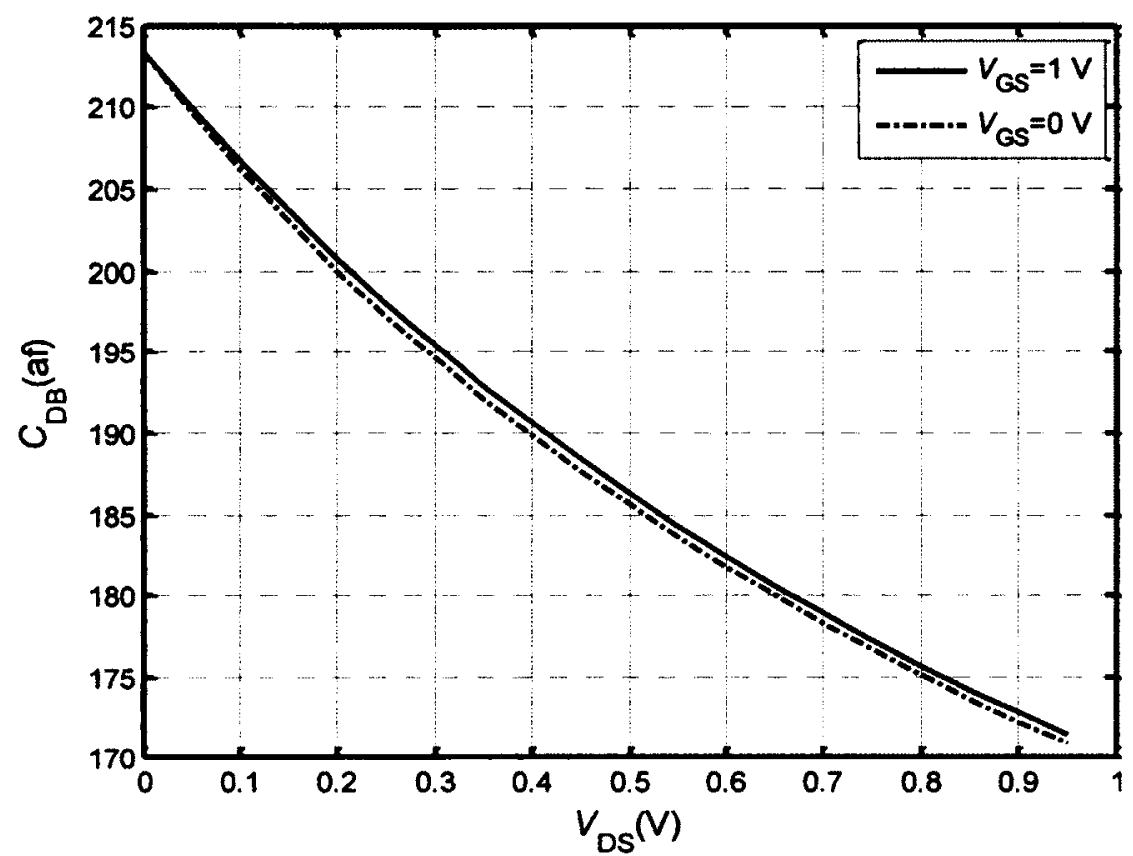

Figure 4.14 Drain-body junction capacitance versus $V_{D S}$ for an NMOS in IBM $130 \mathrm{~nm}$ technology at two different $V_{\text {GS. }}$.

In calculating the propagation delay, as described in Section 3.2.1, one should use an effective value for the capacitances involved in the delay. In the sub-threshold region, since the voltage variation is not large, it seems that there isn't much of a variation in $C_{\text {total. }}$ This approximation is only valid with respect to the channel width changes. However, when the channel length changes, the dependence of $C_{\text {total }}$ to the voltage is more significant. When the channel length is small, HALO doping areas merge together and make the surface doping level higher. Vice versa, when the channel length becomes longer, HALO regions becomes further separated and the surface doping falls. Since the depletion capacitance under the gate is a function of the surface doping, this capacitance shows more variations with respect to $L$ rather than $W$. As a consequence, $C_{\text {total }}$ shows more sensitivity to voltage variations with respect to $L$ than $W$. Figure 4.16 verifies that $C_{\text {total }}$ shows more dependence to the voltage when the channel length is changing. 

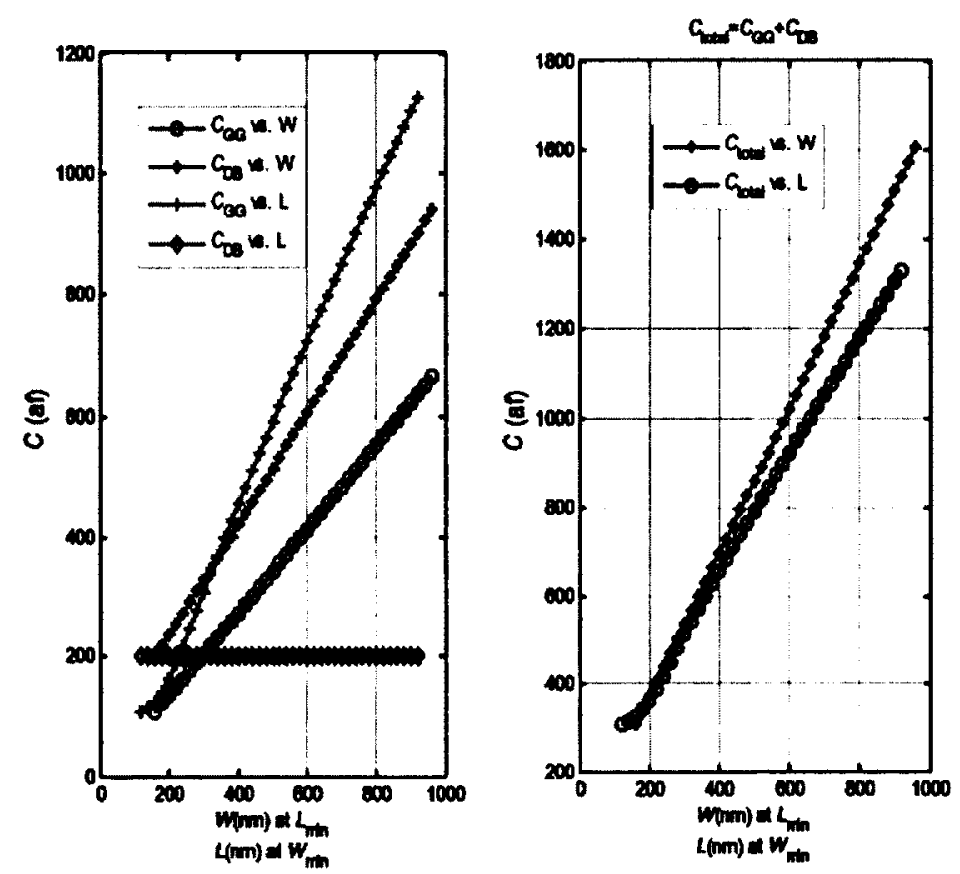

Figure 4.15 Gate and drain capacitances versus the channel width and channel length for an NMOS in IBM $130 \mathrm{~nm}$ at $V_{\mathrm{DS}}=V_{\mathrm{GS}}=0.2 \mathrm{~V}$.
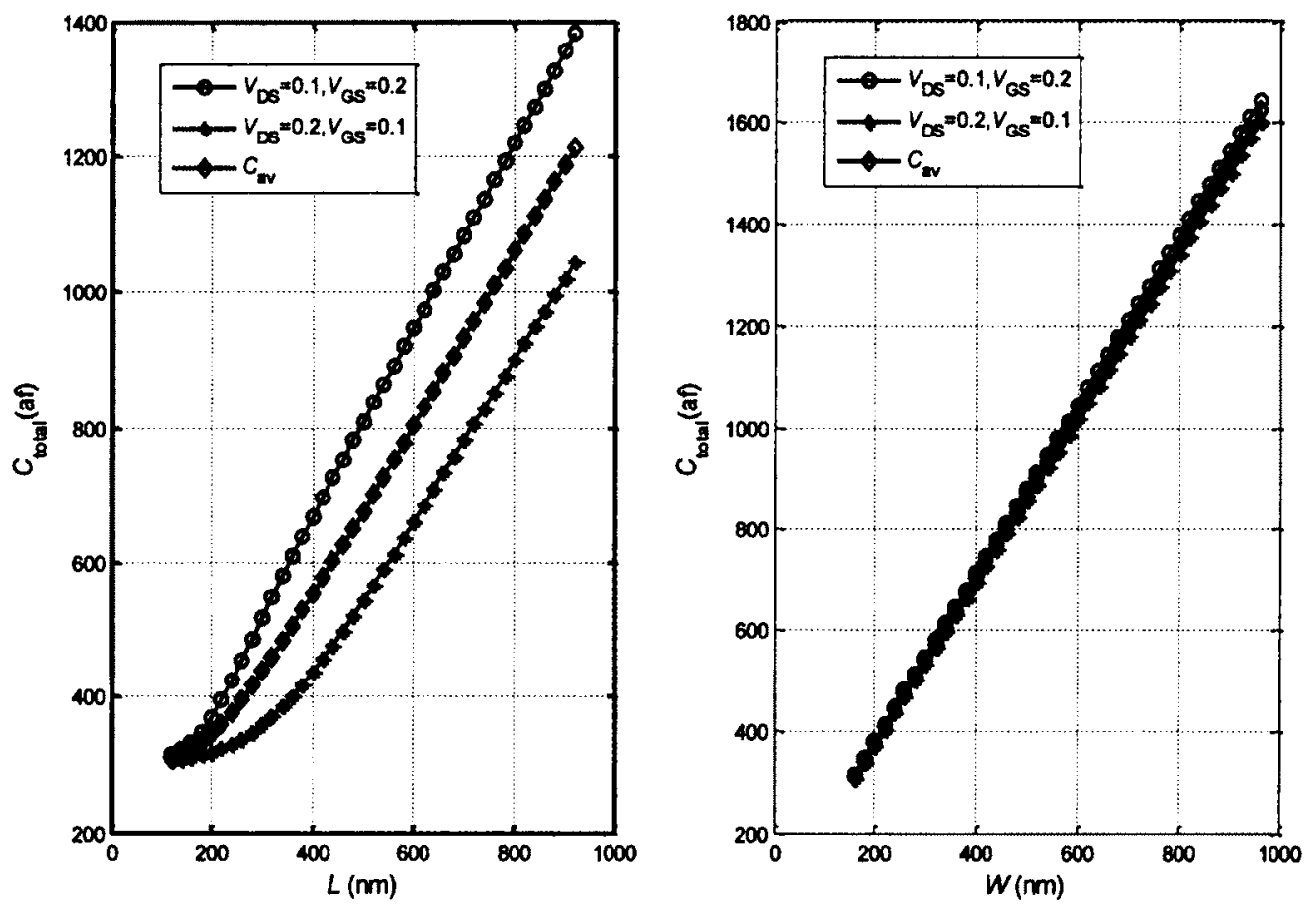

Figure 4.16 $C_{\text {total }}$ versus the channel length (left) and versus the channel width (right) for an NMOS in IBM $130 \mathrm{~nm}$ for two sets of voltages that are used in delay modeling and estimation. 


\subsection{Leakage Currents}

Understanding the leakage currents' behaviour is the main key to optimize the energy consumption, especially in the sub-threshold region where the relative contribution of the leakage energy is more than the case of the super-threshold region. In this section we will study the effect of transistor sizing on three main components of the leakage current: the gate leakage $\left(I_{\mathrm{G}}\right)$, the sub-threshold leakage $\left(I_{\mathrm{off}}\right)$, and the source/drain junctions' leakage ( $\left.I_{\text {Bulk }}\right)$. The general concepts of these leakage currents were presented in Section 3.1.4. To measure the leakage currents, the simulation test benches are arranged as shown in Figure 4.17.

The simulation measurement results for the two types transistor are shown in Table 4.1. As can be seen in this table, the sub-threshold leakage, $I_{\text {off, }}$ has the biggest portion among the leakage currents elements. This element of leakage current is about three orders of magnitude larger than the two other leakage elements. Changing the transistor dimensions doesn't lead to a significant change on this percentage and $I_{\text {off }}$ remains as the dominant leakage element regardless of the transistor dimensions. Therefore, in the following discussion our main focus is on $I_{\text {off }}$ as the main leakage current component.

In the sub-threshold region of operation both $I_{\text {on }}$ and $I_{\text {off }}$ are expressed by Equation (3-3) except that to calculate $I_{\mathrm{off}}$, the gate-source voltage $V_{\mathrm{GS}}$ is set to " 0 ". Hence, it seems that the "off" and "on" currents show the same behaviour with respect to the changes in the transistor size. For instance, in Figure 4.18 the "off" and "on" currents for a PMOS transistor in IBM $130 \mathrm{~nm}$ are illustrated at $V_{\mathrm{DD}}=0.2 \mathrm{~V}$. As seen in the graphs, $I_{\text {off }}$ and $I_{\text {on }}$ have very similar changes with respect to the channel length and width variations. A minor difference can be noticed when the channel length is changing. This difference is due to the dependence of the charge carries mobility $(\mu)$ and the subthreshold slope factor $(n)$ to the gate-source voltage ${ }^{l}$. These dependences to $V_{\mathrm{GS}}$ are more noticeable for the channel length variation than the channel width variation. Although not reported here, we found the same trend to be true for other technologies.

\footnotetext{
${ }^{1} V_{\mathrm{GS}}$ affects $\mu$ due to the mobility degradation [15] and affects $n$ due to the induced changes in the
} depletion region under the gate. 
Table 4.1 The leakage currents for NMOS and PMOS transistors in each technology at their minimum acceptable sizes.

\begin{tabular}{|c|c|c|c|c|c|c|c|c|}
\hline & \multicolumn{2}{|c|}{$I_{G}$} & \multicolumn{2}{|c|}{ Joff } & \multicolumn{2}{|c|}{ I Bulk } \\
\hline & & & $\begin{array}{l}\text { Super-threshold } \\
\qquad V_{\mathrm{DD}}=1\end{array}$ & $\begin{array}{c}\text { Sub-threshold } \\
V_{\mathrm{DD}}=0.2\end{array}$ & $\begin{array}{l}\text { Super-threshold } \\
\qquad V_{\mathrm{DD}}=1\end{array}$ & $\begin{array}{c}\text { Sub-threshold } \\
V_{\mathrm{DD}}=0.2\end{array}$ & $\begin{array}{l}\text { Super-threshold } \\
\qquad V_{\mathrm{DD}}=1\end{array}$ & $\begin{array}{c}\text { Sub-threshold } \\
V_{\mathrm{DD}}=0.2\end{array}$ \\
\hline \multirow{2}{*}{\multicolumn{2}{|c|}{$180 \mathrm{~nm}$}} & NMOS & 0 & 0 & $552 \mathrm{f}$ & $15.32 p$ & $5.76 \mathrm{a}$ & $5.76 \mathrm{a}$ \\
\hline & & PMOS & 0 & 0 & $26.48 p$ & $172.2 \mathrm{f}$ & $4.75 \mathrm{a}$ & $4.75 a$ \\
\hline \multirow{2}{*}{\multicolumn{2}{|c|}{$130 \mathrm{~nm}$}} & NMOS & $4.14 f$ & $70.57 \mathrm{a}$ & $217.3 p$ & $59.61 \mathrm{p}$ & $639 a$ & $156 \mathrm{z}$ \\
\hline & & PMOS & $819 a$ & $18.45 \mathrm{a}$ & $39.47 p$ & $14.53 p$ & $2.1 \mathrm{a}$ & $64.39 \mathrm{z}$ \\
\hline \multirow{2}{*}{\multicolumn{2}{|c|}{$90 \mathrm{~nm}$}} & NMOS & $30.6 \mathrm{p}$ & $810 \mathrm{f}$ & $2.966 n$ & $952 p$ & $9.67 \mathrm{f}$ & $1.97 \mathrm{a}$ \\
\hline & & PMOS & $12.11 p$ & $486 f$ & $217.4 p$ & $64.19 p$ & $44.8 \mathrm{a}$ & $838 z$ \\
\hline \multirow{4}{*}{$\begin{array}{l}\stackrel{\mathscr{B}}{\Xi} \\
\stackrel{\Xi}{\Xi}\end{array}$} & \multirow{2}{*}{ lvt } & NMOS & $469 f$ & $8.62 \mathrm{f}$ & $201 p$ & $25.98 p$ & $389 a$ & 3202 \\
\hline & & PMOS & $218 \mathrm{f}$ & $5.58 \mathrm{f}$ & $53 p$ & $7.46 \mathrm{p}$ & $92 a$ & $195 \mathrm{z}$ \\
\hline & \multirow{2}{*}{ svt } & NMOS & $137 \mathrm{f}$ & $3.412 f$ & $16.06 \mathrm{p}$ & $4.162 p$ & $49 a$ & $348 z$ \\
\hline & & PMOS & $163 \mathrm{f}$ & $3.4 \mathrm{f}$ & $8.865 p$ & $2.23 p$ & $88 a$ & $227 z$ \\
\hline
\end{tabular}

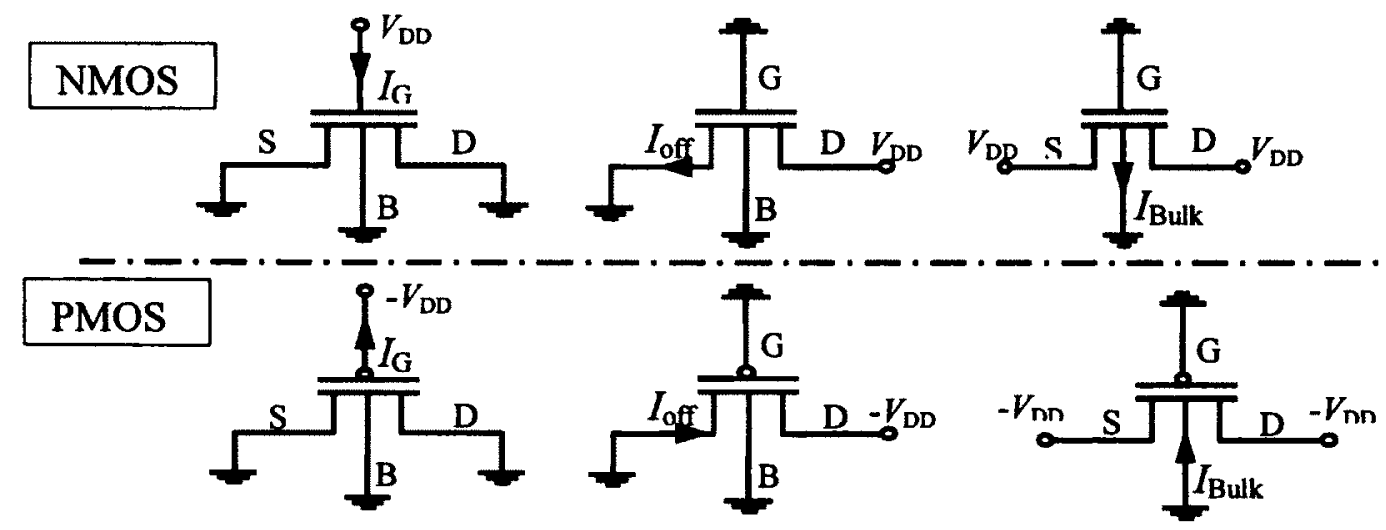

Figure 4.17 Test benches used for leakage currents measurement. 


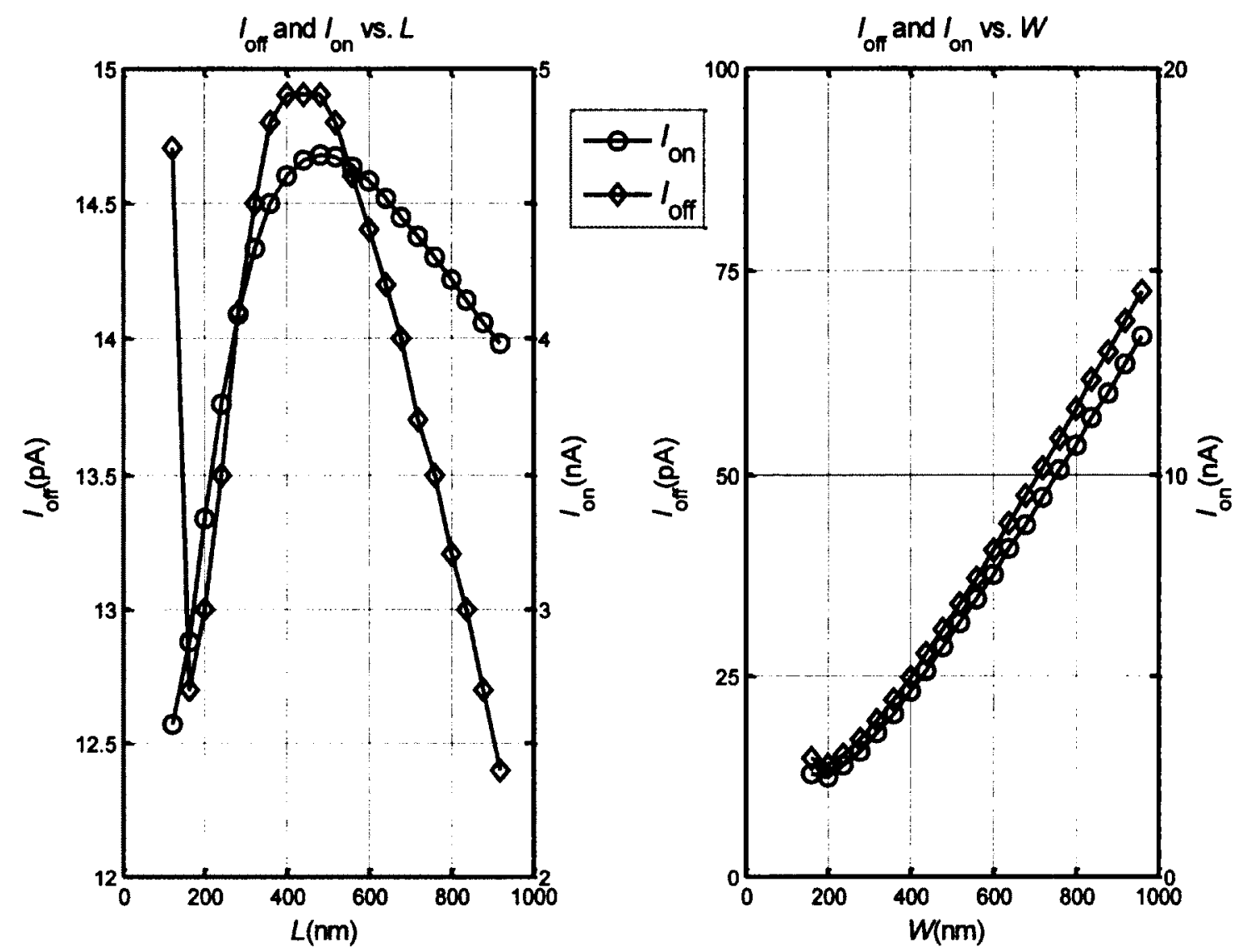

Figure $4.18 I_{\text {off }}$ and $I_{\text {on }}$ versus $L\left(@ W_{\min }\right)$ left figure, and versus $W\left(@ L_{\min }\right)$ right figure for PMOS transistor in IBM $130 \mathrm{~nm}$ technology at $V_{\mathrm{DD}}=0.2 \mathrm{~V}$.

Instead, we report an important figure of merit in CMOS digital circuits operating in the sub-threshold region, the $I_{\text {on }} / I_{\text {off }}$ ratio. The higher $I_{\text {on }} / I_{\text {off }}$ ratio means more robustness and immunity to noise. In designing sub-threshold SRAM, read and hold stability, and write ability, are strongly related to $I_{\text {on }} / I_{\text {off }}[9]$. Besides to robust subthreshold circuits design, $I_{\text {on }} / I_{\text {off }}$ ratio is an important factor in optimizing the leakage energy (Equation (3-26)) that has a large share in the total energy consumption in the sub-threshold region. $I_{\text {on }} / I_{\text {off }}$ is sketched in Figure 4.19 for each of four considered technologies. This figure shows that changing the channel length has more benefit in improving $I_{\text {on }} / I_{\text {off }}$ ratio than increasing the channel width, except for the PMOS transistor in the $180 \mathrm{~nm}$ technology. 

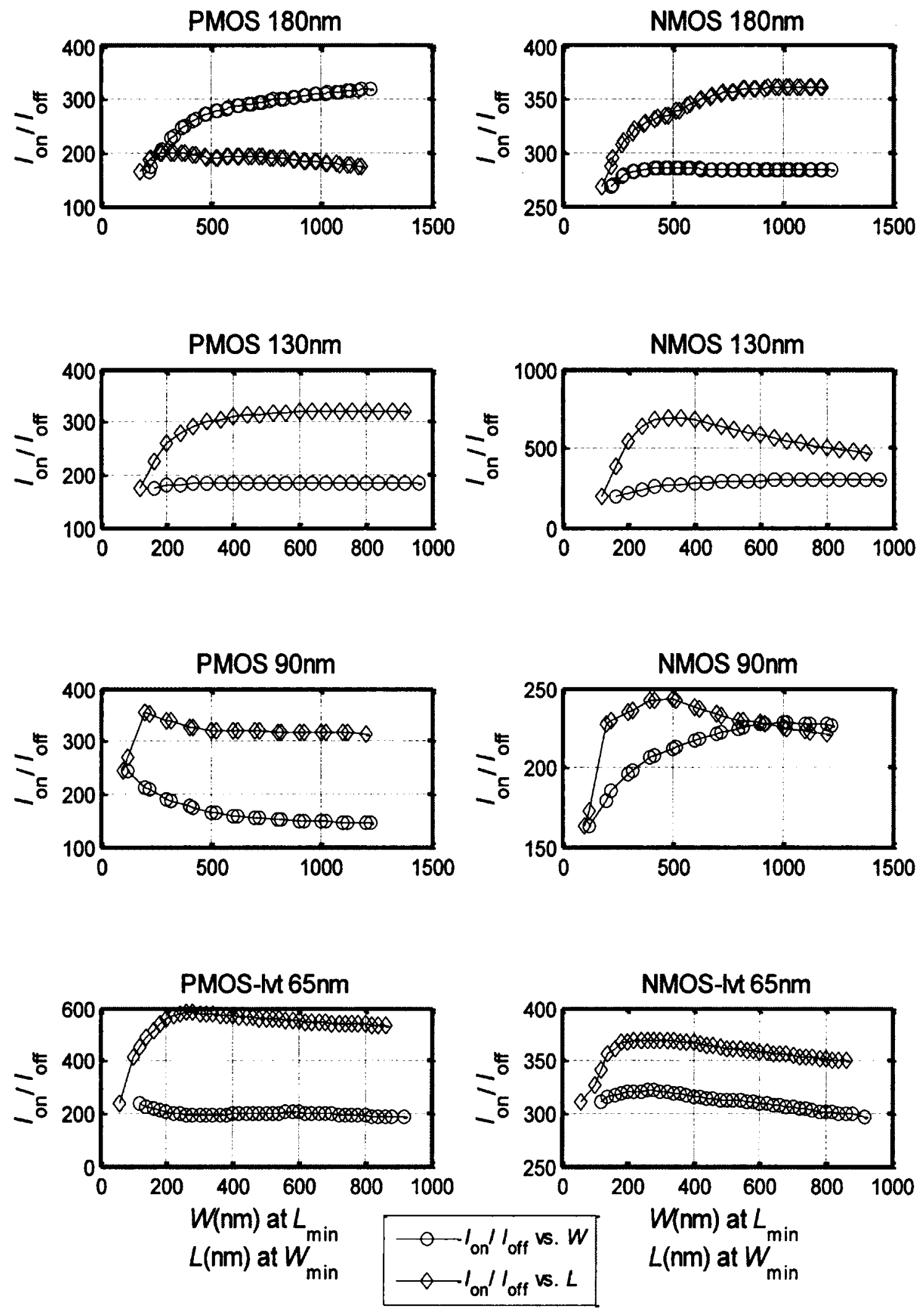

Figure $4.19 I_{\text {on }} / I_{\text {off }}$ versus $L\left(@ W_{\min }\right)$ and versus $W\left(@ L_{\min }\right)$ at $V_{\mathrm{DD}}=0.2$. 


\subsection{Sub-threshold Slope}

The sub-threshold slope, $S$, is an important factor in designing a CMOS digital circuit operating in the sub-threshold region. The parameter $S$ typically varies in the range of 70 to $100 \mathrm{mV} / \mathrm{dB}$ for the available CMOS technologies. A smaller $S$ for a transistor implies that the transistor can do faster transition between the "off" and "on" states, which means higher speed and less short-circuit energy consumption. Since $S$ is related to the depletion capacitance under the gate area, as expressed in Equation (3-7), it seems that transistor sizing will affect this parameter.

In this section we study the effects of the channel dimensions on this parameter. Figure 4.20 shows the variation of $S$ with respect to $W$ and $L$. As it shows, increasing $L$ to several folds of the minimum channel lengths, decreases $S$ which is more desirable for designing the sub-threshold digital circuits. However, increasing $W$ in some cases increases $S$ or slightly decreases it, except for the NMOS transistor in the $130 \mathrm{~nm}$ and the PMOS transistor in the $180 \mathrm{~nm}$.
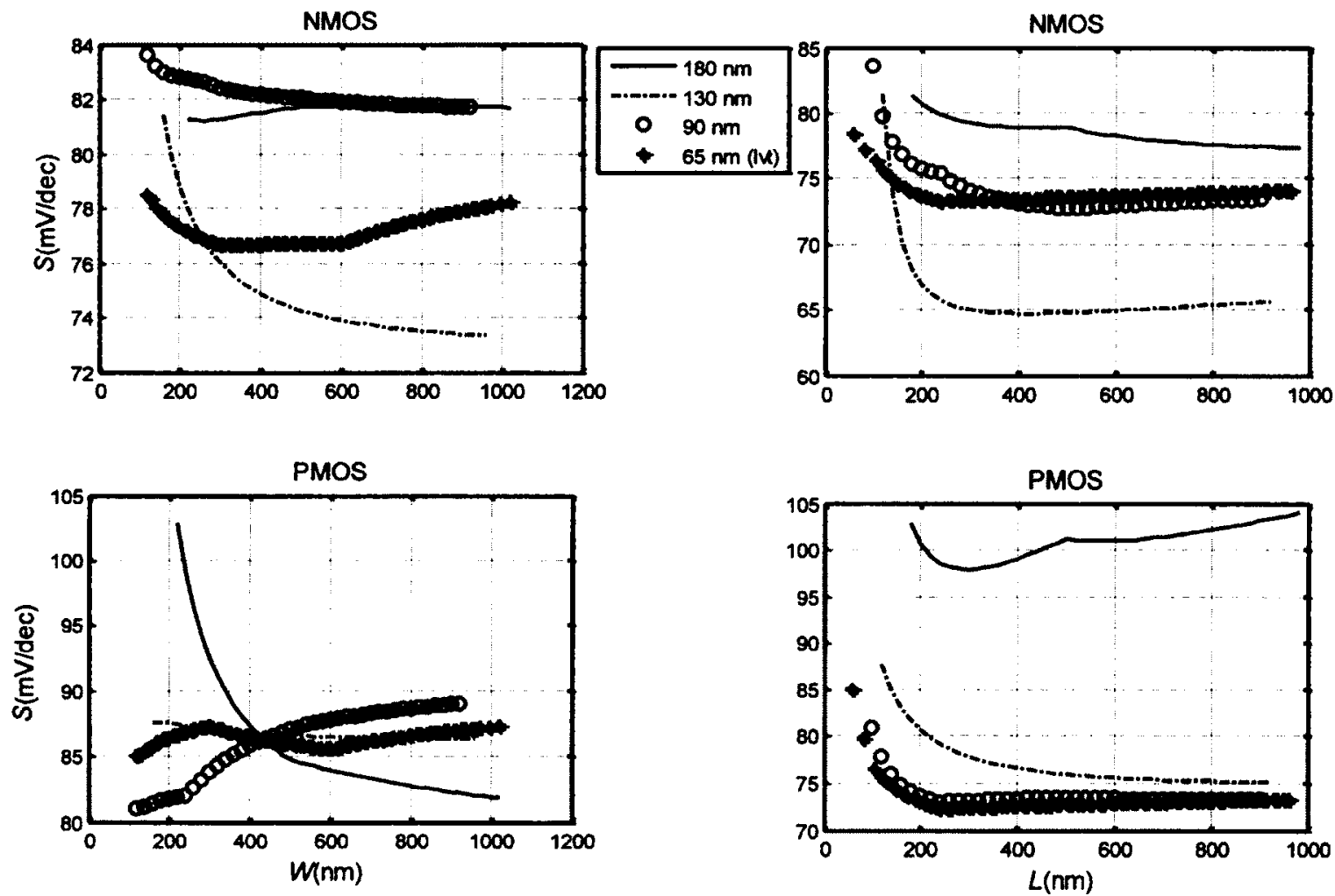

Figure 4.20 Sub-threshold slope versus W (@ $\left.L_{\min }\right)$ (left) and versus $L(@$ $\left.W_{\min }\right)($ right) for NMOS and PMOS transistors. 


\subsection{Chapter Summary}

In this chapter we studied the effects of transistor sizing on the threshold voltage of a MOSFET. Due to the exponential relationship between the sub-threshold current and the threshold voltage in the sub-threshold region, the current behavior in this mode of operation is not like the super-threshold current. In the super-threshold region the current has its maximum when the channel length is in its minimum value. This means that in the super-threshold operation the channel length is usually fixed to its minimum values, except in some special applications. ${ }^{2}$ However, the sub-threshold current shows a maximum point when the channel length is larger than minimum in most cases. This fact implies that in the sub-threshold operation for digital applications the channel length can be increased to maximize the deriving current.

Besides increasing the current driveability, increasing the channel length improves $I_{\text {on }}$ $/ I_{\text {off }}$ ratio. The higher $I_{\text {on }} / I_{\text {off }}$ ratio makes digital circuits operating in the sub-threshold region more reliable and more noise immune. Also, the leakage energy is related to $I_{\text {on }} / I_{\text {off }}$ ratio inversely; i,e., higher $I_{\text {on }} / I_{\text {off }}$ ratio means less leakage energy consumption.

Another benefit of increasing the channel length is obtaining a smaller sub-threshold slope. The smaller $S$ makes a faster transition from the "on" state to "off" state and vice versa, which means faster circuits and less short-circuit power consumption.

In the next chapter the effect of the channel length on the delay optimization is presented. A method to find the optimum channel length is proposed. A simple inverter RO is studied to verify the effectiveness of the proposed method. Then, the other transistor connections (serial and parallel) are studied to find the optimum channel for NAND and NOR gates.

\footnotetext{
${ }^{2}$ Current-mirrors with high output impedance in analog applications, and keepers in PTL logic gates in digital applications.
} 


\section{Delay Optimization in Sub-threshold Circuits}

Digital and analog circuits operating in the sub-threshold mode are widely used for ultra-low-power applications, e.g., biomedical devices. The current in the sub-threshold is typically 1000 times smaller than that of the super-threshold current. The main drawback of the sub-threshold circuits is their low speed (typically in the range 0 f $1-10 \mathrm{MHz}$ ) due to their small drive current. In some applications where the speed is not the main concern, this range of speed seems adequate. However, in some applications like mobile wireless devices, where both speed and energy consumption are important for designers, using the sub-threshold mode without improving the operation speed is impossible. There are a number of reports on speeding up sub-threshold circuits by manipulating the channel width and fingering wide transistors to narrower transistors [52] [53] [54] [55] [56] [73].

Here in this chapter we are proposing our own method based on the channel length manipulation. Increasing $L$ up to a few times of the minimum length (e.g., 2-3 times), causes a noticeable improvement in the performance of CMOS circuits operating in the sub-threshold region. Depending on the technology node, and the transistor type, and the supply voltage, the channel length where the performance becomes optimum changes. In the following sections we proposed a method for finding the optimum channel length and then verify it with some sample circuits. Throughout the work presented in this chapter, the channel width is fixed to its minimum value unless otherwise mentioned.

\subsection{Current-over-Capacitance (CoC)}

According to Equation (3-16), the propagation delay is proportional to $C / I$ ratio, where $C$ is the total capacitance connected to the node and $I$ is the driving current that charges or discharges $C$. In our proposed method we made an assumption that each transistor operates independently. In the high-to-low transitions we ignored the effects of the PMOS transistors and in the low-to-high transitions we ignored the effects of NMOS transistors. In other words, we optimize $t_{\mathrm{phh}}$ and $t_{\mathrm{phl}}$ individually and find the optimum 
channel length for each of NMOS and PMOS transistors individually. Although initially this method might seem inaccurate, it is a quick and still reliable solution. It spares the need for exhaustive blind simulations that are very time consuming, especially in large circuits with millions of transistors. Consider the case that an inverter is driving an identical one, as shown in Figure 5.1. The capacitances that are involved in the propagation delay are the drain junction capacitances of the first stage $\left(C_{\mathrm{DBN} 1}, C_{\mathrm{DBP} 1}\right)$ and the gate capacitances of the next stage $\left(C_{\mathrm{GGN} 2}, C_{\mathrm{GGP}}\right)$. As shown in this figure in the high-to-low transition $\mathrm{N} 1$ discharges the total capacitance $C$ and in the low-to-high transition P1 charges it. Therefore, we may rewrite Equation (3-16) as

$$
\begin{aligned}
t_{p l h} & =\frac{\left(C_{D B N 1}+C_{D B P 1}+C_{G G N 2}+C_{G G P 2}\right) V_{D D}}{2 I_{a v P 1}} \\
t_{p h l} & =\frac{\left(C_{D B N 1}+C_{D B P 1}+C_{G G N 2}+C_{G G P 2}\right) V_{D D}}{2 I_{a v N 1}}
\end{aligned}
$$

assuming $C_{\mathrm{DBN} 1}=C_{\mathrm{DBP1}}=C_{\mathrm{DB}}$ and $C_{\mathrm{GGN} 2}=C_{\mathrm{GGP} 2}=C_{\mathrm{GG}}$, that are valid approximation for all considered technologies, Equation (5-1) can be summarized as

$$
t_{p l h}=\frac{\left(C_{D B}+C_{G G}\right) V_{D D}}{I_{a v P 1}}, t_{p h l}=\frac{\left(C_{D B}+C_{G G}\right) V_{D D}}{I_{a v N 1}}
$$

To minimize the propagation delay, Equation (5-2) implies that the Current-overCapacitance $(\mathrm{CoC})$ ratio should be maximized. As explained in Section 4.2, the subthreshold current shows a maximum point as the channel length increases except for the NMOS transistor in the $180 \mathrm{~nm}$ technology and for the $65 \mathrm{~nm}$ technology for the svt flavour of transistors. Hence, it is acceptable to look for a channel length where $\mathrm{CoC}$ becomes maximum ( $L_{\mathrm{COC} \max }$ ). Also it is predictable that $L_{\mathrm{COC} \max }$ will be smaller than $L_{\mathrm{I} \max }$ introduced in Section 4.2. To find $L_{\text {CoCmax }}$ for each type of transistor in each of the four technologies, we biased an NMOS and a PMOS transistor with DC supplies; then measured the drain current and $C_{\mathrm{GG}}$ and $C_{\mathrm{DB}}$ to find the $\mathrm{CoC}$ ratio.

As discussed in Section 4.3, both $C_{\mathrm{GG}}$ and $C_{\mathrm{DB}}$ are voltage dependant. Since propagation delay is defined as the time from when the input signal passes $V_{\mathrm{DD}} / 2$ until the 
output signal passes $V_{\mathrm{DD}} / 2$, we calculate an average value for the current, $C_{\mathrm{GG}}$, and $C_{\mathrm{DB}}$ in these two cases to calculate $\mathrm{CoC}$ :

$$
1-V_{\mathrm{gs}}=V_{\mathrm{DD}} / 2, V_{\mathrm{ds}}=V_{\mathrm{DD}} \quad 2-V_{\mathrm{gs}}=V_{\mathrm{DD}}, V_{\mathrm{ds}}=V_{\mathrm{DD}} / 2
$$
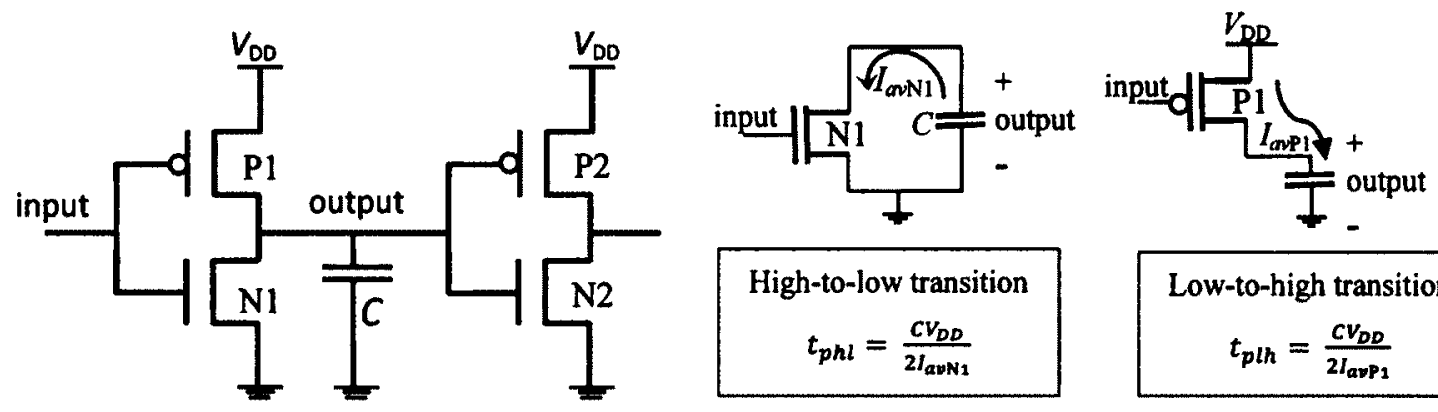
High-to-low transition $t_{p h l}=\frac{C V_{D D}}{2 l_{a v N_{1}}}$ Low-to-high transition

$$
t_{p l h}=\frac{C v_{D D}}{2 I_{\text {avp } 1}}
$$

$$
C=C_{\mathrm{DBP} 1}+C_{\mathrm{DBN} 1}+C_{\mathrm{GGP} 2}+C_{\mathrm{GGN} 2}
$$

Figure 5.1 An inverter driving an identical inverter. High-to-low and low-tohigh transitions are illustrated.

Next, the $\mathrm{CoC}$ ratio versus the channel length is plotted for both types transistor. As a sample, Figure 5.2 shows the maximum point for $\mathrm{CoC}$ versus $L$ for PMOS and NMOS transistors in the IBM $130 \mathrm{~nm}$ technology at $V_{\mathrm{DD}}=0.2 \mathrm{~V}$. It is important to notice that $\mathrm{CoC}$ curves are nearly flat in the vicinity of their maximum points. This means that using

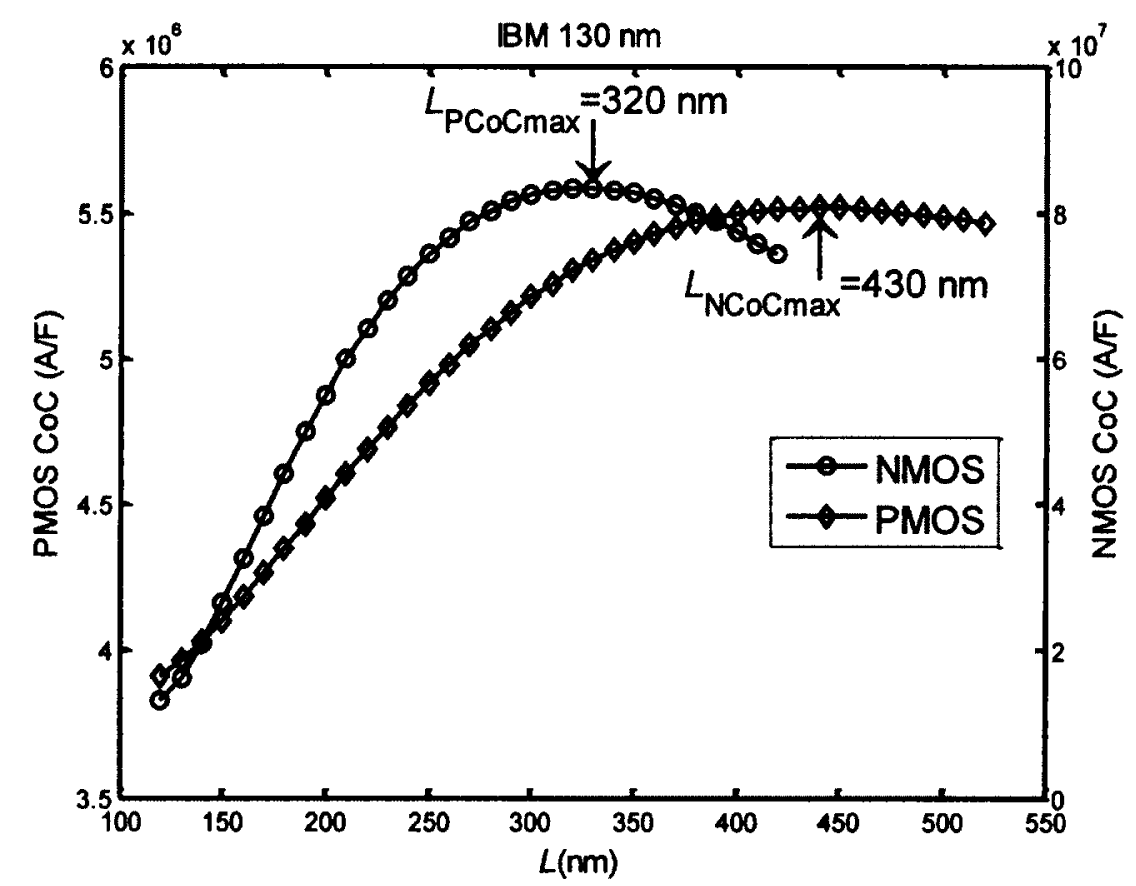

Figure 5.2 CoC versus $L$ for NMOS and PMOS transistors in IBM $130 \mathrm{~nm}$ technology at $V_{\mathrm{DD}}=0.2 \mathrm{~V}$ 
channel lengths slightly smaller than the maximum points would not affect the speed significantly, but make the power consumption to be less.

The other important point that we should notice is the dependence of $L_{\mathrm{CoCmax}}$ to the supply voltage. As the supply voltage increases towards the super-threshold region, the optimum channel length decreases towards the minimum length in the technology. Figure 5.3 shows $\mathrm{CoC}$ versus the channel length for the PMOS-lvt transistor in the TSMC $65 \mathrm{~nm}$ at two different supply voltages.

Moreover, for the transistors where the sub-threshold current is a descending function of the channel length, e.g., the NMOS transistor in the TSMC $180 \mathrm{~nm}, \mathrm{CoC}$ is also a descending function of the channel length and the optimum channel length (maximizing $\mathrm{CoC}$ ) is the minimum channel length of the technology, as shown in Figure 5.4. However, this does not mean that for a transistor whit a maximum point in its subthreshold current curve, there would be definitely a maximum point in the $\mathrm{CoC}$ curve. For instance, although the sub-threshold current for the NMOS transistor in the TSMC

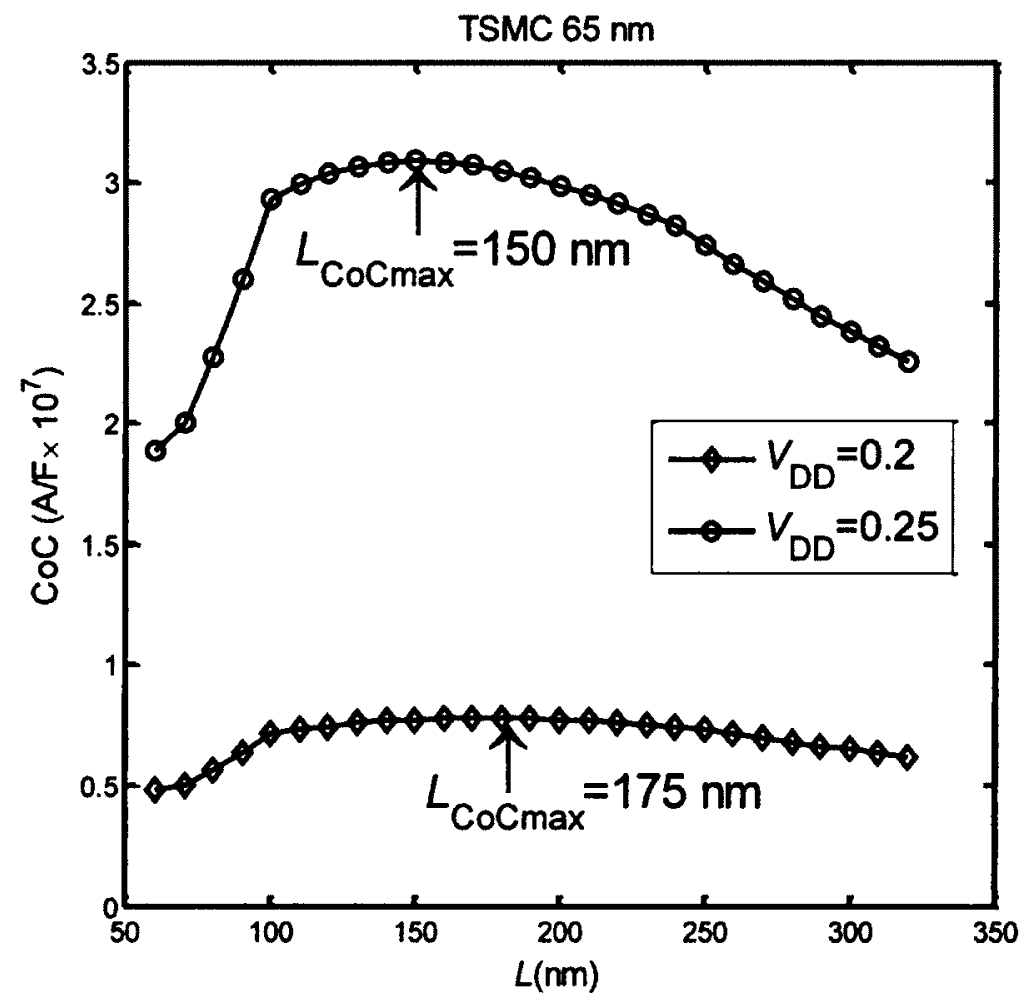

Figure 5.3 CoC versus $L$ for PMOS-lvt in TSMC $65 \mathrm{~nm} \mathrm{LP}$ at two different supply voltages. 
$90 \mathrm{~nm}$ technology shows a maximum point with respect to the channel length, the CoC versus the channel length curve for this transistor shows no optimum point. That is, the maximum $\mathrm{CoC}$ occurs at the minimum channel length as shown in Figure 5.4. Thus unlike to what is done in [8], only considering the current versus channel length curve is not sufficient for delay optimization.
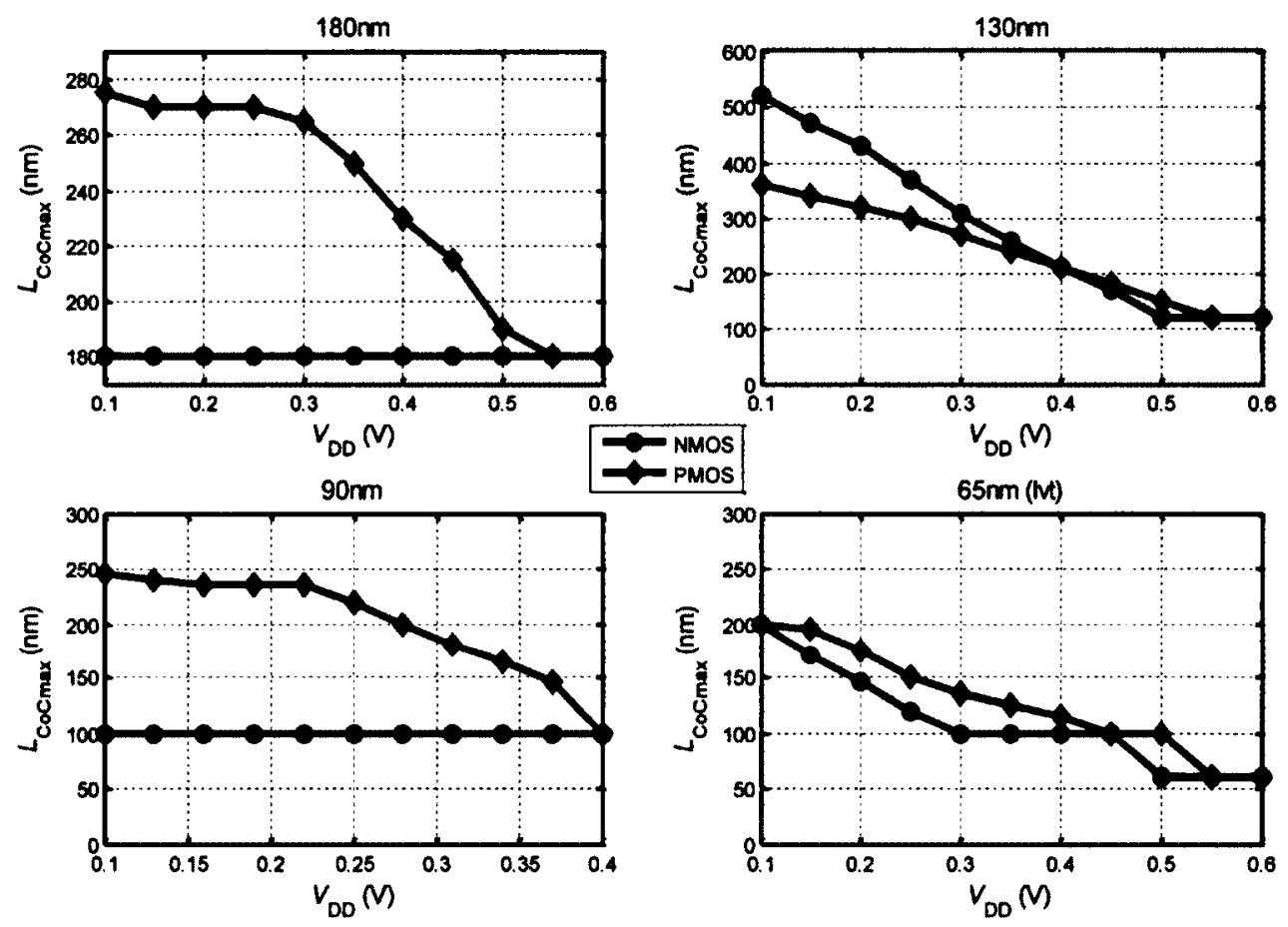

Figure 5.4 $L_{\text {CoCmax }}$ versus $V_{\text {DD }}$ -

\subsection{Delay versus Channel Length}

To verify the effectiveness of the $\mathrm{CoC}$ method proposed in the previous section, in this section we will plot the delay for a circuit versus the channel length of its transistors. Our test circuit is an inverter like that of Figure 5.1. We applied a square wave with equal rise and fall times to the input and measured the propagation delay from the input node to the output node. Then, we plotted a 3D graph of the delay versus both the NMOS and PMOS transistors channel lengths to find the channel lengths where the delay in minimum, $L_{\mathrm{Dmin}}$ (Figure 5.5). The same procedure was followed for all four considered technologies and the results are relatively close to what was obtained from the $\mathrm{CoC}$ simulation. The 3D plot shows that for a wide range of variations in the channel lengths 
of the NMOS and PMOS transistors, the delay varies between 25 to $28 \mathrm{~ns}$, which is a variation of about $10 \%$. Contour plots presented in the same figure shows the $L_{\mathrm{Dmin}}$ $\left(L_{\mathrm{p}}=350 \mathrm{~nm}\right.$ and $L_{\mathrm{n}}=420 \mathrm{~nm}$ ). The minimum delay shows a $35 \%$ improvement compared to the delay of the minimum-size circuit. The contour plot shows a flatness of the delay around its minimum point. The delay is almost constant inside the dotted oval in the contour plot for the range of $270 \mathrm{~nm}<L_{\mathrm{p}}<420 \mathrm{~nm}$ and $350 \mathrm{~nm}<L_{\mathrm{n}}<500 \mathrm{~nm}$. In Figure 5.2 , the CoC curve shows two different values for the optimum $L_{\mathrm{p}}$ and $L_{\mathrm{n}}$ than the values
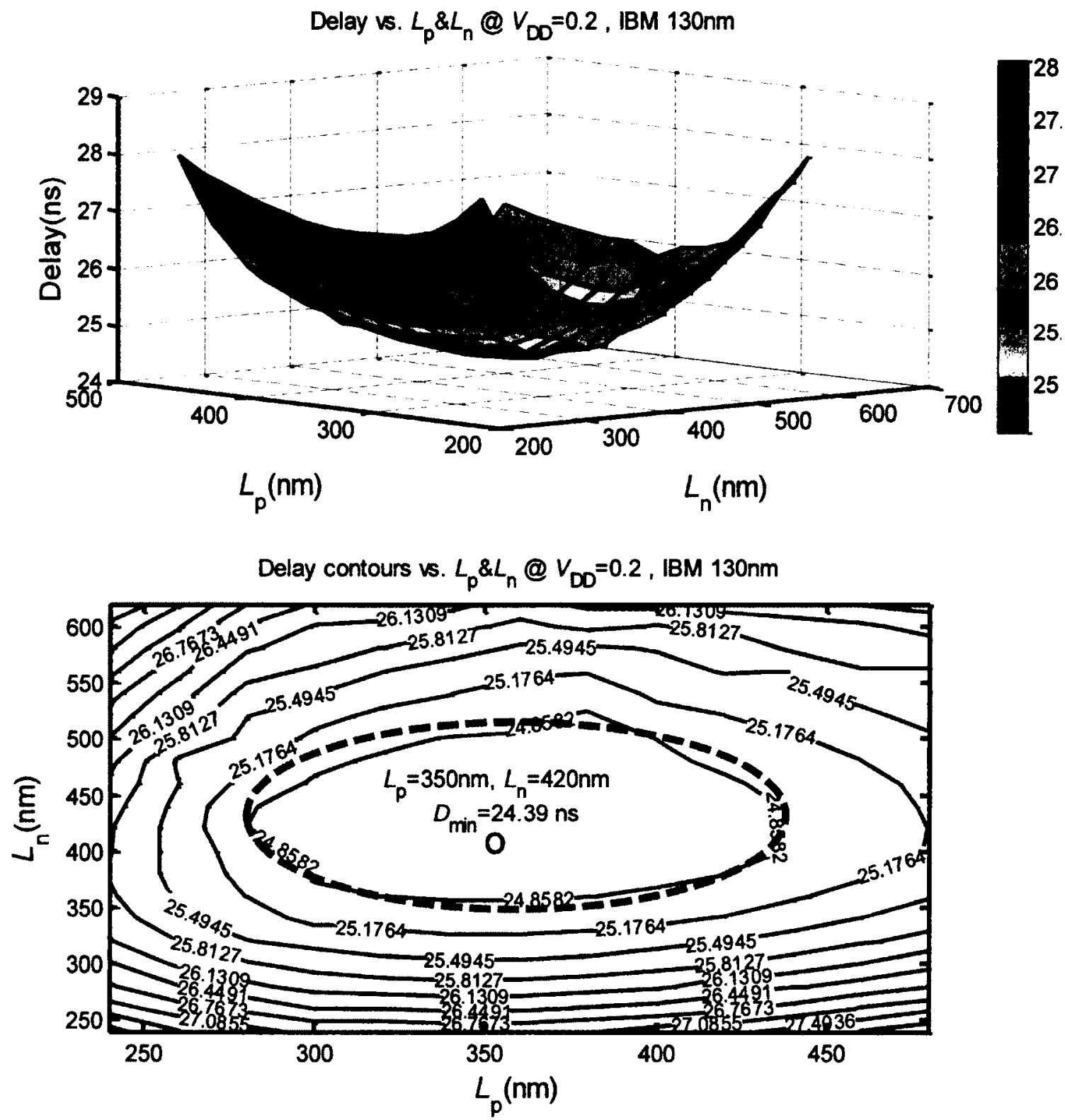

Figure 5.5 Delay versus $L_{\mathrm{p}}$ and $L_{\mathrm{n}}$ for an inverter driving an identical inverter. 3D plot (top) and contour plot (bottom) simulated in IBM $130 \mathrm{~nm}$ at $V_{\mathrm{DD}}=0.2 \mathrm{~V}$. 
obtained from the delay contour plot. However, they are still close enough to produce the minimum delay and can be chosen as the starting point for our simulations. Moreover, the channel lengths obtained from the $\mathrm{CoC}$ simulation are located inside the dotted oval in the contour plot. This implies that if we use the $L_{\mathrm{CoCmax}}$ in an inverter, then its delay is almost equal to the absolute minimum delay. By applying the same procedure to the other technologies, the same results are obtained. As a further example, Figure 5.6 illustrates the contour plot of the delay for an inverter consisting of lvt-type transistors in the $65 \mathrm{~nm}$ technology. This plot indicates that if the channel lengths change $L_{\mathrm{Dmin}}$ to any values inside the dotted oval, the delay only increases by $0.7 \%$.

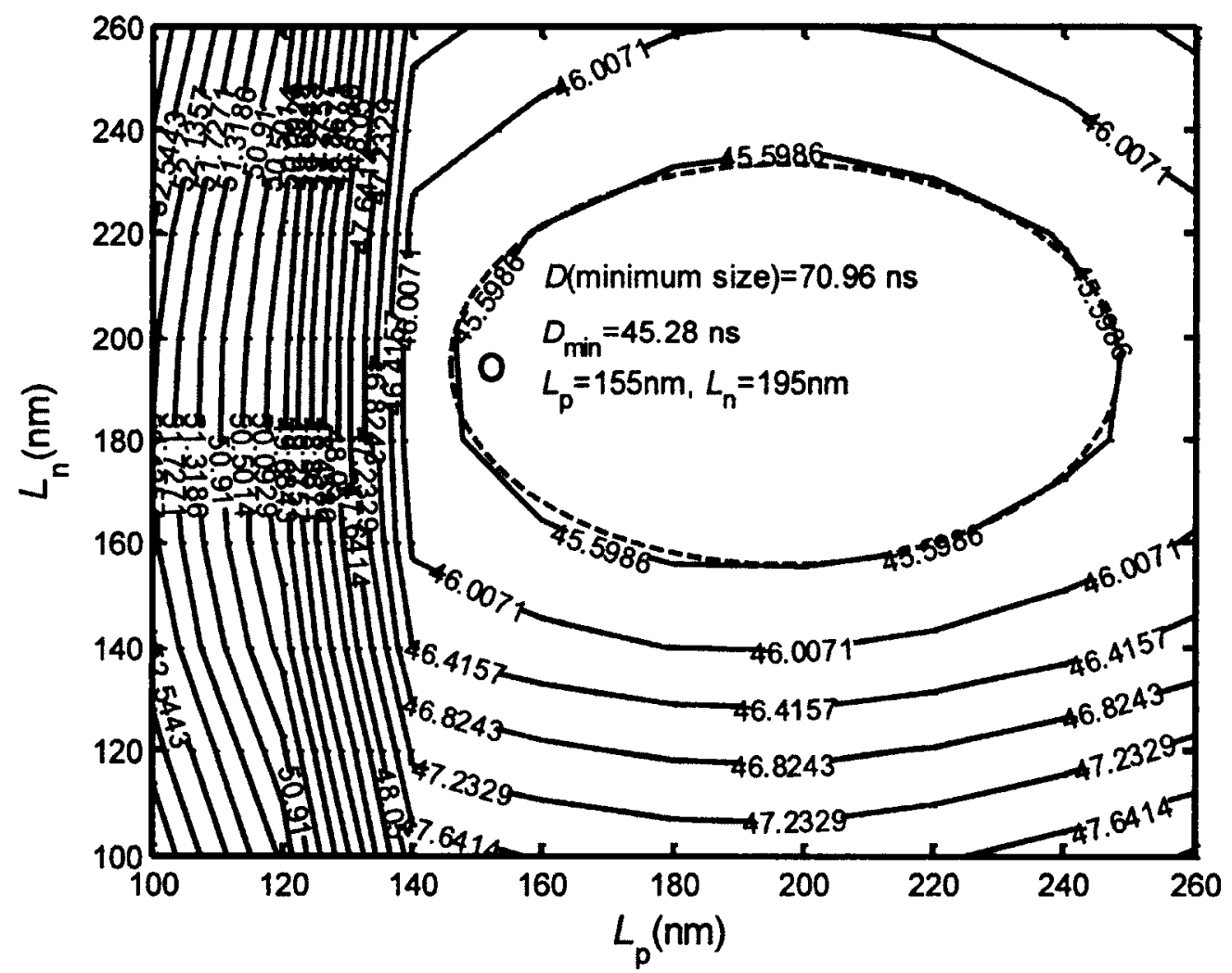

Figure 5.6 Delay contours versus $L_{\mathrm{p}}$ and $L_{\mathrm{n}}$ in TSMC $65 \mathrm{~nm}$ at $V_{\mathrm{DD}}=0.2 \mathrm{~V}$.

In Figure 5.7, we plotted the delay versus the power supply for three different sets of transistor sizing in three technologies. One of the curves shows the delay for minimumsize transistors. The two other curves show the delay for the channel lengths obtained from the $\mathrm{CoC}$ and delay optimization. These two curves are almost identical and no difference can be noticed between the two. 

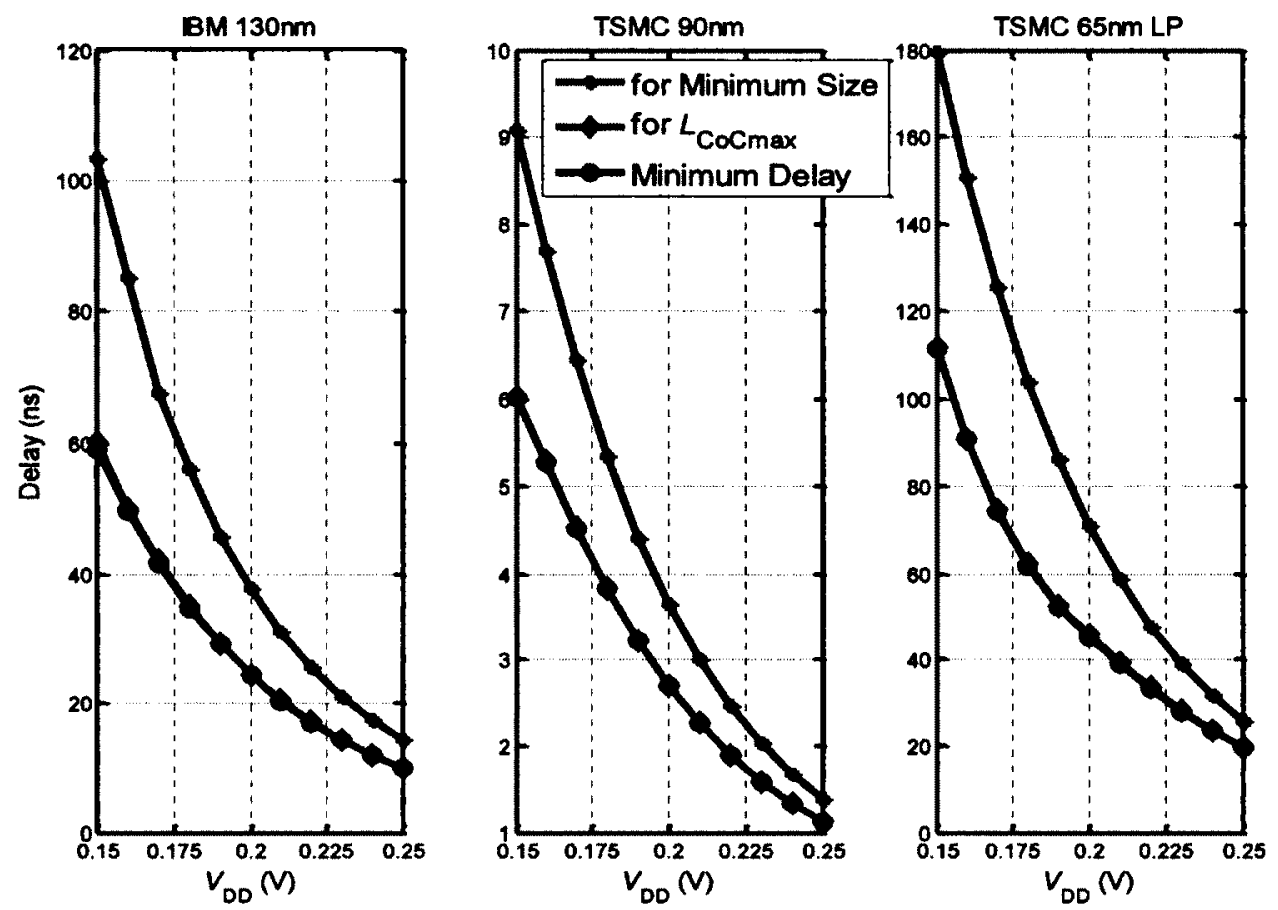

Figure 5.7 Delay versus supply voltage measured for three different sets of transistors sizing.

In Table 5.1the result of delay results (based on simulation) for all four technologies are presented at $V_{\mathrm{DD}}=0.2 \mathrm{~V}$. Despite the discrepancy between $L_{D \min }$ and $L_{\mathrm{CoCmax}}$, the delay for these two sets of channel lengths shows complete compliance. In the $\mathrm{CoC}$ method each transistor is studied individually, which does not replicate the real case for an inverter. In an inverter both transistors are involved in the delay and we cannot neglect the influence of the transistor that is going to the "off" state, especially in the subthreshold region where the off-current of the NMOS transistor and the on-current of the PMOS transistor are comparable.

Table 5.1 Delay measured for three different sets of transistor sizing at $V_{\mathrm{DD}}=0.2 \mathrm{~V}$.

\begin{tabular}{|c|c|c|c|c|c|c|c|c|c|c|c|}
\hline & \multicolumn{3}{|c|}{ Minimum size } & \multicolumn{4}{|c|}{$L_{D_{\min }}$} & \multicolumn{4}{|c|}{$L_{\text {CoCmax }}$} \\
\hline & 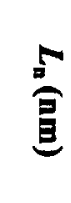 & $\stackrel{\text { D }}{\text { 总 }}$ & 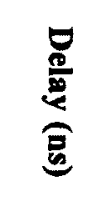 & 䍖 & $\stackrel{\text { 畐 }}{\text { 足 }}$ & 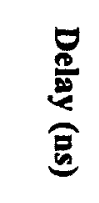 & $\begin{array}{r}\bar{g} \\
0 \\
0 \\
0 \\
0\end{array}$ & $\stackrel{N}{\stackrel{D}{E}}$ & $\stackrel{\text { 兽 }}{0}$ & 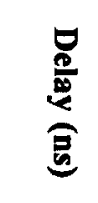 & 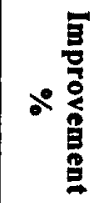 \\
\hline $180 \mathrm{~nm}$ & 180 & $\overline{180}$ & 2373 & 180 & 270 & 1880 & 20.7 & 180 & 270 & 1880 & 20.7 \\
\hline $130 \mathrm{~nm}$ & 120 & 120 & 37.84 & 420 & 350 & 24.39 & 35.5 & 430 & 320 & 24.40 & 35.5 \\
\hline $90 \mathrm{~nm}$ & 100 & 100 & 3.647 & 100 & 240 & 2.71 & 25.7 & 100 & 240 & 2.71 & 25.7 \\
\hline $65 \mathrm{~nm}$ & 60 & 60 & 70.96 & 195 & 155 & 45.28 & 36.2 & 145 & 170 & 45.84 & 35.4 \\
\hline
\end{tabular}




\subsection{Maximizing the Frequency of a RO}

When comparing the performance of digital gates implemented in different technologies, it is important to have a uniform way of measuring the propagation delay so that technologies can be judged on an equal base. The standard circuit for delay measurement is the RO, consisting of an odd number of inverters connected in a circular chain configuration such that the output of the last inverter is fed back to the input of the first one [81]. The frequency of oscillation of a RO is determined by

$$
f=\frac{1}{2 N t_{p}}
$$

where $N$ is the number of inverters in the loop and $t_{p}$ is the propagation delay of a single inverter in the loop. The lower delay of the inverter results in the higher frequency of the RO.

To find the channel lengths resulting in maximum frequency $\left(L_{\mathrm{fmax}}\right)$, we plotted a 3D plot of the frequency versus $L_{\mathrm{p}}$ and $L_{\mathrm{n}}$. Then, $L_{\mathrm{fmax}}$ is extracted from the plot. For example, Table 5.2 presents the simulation results for ROs with 9 and 29 inverters in two sets of transistor sizes. This table shows that $L_{\mathrm{fmax}}$ is independent of the number of inverters in the RO and only depends on the technology node.

$L_{\text {fmax }}$ differs from both $L_{\text {CoCmax }}$ and $L_{\mathrm{Dmin}}$ introduced in Sections 5.1 and 5.2 in $130 \mathrm{~nm}$ and $65 \mathrm{~nm}$ technologies. This discrepancy originates from the dependence of the propagation delay to the slope of the input. In the $\mathrm{CoC}$ study presented in Section 5.1, we studied the DC behaviour of a transistor at two bias points defined to measure the delay. When we studied the delay minimization in Section 5.2, we applied an input with equal rise and fall times, which is not the real case in practice. In a RO, where an inverter is driven by another inverter, the rise and fall times are usually different. Moreover, rising and falling times depend on the driving stage, whereas in Section 5.2 the driving pulse is fed from an external source whit fixed rising and falling times. All these simplifications used in finding $L_{\mathrm{CoCmax}}$ and $L_{\mathrm{Dmin}}$ result in reported differences. 
Table 5.2 Frequency for a RO with 9 and 29 inverters simulated for two different sets of transistor sizing at $V_{\mathrm{DD}}=0.2 \mathrm{~V}$.

\begin{tabular}{|c|c|c|c|c|c|c|c|c|}
\hline & \multicolumn{3}{|c|}{ Minimum size } & \multicolumn{4}{|c|}{$L_{\text {fmax }}$} \\
\hline & & 兽 & $\stackrel{5}{\stackrel{5}{\mathrm{~B}}}$ & 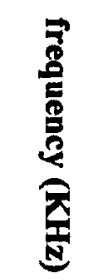 & $\stackrel{-}{\mathrm{B}}$ & $\underset{\mathrm{D}}{\stackrel{0}{\mathrm{E}}}$ & 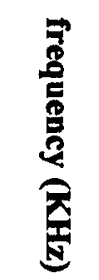 & $\begin{array}{l}5 \\
\mathbf{3} \\
0 \\
0 \\
0 \\
0 \\
0 \\
0 \\
0\end{array}$ \\
\hline \multirow{2}{*}{$180 \mathrm{~nm}$} & $9 \mathrm{INV}$ & 180 & 180 & 28.4 & 180 & 270 & 34 & 19.7 \\
\hline & $29 \mathrm{INV}$ & 180 & 180 & 8.83 & 180 & 270 & 10.6 & 20 \\
\hline \multirow{2}{*}{$130 \mathrm{~nm}$} & $9 \mathrm{INV}$ & 120 & 120 & 1440 & 340 & 380 & 2810 & 95.1 \\
\hline & $29 \mathrm{INV}$ & 120 & 120 & 446 & 340 & 380 & 873 & 95.7 \\
\hline \multirow{2}{*}{$90 \mathrm{~nm}$} & $9 \mathrm{INV}$ & 100 & 100 & 14390 & 100 & 240 & 23830 & 65.6 \\
\hline & $29 \mathrm{INV}$ & 100 & 100 & 4466 & 100 & 240 & 7400 & $\overline{65.7}$ \\
\hline \multirow{2}{*}{$65 \mathrm{~nm}$} & $9 \mathrm{INV}$ & 60 & 60 & 1584 & 150 & 240 & 3004 & 89.7 \\
\hline & $29 \mathrm{INV}$ & 60 & 60 & 491 & 150 & 240 & 932 & 89.8 \\
\hline
\end{tabular}

Although $L_{\mathrm{fmax}}$ differs from $L_{\mathrm{CoCmax}}$ and $L_{\mathrm{Dmin}}$ especially in 130 and $65 \mathrm{~nm}$ technologies, the frequency reported for these three sets of channel lengths does not show a big difference. Table 5.3 lists the results for a 29 inverter $\mathrm{RO}$ at $V_{\mathrm{DD}}=0.2 \mathrm{~V}$. Parameter $\Delta \mathrm{f}$ in this table defines the difference between the frequency resulted by incorporating $L_{\mathrm{Dmin}}$ and $L_{\mathrm{COC} \max }$ in the RO and the maximum frequency obtained by $L_{\text {fmax }}$. The table reveals that the three results are comparable.

Note that according to Figure $5.4, L_{\mathrm{CoCmax}}$ is a function of the supply voltage. Hence, we studied the dependence of the maximum frequency and $L_{\mathrm{fmax}}$ of a 29 inverter RO to the supply voltage. Since for the $180 \mathrm{~nm}$ and $90 \mathrm{~nm}$ technologies $L_{\mathrm{fmax}}$ exactly matches $L_{\text {CoCmax }}$, we have not reported any results of the simulations for these two technologies. The results of the simulations for the $130 \mathrm{~nm}$ and $65 \mathrm{~nm}$ technologies show an acceptable margin between the maximum frequency and the frequency achieved by using $L_{\text {CoCmax }}$ for the supply voltages variation from $150 \mathrm{mV}$ to $250 \mathrm{mV}$. As an example, Table 5.4 shows the results of the simulation done for the $65 \mathrm{~nm}$ technology. Incorporating $L_{\mathrm{CoCmax}}$ 
degrades the frequency of the RO by a maximum $3.3 \%$, but with a smaller area which means a lower cost and energy consumption.

Table 5.3 Frequency in a 29 inverter $\mathrm{RO}$ simulated for three sets of channel lengths at $V_{\mathrm{DD}}=0.2 \mathrm{~V}$.

\begin{tabular}{|c|c|c|c|c|c|c|c|c|c|c|c|}
\hline & \multicolumn{3}{|c|}{$L_{\mathrm{fmax}}$} & \multicolumn{4}{|c|}{$L_{D_{\min }}$} & \multicolumn{4}{|c|}{$L_{C O C \max }$} \\
\hline & 兽 & $\stackrel{\text { 总 }}{\text { 兽 }}$ & 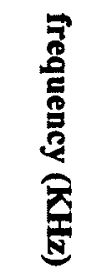 & 嚍 & $\stackrel{5}{\text { 总 }}$ & 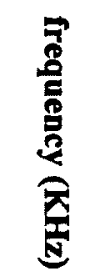 & $\underset{0^{\circ}}{R}$ & $\stackrel{\text { 点 }}{\mathrm{B}}$ & $\stackrel{\text { 音 }}{\mathrm{B}}$ & 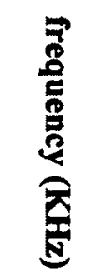 & $\underset{d^{\circ}}{R}$ \\
\hline $180 \mathrm{~nm}$ & 180 & 270 & 10.6 & 180 & 270 & 10.6 & 0 & 180 & 270 & 10.6 & 0 \\
\hline $130 \mathrm{~nm}$ & 340 & 380 & 873 & 420 & 350 & 869 & -0.46 & 430 & 320 & 867 & -0.69 \\
\hline $90 \mathrm{~nm}$ & 100 & 240 & 7400 & 100 & 240 & 7400 & 0 & 100 & 240 & 7400 & 0 \\
\hline $65 \mathrm{~nm}$ & 150 & 240 & 932 & 195 & 155 & 887 & -4.8 & 145 & 170 & 908 & -2.5 \\
\hline
\end{tabular}

Table 5.4 Frequency for a 29 inverter RO in different supply voltages for three different sets of channel lengths for TSMC $65 \mathrm{~nm}$ LP.

\begin{tabular}{|c|c|c|c|c|c|c|c|c|c|c|c|}
\hline \multirow[b]{2}{*}{$V_{\mathrm{DD}}$} & \multicolumn{3}{|c|}{$L_{\text {fmax }}$} & \multicolumn{4}{|c|}{$L_{D_{\min }}$} & \multicolumn{4}{|c|}{$L_{\text {COCmax }}$} \\
\hline & 䓃 & $\stackrel{5}{\overparen{B}}$ & 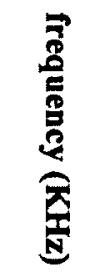 & $\stackrel{\tilde{g}}{\mathrm{~g}}$ & 兽 & 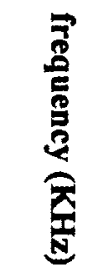 & $\underset{2}{\stackrel{R}{0}}$ & $\stackrel{\text { 嘼 }}{\mathrm{E}}$ & 息 & 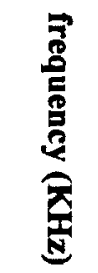 & $\underset{0}{\stackrel{2}{2}}$ \\
\hline 0.15 & 170 & 240 & 285 & 160 & 230 & 284.6 & -0.1 & 170 & 195 & 281 & -1.4 \\
\hline 0.16 & 170 & 240 & 362 & 180 & 215 & 360 & -0.6 & 165 & 190 & 356 & -1.7 \\
\hline 0.17 & 170 & 240 & 459 & 185 & 200 & 454 & -1.1 & 155 & 185 & 450.5 & -1.9 \\
\hline 0.18 & 160 & 240 & 583 & 195 & 185 & 569 & -2.4 & 155 & 180 & 570 & -2.2 \\
\hline 0.19 & 160 & 240 & 737 & 195 & 165 & 709 & -3.8 & 150 & 175 & 720 & -2.3 \\
\hline 0.2 & 150 & 240 & 932 & 195 & 155 & 887 & -4.8 & 145 & 170 & 908 & -2.5 \\
\hline 0.21 & 150 & 240 & 1176 & 185 & 150 & 1170 & -0.5 & 140 & 165 & 1143 & -2.8 \\
\hline 0.22 & 140 & 230 & 1480 & 175 & 150 & 1412 & -4.5 & 135 & 160 & 1436 & -3 \\
\hline 0.23 & 140 & 220 & 1857 & 165 & 145 & 1787 & -24 & 130 & 155 & 1799 & -3.1 \\
\hline 0.24 & 130 & 220 & 2324 & 155 & 140 & 2211 & -4.9 & 125 & 150 & 2247 & -3.3 \\
\hline 0.25 & 130 & 210 & 2899 & 150 & 135 & 2748 & $\begin{array}{l}-5.2 \\
\end{array}$ & 120 & 150 & 2813 & -3 \\
\hline
\end{tabular}


Table 5.1,Table 5.3, and Table 5.4 show that using $L_{\text {CoCmax }}$ results in frequencies that are comparable to the maximum obtainable frequency at different supply voltages. However, as introduced in Chapter 1, there are some other quality metrics for a digital circuit. These quality metrics could be in the same level of importance as the delay (frequency). The energy consumption and reliability of a digital circuit are two important factors in designing a digital circuit. The reliability is of special interest in the subthreshold region, where the supply voltage is very small and noise can cause problems for circuit functionality.

One measure of a digital gate's reliability is its VTC. Figure 5.8 shows the VTC of an inverter with minimum size transistors and three other different sets of channel lengths introduced in previous sections. For $L_{\mathrm{fmax}}, L_{\mathrm{Dmin}}$, and $L_{\mathrm{COC} \max }$, VTC plots are almost identical and there is no significant difference between them. The noise margins obtained from these four curves are presented in Table 5.5. Using $L_{\mathrm{fmax}}$ results in the largest SNM in comparison to the other three cases. However, $L_{\mathrm{COC} \max }$ and $L_{\mathrm{Dmin}}$ slightly deteriorate the SNM for marginal savings in energy, as confirmed by Table 5.5. In this table, the energy consumption per cycle for a RO with 29 minimum size inverters are compared to that of ROs with inverters using $L_{\mathrm{fmax}}, L_{\mathrm{Dmin}}$, and $L_{\mathrm{COC} \max }$ sets of channel lengths. The results

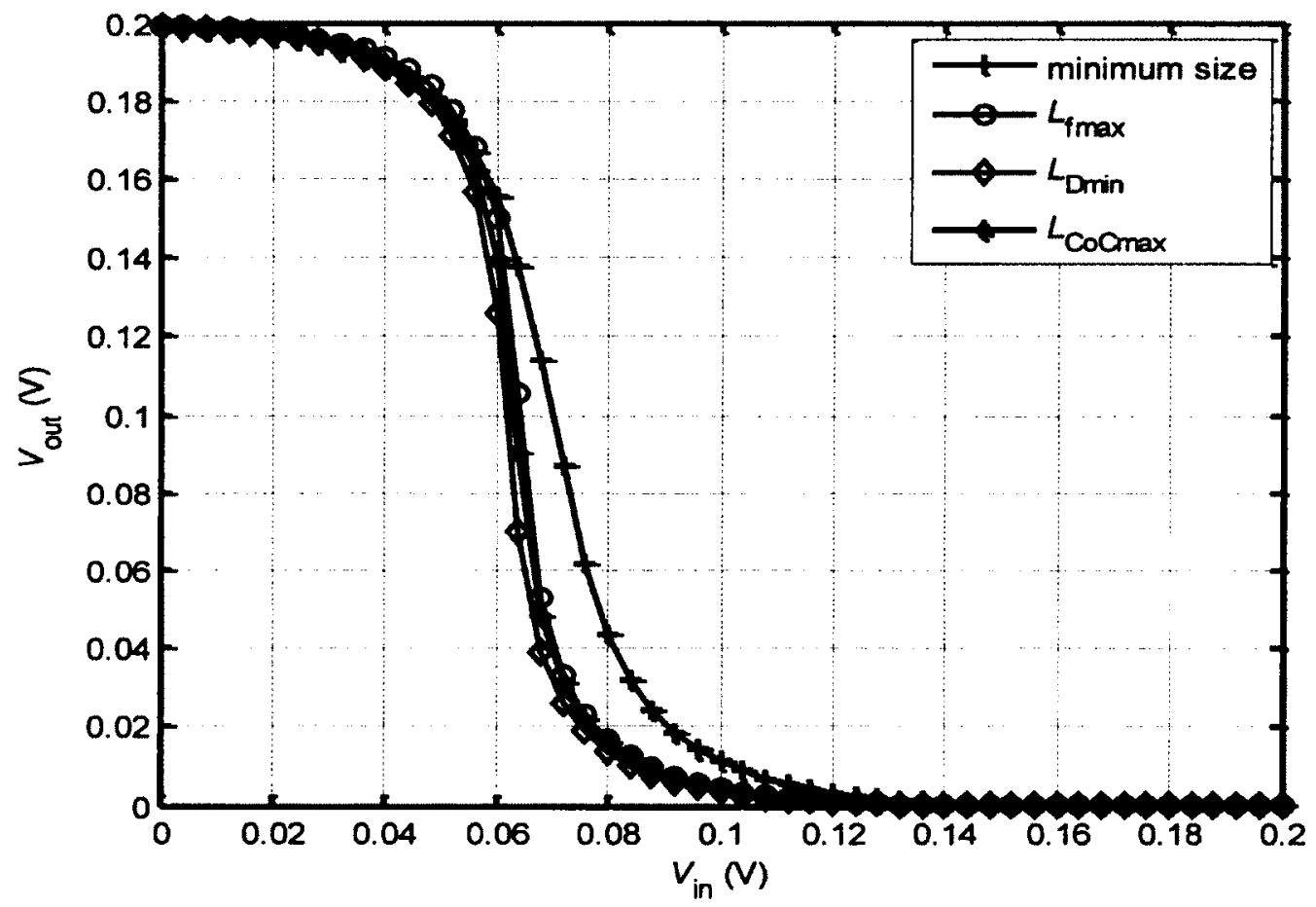

Figure 5.8 VTC for an inverter plotted for four sets of channel lengths in $65 \mathrm{~nm}$ at $V_{\mathrm{DD}}=0.2 \mathrm{~V}$. Both NMOS and PMOS transistors are "Jvt" types. 
show that among these three sets of channel lengths, the RO with $L_{\text {CoCmax }}$ has smaller energy consumption.

In order to summarize Sections 5.1-5.3, we may say that:

- In the $180 \mathrm{~nm}$ and $90 \mathrm{~nm}$ technologies, the channel length of the NMOS transistor should be kept at its minimum to maximize $\mathrm{CoC}$, but the channel length for the PMOS transistor must be increased to maximize its $\mathrm{CoC}$. The channel lengths obtained for maximizing the frequency of a RO $\left(L_{\mathrm{fmax}}\right)$ and the channel lengths obtained for minimizing the delay for an inverter $\left(L_{\mathrm{Dmin}}\right)$ and maximizing $\mathrm{CoC}\left(L_{\mathrm{CoCmax}}\right)$ closely match.

- In the $130 \mathrm{~nm}$ and $65 \mathrm{~nm}$ technologies, both the NMOS and PMOS transistors should have a channel length longer than the minimum to maximize $\mathrm{CoC}$. Simulations for minimizing the delay or maximizing the frequency show that the optimum channel lengths for the delay or frequency are larger than the minimum channel length. In these technologies, $L_{\mathrm{fmax}}, L_{\mathrm{Dmin}}$, and $L_{\mathrm{CoC} \max }$ sets of the channel lengths differ from each other. Incorporating $L_{\mathrm{COC} \max }$ in an inverter shows reasonable quality metrics such as delay, frequency, SNM, and energy consumption.

Based on the previous discussion, the upcoming question should be answered. Is $L_{\text {CoCmax }}$ appropriate for logic gates more complex than the inverter, e.g., NAND or NOR gates? If we use $L_{\text {CoCmax }}$ in a $\mathrm{RO}$ constructed with more complex gates, do we still get reasonable results? In the following section this question is answered.

Table 5.5 Noise margins for an inverter in three sets of channel length compared to that of the minimum size inverter. Energy per cycle and frequency operation of a 29 inverter RO compared for these four sets of channel lengths.

\begin{tabular}{|c|c|c|c|c|}
\hline $\begin{array}{c}\text { Quality } \\
\text { Metric }\end{array}$ & $L_{\text {minsize }}$ & $L_{\text {fmax }}$ & $L_{\text {Dmin }}$ & $L_{\text {CoCmax }}$ \\
\hline NML (mV) & $47-14.5=32.5$ & $46-13=33$ & $43-13.3=29.7$ & $44-13=31$ \\
\hline NMH (mV) & $181-96=85$ & $186-83=83$ & $185-80=85$ & $186-83=83$ \\
\hline SNM (mV) & 32.5 & $33(1.5 \%)$ & $29.7(-8.6 \%)$ & $31(-4.6 \%)$ \\
\hline E (aJ) & 807.7 & $843.4(4.4 \%)$ & $846.3(4.8 \%)$ & $821(1.7 \%)$ \\
\hline f(KHz) & 491 & $932.2(89.8 \%)$ & $887.6(80.8 \%)$ & $908(84.9 \%)$ \\
\hline
\end{tabular}




\subsection{Primitive and Complex Logic Gates}

In Sections 5.1 to 5.3 we studied the effect of the channel length on the delay and frequency optimization in simple circuits consisting of inverters. We noticed that in comparison to the commonly used minimum-size sub-threshold circuits, incorporating $L_{\text {CoCmax }}$ shows a drastic improvement in the performance for a small cost in the energy and SNM. In this section we perform a comparative study of using $L_{\text {CoCmax }}$ to RO circuits consisting of 2-input NAND (NAND2), 3-input NAND (NAND3), 2-input NOR (NOR2), and AND-OR-INVERTER (AOI) logic gates.

Although the results in Sections 5.2 and 5.3 show a good match between the delay optimization through simulations and that obtained by using $L_{\text {CoCmax }}$ from Section 5.1, parameter $L_{\text {CoCmax }}$ presented in Section 5.1 might not be a suitable choice to be used for complex gates. In complex gates, there are serial and parallel transistor connections, while in an inverter there is only one PMOS transistor in the pull-up network and one NMOS transistor in the pull-down network. Therefore, parameter $L_{\text {CoCmax }}$ should be extracted for the serial and parallel combination of transistors to be used in different logic gates.

For instances, a NAND2 logic gate consist of a stack of two NMOS transistors (in series) and two PMOS transistors connected in parallel. When studying a RO with NAND2 logic gates, one should study the worst-case scenario. In the worst-case scenario one of the PMOS transistors in the pull-up network should be kept in the "off" state and the NMOS transistor connected to the output should be kept in the "on" state, as shown in Figure 5.9.

To find $L_{\mathrm{CoCmax}}$ for a NAND2 logic gate, one should perform the COC simulation on configurations shown in Figure 5.10. For a stack of two NFETs, a test bench as shown in

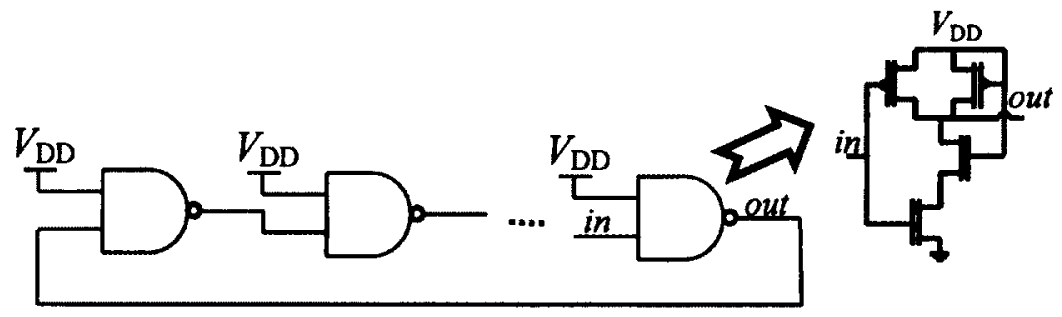

Figure 5.9 A RO with NAND2 logic gates connected in its worst-case scenario. 
Figure 5.10 (a) is used and $L_{\mathrm{CoCmax}}$ for this combination is found by maximizing $\frac{I}{C}=$ $\frac{1}{C_{G G N 1}+C_{D B N 2}}$ for two sets of input and output voltages as described in Section $5.1^{3}$. Similarly, $\frac{I}{C}=\frac{I}{C_{G G P 1}+C_{D B P 1}+C_{D B P 2}}$ is maximized to find $L_{C O C \max }$ for two PFETs connected in parallel as shown in Figure $5.10(\mathrm{~b}){ }^{4}$.

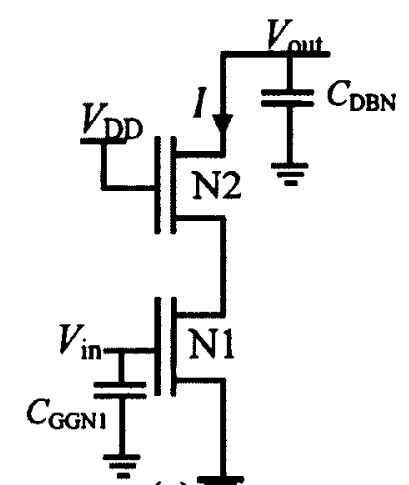

(a)

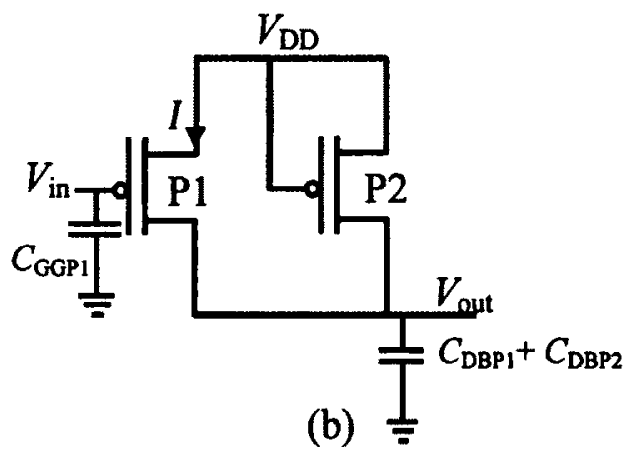

$$
\frac{I}{C}=\frac{I}{C_{G G N 1}+C_{D B N 2}} \quad \frac{I}{C}=\frac{I}{C_{G G P 1}+C_{D B P 1}+C_{D B P 2}}
$$

Figure 5.10 Pull-down (a) and pull-up (b) networks for a NAND2 logic gate connected in the worst case scenario.

Table 5.6 lists the results of simulations for these two configurations in each of the four technologies operating at $V_{\mathrm{DD}}=0.2 \mathrm{~V}$. The same procedure is applied to other usual combinations of transistors, i.e., three NFETs and three PFETs serial and parallel connections. The corresponding results are presented in Table 5.7.

Table $5.6 L_{C 0 C \max }$ for transistor configuration shown in Figure 5.10 at $V_{\mathrm{DD}}=0.2 \mathrm{~V}$.

\begin{tabular}{|c|c|c|c|c|}
\hline \multirow{2}{*}{$\begin{array}{c}\text { Technology node } \\
\text { Configuration }\end{array}$} & $180 \mathrm{~nm}$ & $130 \mathrm{~nm}$ & $90 \mathrm{~nm}$ & $65 \mathrm{~nm}$ \\
\cline { 2 - 5 } & \multicolumn{4}{|c|}{$L_{\text {CoCmax }}(\mathrm{nm})$} \\
\hline Two serial NFETs & 180 & 430 & 100 & 160 \\
\hline Two parallel PFETs & 280 & 380 & 240 & 210 \\
\hline
\end{tabular}

${ }^{3}$ The two sets are: 1) $V_{\text {in }}=V_{\mathrm{DD}} / 2, V_{\text {out }}=V_{\mathrm{DD}}$

${ }^{4}$ The two sets are: 1) $V_{\mathrm{in}}=V_{\mathrm{Do}} / 2, V_{\text {out }}=0$

2) $V_{\text {in }}=V_{\mathrm{DD}}, V_{\text {out }}=V_{\mathrm{DD}} / 2$

2) $V_{\text {in }}=0, V_{\text {out }}=V_{\mathrm{DD}} / 2$ 
Table 5.7 $L_{C O C \max }$ for different combinations of MOSFETs at $V_{\mathrm{DD}}=0.2 \mathrm{~V}$.

\begin{tabular}{|l|c|c|c|c|}
\hline \multirow{2}{*}{ Technology } & $180 \mathrm{~nm}$ & $130 \mathrm{~nm}$ & $90 \mathrm{~nm}$ & $65 \mathrm{~nm}$ \\
\cline { 2 - 5 } & \multicolumn{4}{|c|}{$L_{\text {CoCmax }}(\mathrm{nm})$} \\
\hline 1 PFET ( Section 5.1) & 270 & 320 & 240 & 170 \\
\hline 1 NFET ( Section 5.1) & 180 & 430 & 100 & 145 \\
\hline 2 Parallel PFETs & 280 & 380 & 240 & 210 \\
\hline 2 Parallel NFETs & 180 & 490 & 100 & 160 \\
\hline 3 Parallel PFETs & 290 & 400 & 240 & 230 \\
\hline 3 Parallel NFETs & 180 & 500 & 100 & 175 \\
\hline 2 Serial PFETs & 290 & 320 & 240 & 200 \\
\hline 2 Serial NFETs & 180 & 430 & 100 & 160 \\
\hline 3 serial PFETs & 290 & 330 & 240 & 200 \\
\hline 3 Serial NFETs & 180 & 440 & 100 & 170 \\
\hline
\end{tabular}

Depending on the topology of a logic gate, we incorporate $L_{\mathrm{COC} \max }$ of the appropriate configuration model to minimize its delay. For example, for NAND3, one should use $L_{\text {CoCmax }}$ for three Parallel PFETs and Stack of three serial NFETs from Table 5.7.

In Sections 5.2 and 5.3, the effectiveness of using $L_{\mathrm{CoCmax}}$ obtained from the $\mathrm{CoC}$ simulation for one NMOS and one PMOS transistor was verified. To verify the effectiveness of the new sets of channel lengths presented in Table 5.7, we performed simulations for ROs consisting of NAND2, NAND3, NOR2, and AOI. Table 5.8 shows the simulation results for ROs consisting of 29 of each logic gate operating at $V_{\mathrm{DD}}=0.2 \mathrm{~V}$. The obtained frequency by using $L_{\text {fmax }}$ (through simulation) and $L_{C o C \max }$ completely match in all four considered technologies. However, using $L_{\text {CoCmax }}$ instead of $L_{\text {fmax }}$ increases the energy consumption significantly in the $130 \mathrm{~nm}$ technology. For instance, using $L_{\text {CoCmax }}$ in a NAND3 RO increases the frequency by $99.7 \%$ for the cost of $23.5 \%$ increase in the energy consumption, while using $L_{\text {fmax }}$ improves the frequency by $118 \%$ and decreases the energy consumption by $4.4 \%$. This difference is even worse for the AOI RO. These large differences in the $130 \mathrm{~nm}$ technology is initiated from the NMOS transistor's strength compared to the PMOS transistor, which is not the case for other considered CMOS technologies. Only in this technology, $L_{\mathrm{COCmax}}$ for the PMOS transistor 
is smaller than that of the NMOS transistor. Since the NMOS transistor is inherently stronger than the PMOS transistor, making the channel length of the NMOS transistor longer than that of the PMOS transistor causes a slow transition from the "off" state to the "on" state and vice versa. This means more short-circuit current and, hence, more short-circuit energy consumption.

The last four right columns in Table 5.8 shows the result of simulations for ROs consisting of complex logic gates, but using $L_{\text {CoCmax }}$ obtained from the CoC method presented in Section 5.1. These results show a good match with the results obtained from incorporating $L_{\text {CoCmax }}$ of Table 5.7. In the $90 \mathrm{~nm}$ technology nodes $L_{\text {CoCmax }}$ for the PMOS transistor is always $240 \mathrm{~nm}$ and for the NMOS transistor is $100 \mathrm{~nm}$, regardless of the transistor connections and their count ${ }^{5}$. In the other three technologies, using $L_{C o C \max }$ from Table 5.7 results in higher frequencies than the frequencies obtained by using onetransistor $L_{C o C \max }$. This means that to have a frequency closer to $f_{\max }$ obtained by simulation, one should use $L_{\mathrm{COCmax}}$ depending on the gate topology.

Similar to the case of $L_{\mathrm{COC} \max }$ obtained for one transistor in Section 5.1, $L_{\mathrm{COC} \max }$ for the other configurations also depends on the supply voltage. Figure 5.11 shows this dependency to the supply voltage for different types of transistor connections that are typically used as building blocks in digital logic gates. In the $180 \mathrm{~nm}$ technology for the NMOS transistor, regardless of the connection topology and the number of transistors, $L_{\text {CoCmax }}$ occurs at $L_{\min }=180 \mathrm{~nm}$.

In Chapter 6, we present the results of using $L_{\mathrm{COC} \max }$ in a 32-bits CLA adder in the $65 \mathrm{~nm}$ technology to verify that which set of $L_{\mathrm{CoCmax}}$ shows more improvement in speed and energy consumption. Once, we used simple $L_{\mathrm{COC} \max }$ obtained in Section 5.1, then, we incorporated suitable $L_{\mathrm{CoCmax}}$ from Table 5.7(depending on the logic gate configuration).

\footnotetext{
${ }^{5}$ This might be due to the specific model used by the simulation CAD tool, Cadence
} 
Table 5.8 Simulation results for $\mathrm{RO}$ consisting of 29 of each logic gate for four sets of channel lengths at $V_{\mathrm{DD}}=0.2 \mathrm{~V}$.

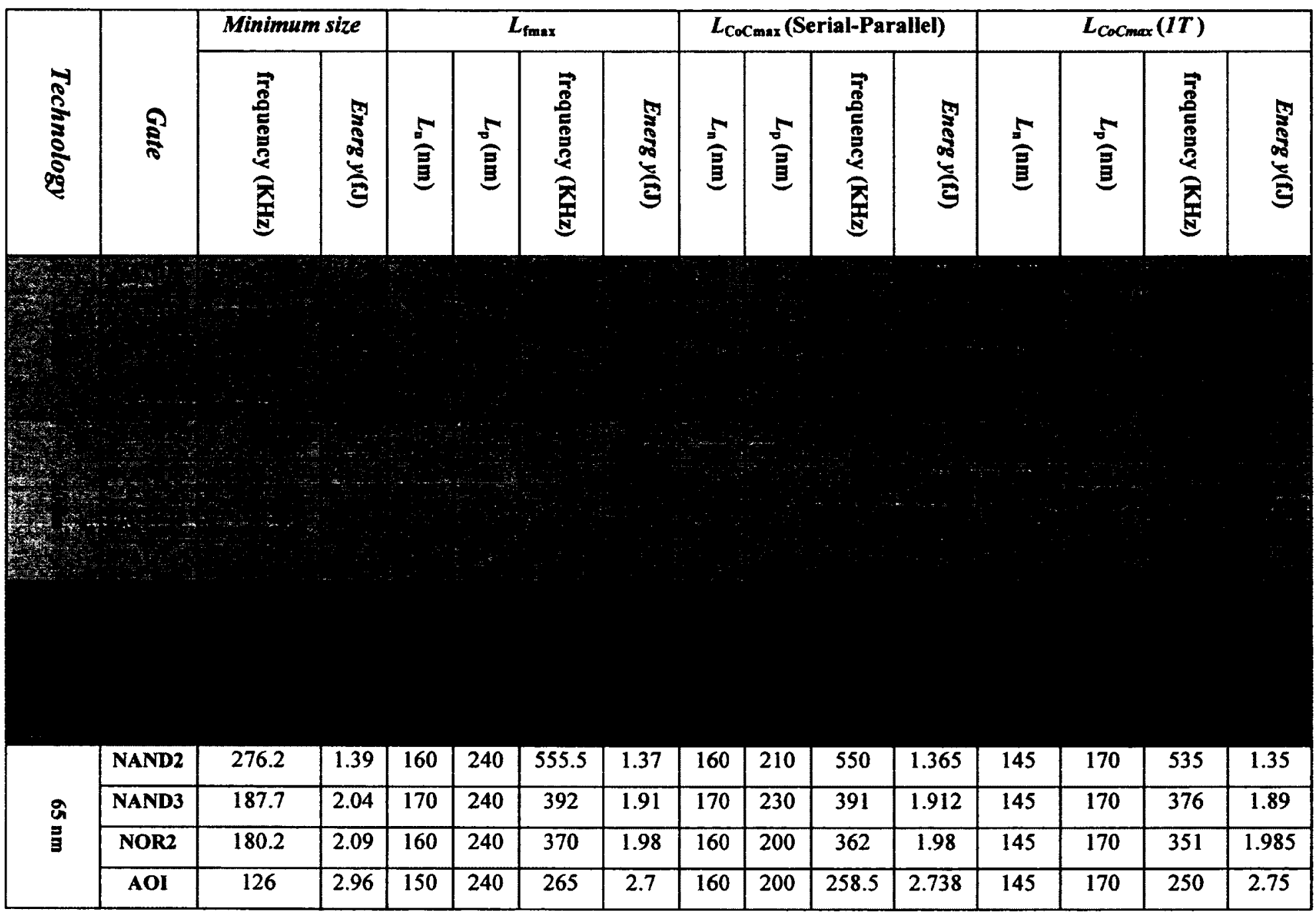



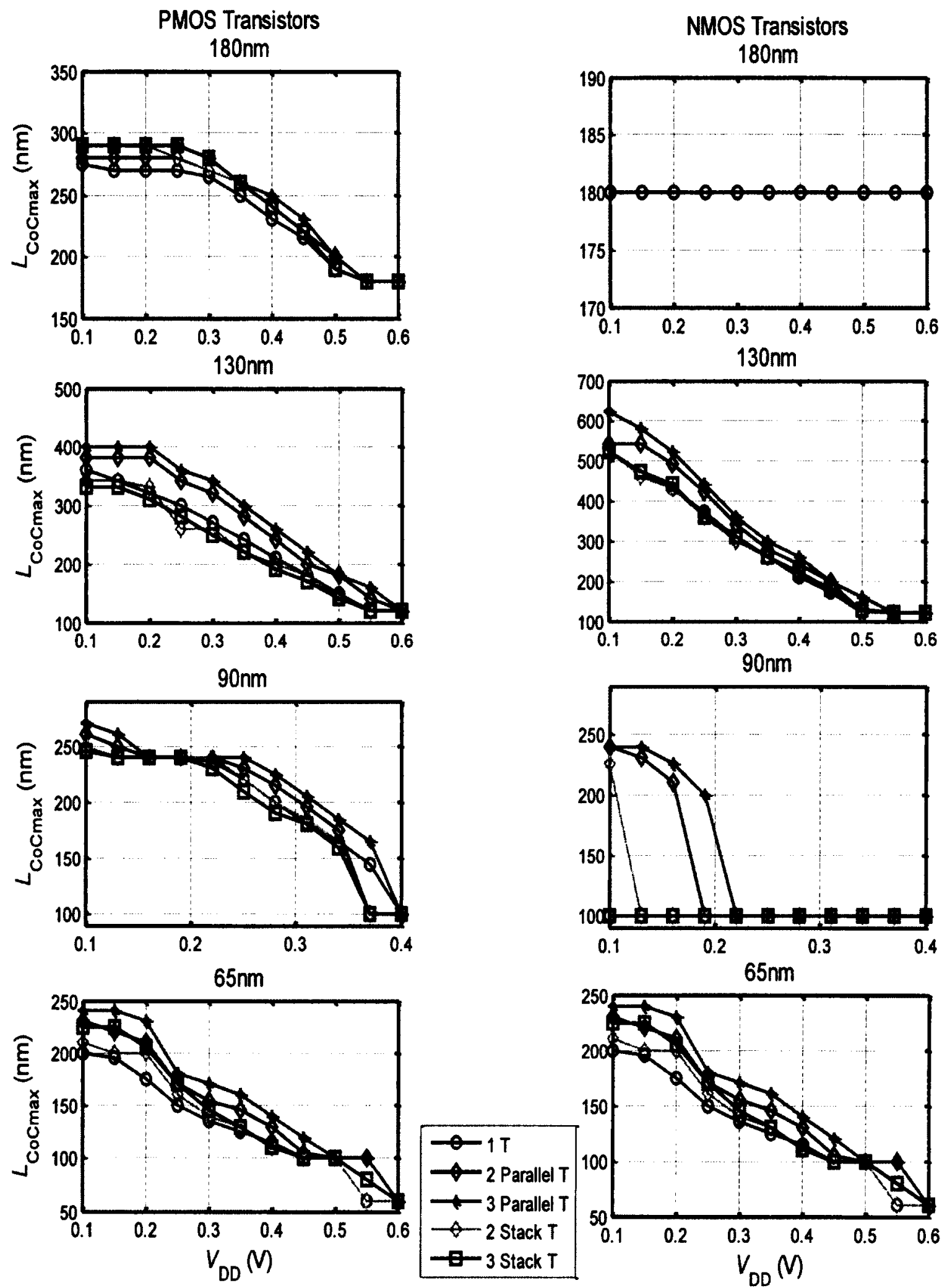

Figure $5.11 L_{C O C \max }$ versus $V_{\mathrm{DD}}$ for different connection topologies of transistors used as a building blocks for logic gates 


\subsection{Chapter Summary}

In this chapter we proposed a method to optimize the delay (or operation frequency) of a digital circuit operating in the sub-threshold region. Our proposed method is based on maximizing the $\mathrm{CoC}$ ratio by manipulating the channel lengths of transistors. First, we biased different typical pull-up and pull-down networks that are used as building blocks of digital gates, e.g., stack of three serial transistors or parallel connection of two transistors. Then, we plotted $\mathrm{CoC}$ versus the channel length to find $L_{\mathrm{COC} \max }$ for each type of transistors in the four considered technologies. For all different topologies and for all considered technology nodes, $L_{\mathrm{COC} \max }$ is extracted based on simulation.

Due to the dependency of $L_{\mathrm{COC} m a x}$ to the supply voltage and the logic gate configuration, one should decide to choose the correct $L_{C O C \max }$ to optimize the performance of the circuit. Although incorporating $L_{\mathrm{CoC} \max }$ does not result in the absolute maximum frequency, the frequency obtained by using $L_{\mathrm{COCO}_{\max }}$ is still within an acceptable margin from the maximum frequency for all considered technologies.

Besides to performance and energy consumption improvement, the $\mathrm{CoC}$ method is very time efficient in the delay optimizing for large digital circuits with numerous transistors. The CoC method is based on DC simulations and is much faster than the transient analysis that is used to find $L_{\mathrm{fmax}}$. The $\mathrm{CoC}$ method opens a perspective for the designers to choose a suitable starting point for simulation instead of doing blind exhaustive and time consuming simulations. 


\section{Implications and Applications}

In this chapter, we report the implications of our results and using the optimum channel lengths obtained in Chapter 5 in some applications.

\subsection{Increasing $V_{\mathrm{DD}}$ versus Channel-length Manipulation}

Verma et. al. in [64] claimed that using minimum-size transistors is the best choice for sub-threshold circuits. They showed that to decrease the propagation delay along the critical path, a slightly increase in the supply voltage from its minimum-energy point, is more efficient than the conventional gradual sizing. Figure 6.1 verifies that claim, where energy and frequency of a 29-INV RO is plotted versus $V_{D D}$ in the $65 \mathrm{~nm}$ technology for the minimum channel length. This plot shows two sets of simulation results, one for the

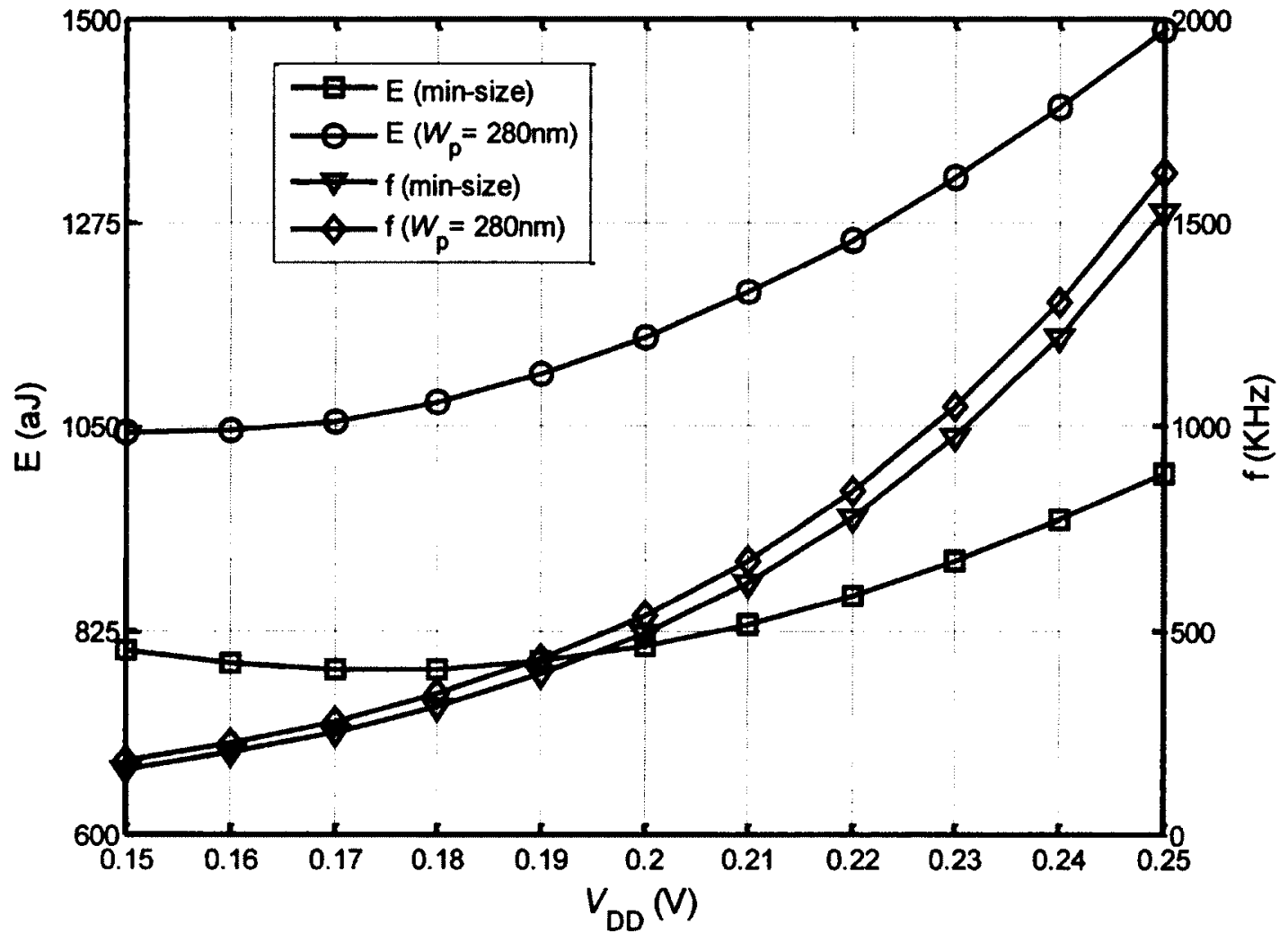

Figure 6.1 Energy per operation and frequency vs. $V_{D D}$ for a 29- INV RO in the $65 \mathrm{~nm}$ technology at $L_{\min }=60 \mathrm{~nm}$. 
minimum size and the other for the channel width that maximize the frequency (obtained by simulation). This plot shows that increasing the channel width, as it is the conventional method of sizing, increases the frequency slightly for a large cost in the energy consumption. Also, it shows that if $V_{\mathrm{DD}}$ increases from its minimum energy point, $0.17 \mathrm{~V}$, to $0.2 \mathrm{~V}$, energy is almost constant for about $100 \%$ increase in the frequency, while keeping the size of the transistors minimum. This implies that one can increase the supply voltage slightly from the minimum energy point to increase the performance rather than increasing the channel width.

However, increasing the channel length will affect the delay and energy completely differently from the way that the channel width does. As explained in Section 4.5, increasing the channel length decreases the sub-threshold slope that makes the transistors faster and shortens the short-circuit time. This leads to an improvement in the performance for a small penalty in the energy. Figure 6.2 shows the energy and frequency

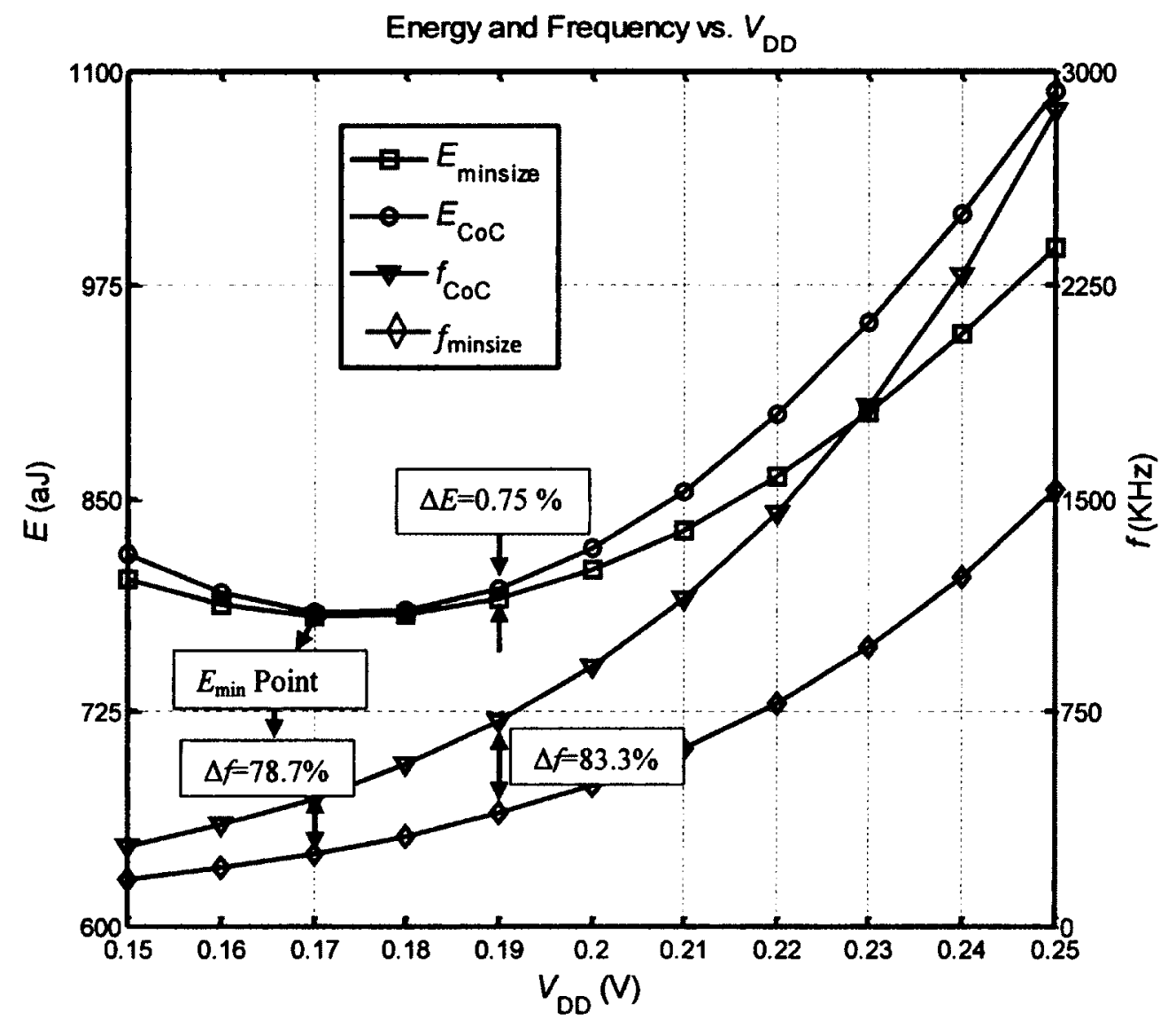

Figure 6.2 Energy per operation and frequency vs. $V_{D D}$ for a 29- INV $R O$ in the $65 \mathrm{~nm}$ technology at $W_{\min }=120 \mathrm{~nm}$. 
versus $V_{\mathrm{DD}}$ for a 29-INV RO simulated for two sets of transistor sizes in the $65 \mathrm{~nm}$ technology at $W_{\min }=120 \mathrm{~nm}$. Both sets show the same minimum-energy point located at $V_{\mathrm{DD}}=0.17 \mathrm{~V}$. Incorporating $L_{\mathrm{COC} \max }$ from Table 5.1 in the $\mathrm{RO}$ does not change the minimum energy, but increases the frequency by $78.7 \%$. Additionally, increasing the supply voltage from the minimum point to a larger value, e.g, $V_{D D}=0.19 \mathrm{~V}$, results in an $83.3 \%$ increase in the frequency for only a $0.75 \%$ cost in the energy. This plot shows that Verma's claim is not valid for improving the performance by up-sizing the channel length. For instance, if the up-sized circuit operates at $V_{\mathrm{DD}}=0.19 \mathrm{~V}$, the frequency of operation and energy are $750 \mathrm{KHz}$ and $725 \mathrm{aJ}$, respectively. Suppose that one is interested in exploiting the minimum-size circuit with increased supply voltage to achieve the same performance. The plot shows that $V_{\mathrm{DD}}$ should be increased to $0.22 \mathrm{~V}$. This results in an energy consumption of 863 aJ that is $10 \%$ above the energy consumption caused by increasing the channel length to $L_{\mathrm{COCmax}}$ operating at $V_{\mathrm{DD}}=0.19 \mathrm{~V}$.

Although in this research we are not discussing the energy minimizing in detail and it will be presented in the future work, it is worth to show a graph for more clarification on the effects of the channel length upsizing on the energy and performance of a circuit. Parameter $L_{\text {CoCmax }}$ presented in Table 5.1 does not necessarily result in the minimum energy. Simulations show that the channel lengths where the energy becomes minimum are longer than the minimum channel length and they differ from $L_{\text {CoCmax }}$. Using these new sets of channel lengths obtained by simulation in a $29-\mathrm{INV}$ RO, results in curves illustrated in Figure 6.3. It shows that increasing the channel length moves the minimumenergy point towards smaller supply voltages. In addition, using suitable channel lengths reduces the minimum energy value by $15.8 \%$ while the performance increases compared to the RO with minimum-size transistors.

Again, if we want to compare the effect of increasing $V_{\mathrm{DD}}$ and increasing the channel length, consider the case that the $\mathrm{RO}$ is upsized and operating in $V_{\mathrm{DD}}=0.2 \mathrm{~V}$. The frequency and energy are $625 \mathrm{KHz}$ and $740 \mathrm{aJ}$, respectively (point A). If we want to keep the minimum size and increase the $V_{\mathrm{DD}}$ to have the same performance as point $\mathrm{A}$, we have to increase $V_{\mathrm{DD}}$ to $0.21 \mathrm{~V}$ (point $\mathrm{B}$ ). At this point the energy is $830 \mathrm{aJ}$ that is $12.2 \%$ more than upsized circuit operating at the lower voltage. 


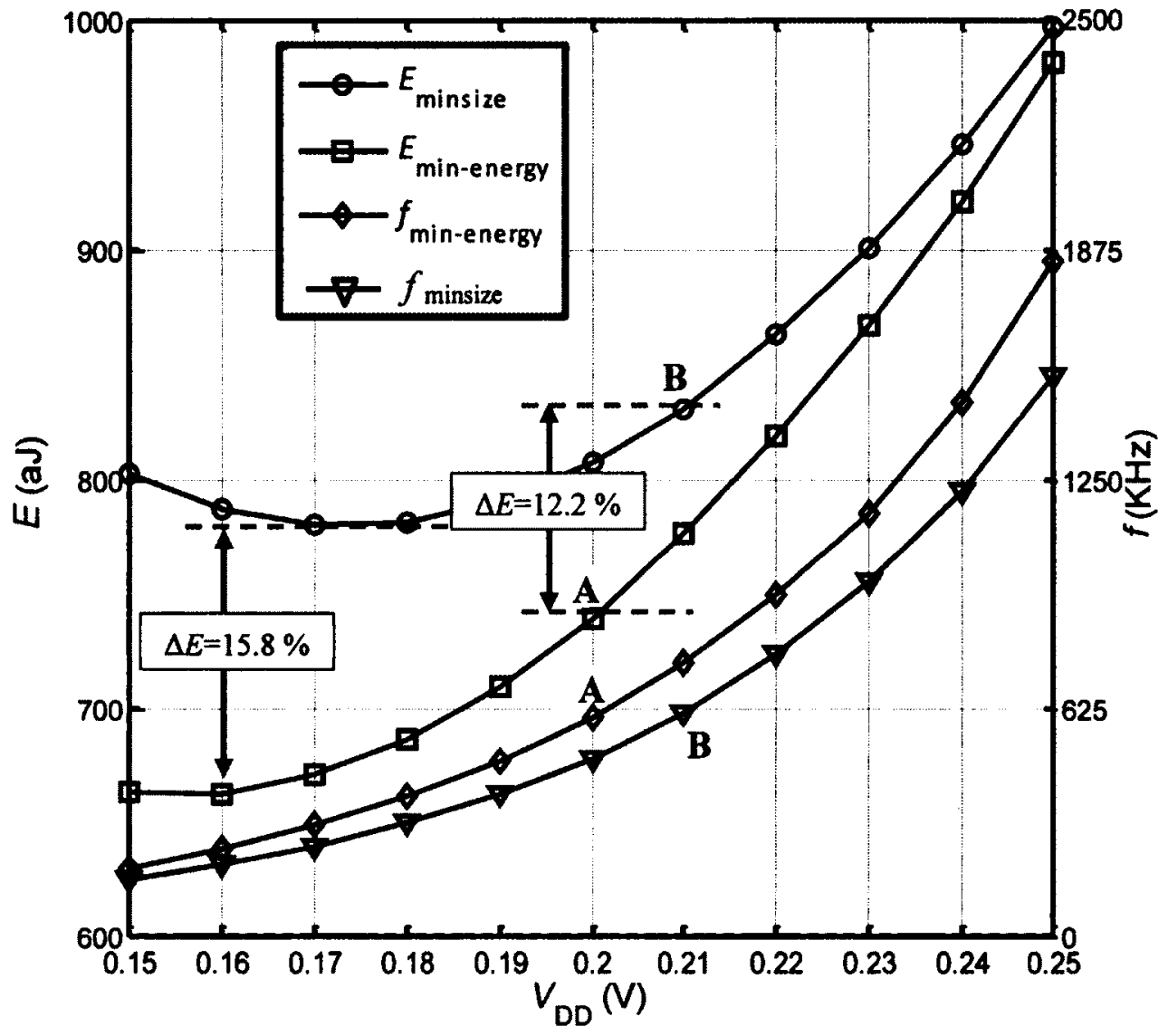

Figure 6.3 Energy per operation and frequency vs. $V_{D D}$ for a 29- INV $R O$ in the $65 \mathrm{~nm}$ technology at $W_{\min }=120 \mathrm{~nm}$.

Figure 6.4 depicts EDP curves versus $V_{\mathrm{DD}}$ for four different sets of transistor sizes. It shows that using $L_{\mathrm{COCmax}}$ and channel lengths resulting in the minimum energy leads to smaller EDPs compare to circuits with minimum-size transistors or wider PMOS transistors. Using $L_{\text {COCmax }}$ results in the smallest EDP values.

\subsection{2-bit CLA Adder}

The adder consists of eight similar blocks. Each block adds two 4-bit sets of the inputs. For example, the first block takes $\mathrm{A}[1: 4]$ and $\mathrm{B}[1: 4]$ and $\mathrm{Cin}$ as its inputs and produces $S[1: 4]$ and Cout as the outputs. Cout of the first block is fed to the next block and summation is performed on this input and the next 4-bits of $A$ and $B(A[5: 8]$ and $\mathrm{B}[5: 8])$. The block diagram of a 4-bit CLA adder is shown in Figure 6.5. Group PG 


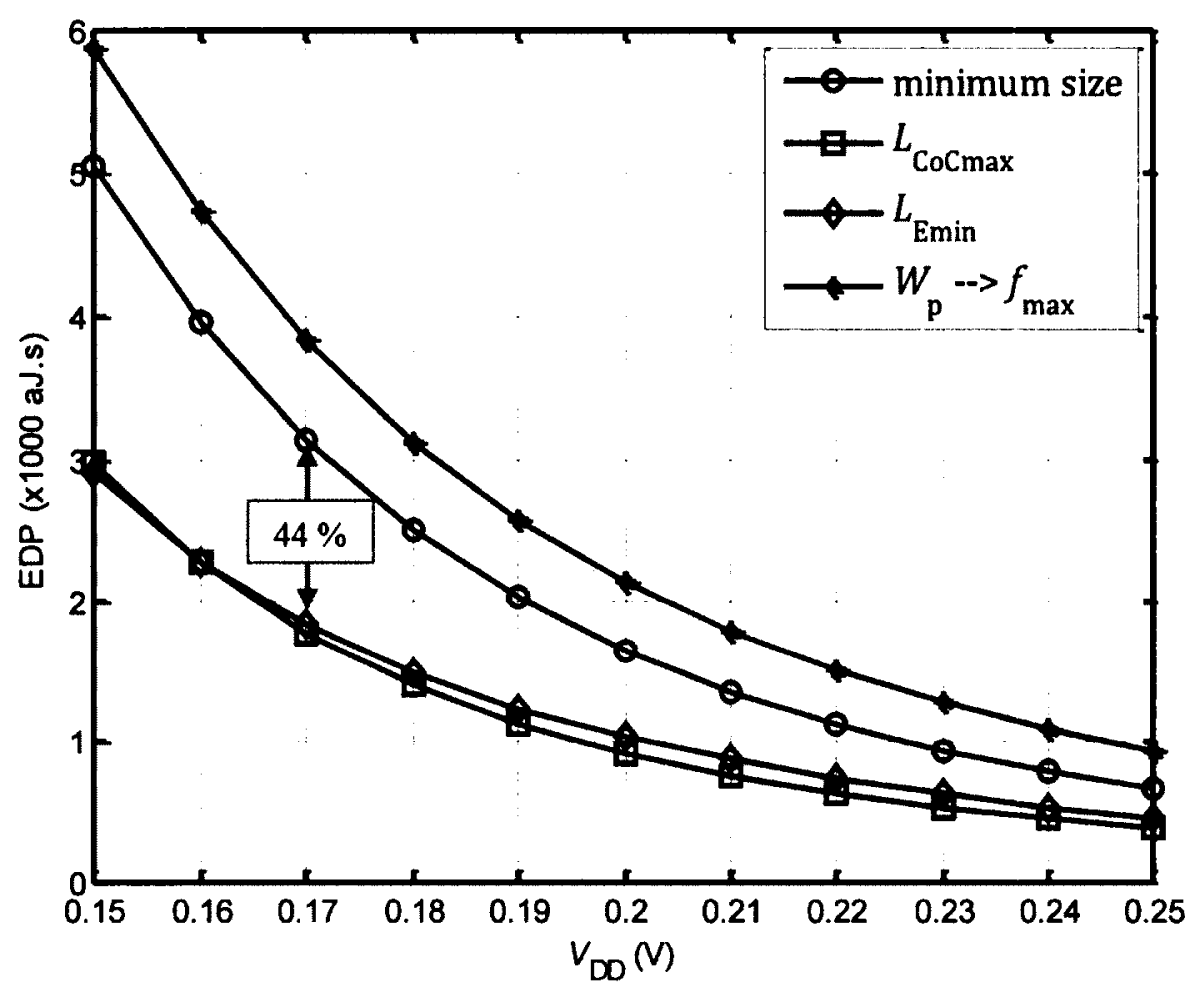

Figure 6.4 EDP versus $V_{D D}$ for a 29-INV RO simulated for four different sets of transistor sizes in the $65 \mathrm{~nm}$ technology.

consists of four bitwise propagate-generate (PG) units. Bitwise PGs use A and B inputs to evaluate $P$ and $G$ signals. The bitwise $P G$ signals are combined with Cin to produce the four output bits of the sum, S[1:4]. The bitwise PG signals are also used to generate the group PG signals. A combination of the group PG signals with Cin generates Cout [15].

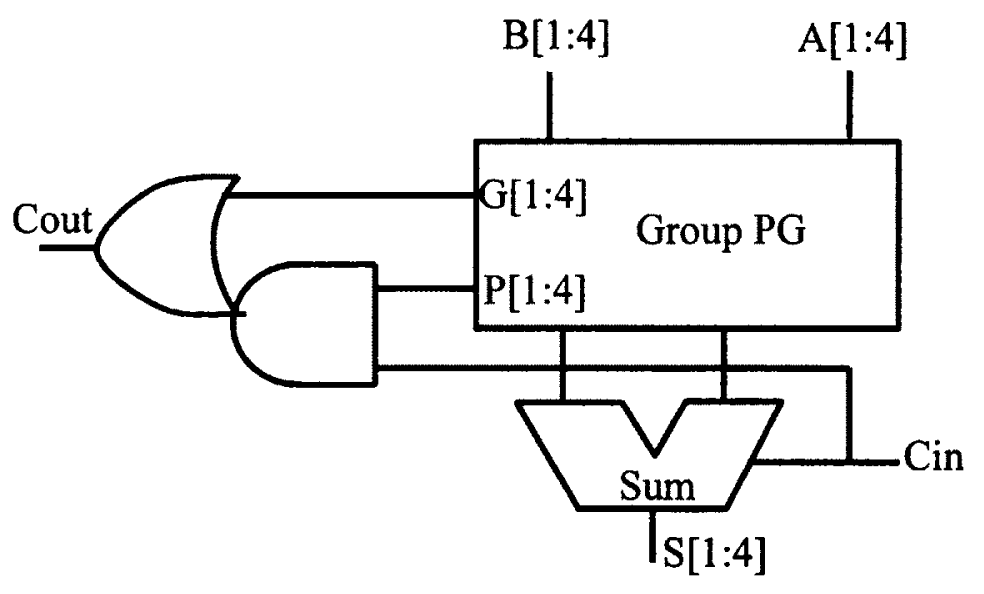

Figure 6.5 Block diagram for a 4-bit CLA adder [15]. 
Each bitwise PG group consists of four NAND2 and three INV. A sum block consists of 15 NAND2, $14 \mathrm{INV}$, and three NOR2. The group PG block has five NAND2, four NOR2 and six INV. Hence, total adder has 192 NAND2, 56 NOR2, and 184 INVs. We simulated the adder with three different sets of sizings. The first is an adder with minimum-size transistors. In the second adder, the channel lengths are set to $L_{C O C \max }$ presented in Table 5.1. And finally, the channel lengths for parallel and serial combinations presented in Table 5.7 are incorporated in the adder as the third set. The area of the first adder is $1869 \mu \mathrm{m}^{2}$ [6]. Using $L_{\mathrm{CoCmax}}$ from Table 5.1 and Table 5.7 in the adder circuit, increases the area of the adder to $4900 \mu \mathrm{m}^{2}$ (162\% increase compared to minimum-size adder) and $5390 \mu \mathrm{m}^{2}$ (188\% increase compared to minimum-size adder), respectively. The channel width is fixed at its minimum value, $W_{\min }=120 \mathrm{~nm}$.

Table 6.1 shows the results of simulations. Using both sets of $L_{\text {CoCmax }}$ improves the delay, energy per operation, and EDP at the same time. None of these quality metrics are degraded, similar to what happened for the ROs studied in Chapter 5 . Using $L_{\text {CoCmax }}$ in the adder results in about $50 \%, 20 \%$, and $60 \%$ improvements in the delay, energy, and EDP, respectively. Incorporating $L_{\mathrm{CoCmax}}$ from Table 5.7 improves the delay by $5 \%$ compared to using $L_{\mathrm{CoCmax}}$ from Table 5.1 for a cost of $20 \%$ increase in the area of the adder.

Table 6.1 Simulation results for a 32 -bit CLA adder in the $65 \mathrm{~nm}$ technology at $V_{\mathrm{DD}}=0.2$.

\begin{tabular}{|c|c|c|c|c|c|c|c|c|c|}
\hline & \multicolumn{3}{|c|}{ Minimum Size } & \multicolumn{3}{|c|}{$L_{\text {CoCmax }}($ Table 5.1) } & \multicolumn{3}{|c|}{$L_{\text {CoCmax }}($ Table 5.7) } \\
\hline Test Case & $\begin{array}{c}t_{p} \\
\text { (ns) }\end{array}$ & $\begin{array}{c}E \\
\text { (fJ) }\end{array}$ & $\begin{array}{c}\text { EDP } \\
\left(\mathbf{x 1 0}^{-21} \mathbf{3} .5\right)\end{array}$ & $\begin{array}{c}t_{p} \\
\text { (ns) }\end{array}$ & $\begin{array}{c}E \\
\text { (fJ) }\end{array}$ & $\begin{array}{c}\text { EDP } \\
\left.\left(\mathbf{x} 10^{-21}\right\lrcorner .5\right)\end{array}$ & $\begin{array}{c}t_{p} \\
(n s)\end{array}$ & $\begin{array}{c}E \\
\text { (fJ) }\end{array}$ & $\begin{array}{c}\text { EDP } \\
\left(\times 10^{-21} J .5\right)\end{array}$ \\
\hline $\begin{array}{l}\mathrm{A}=0, \mathrm{~B}=1 \\
\text { Cin toggles }\end{array}$ & 2569 & 7.12 & 18.29 & 1310 & 5.69 & 7.45 & 1289 & 5.67 & 7.3 \\
\hline $\begin{array}{l}A, B, C \text { Cin } \\
\text { toggle the } \\
\text { same }\end{array}$ & 425 & 1.2 & 0.51 & 226 & 1.03 & 0.233 & 220 & 1 & 0.22 \\
\hline $\begin{array}{l}A=1, B \& C \text { in } \\
\text { toggle the } \\
\text { same }\end{array}$ & 449 & 1.47 & 0.66 & 235 & 1.14 & 0.27 & 228 & 1.2 & 0.27 \\
\hline random & 2780 & 8.25 & 22.93 & 1410 & 6.45 & 9.1 & 1385 & 6.42 & 8.9 \\
\hline
\end{tabular}




\subsection{Driving Large-Loads}

Verma's claim even becomes worse when a circuit drives large loads. Consider the situation that a chain of inverters are driving an inverter 64 times larger than the minimum-size inverter (representing a 64-bits data line). In the logical effort method [15], it is shown that for driving a $64 \mathrm{X}$ inverter, the optimum number of stages is three and the optimum tapering factor is $\sqrt[3]{64}=4$. It means that to have the minimum delay in driving a $64 \mathrm{X}$ inverter (load), the inverters in the chain should have channel widths one, four, and 16 times wider than the minimum channel width. Since in this thesis we are studying the channel length manipulation, we keep the channel width of transistors in their minimum value. Two cases are studied to compare the effects of increasing the supply voltage and upsizing the channel length. In the first case all inverters are minimum size and in the second case the channel lengths are upsized to $L_{\text {CoCmax }}$ in Table 5.1 for the $65 \mathrm{~nm}$ technology. A square-wave signal is applied to the input node and the propagation delays are measured between the output and input nodes shown in Figure 6.6. Table 6.2 shows the results of simulation. The first row shows the delay, power, and energy consumption for the chain with minimum-size inverters. The second row is the measurement results when $L_{\mathrm{COCmax}}$ is used in the inverters of the chain. $P_{\mathrm{ch}}$ and $E_{\mathrm{ch}}$ refer to the power and energy consumption in three inverters in the chain and $P_{\mathrm{L}}$ and $E_{\mathrm{L}}$ refer to the power and energy consumption in the load. The table shows that incorporating $L_{\text {CoCmax }}$ results in a $100 \%$ increase in the operation frequency ( $~ 50 \%$ reduction in delay). Total energy consumption shows $11.6 \%$ reduction after using $L_{\text {CoCmax }}$ in the inverters of the chain.

Table 6.2 Simulation results for a three-inverters chain driving a large load at $V_{\mathrm{DD}}=0.2 \mathrm{~V}$.

\begin{tabular}{|c|c|c|c|c|c|c|c|c|c|}
\hline & $\begin{array}{c}t_{\mathrm{plh}} \\
\mathrm{ns}\end{array}$ & $\begin{array}{c}t_{\mathrm{phl}} \\
\mathrm{ns}\end{array}$ & $\begin{array}{c}t_{\mathrm{p}} \\
\mathrm{ns}\end{array}$ & $\begin{array}{c}\mathrm{f}_{\max } \\
\mathrm{KHz}\end{array}$ & $\begin{array}{c}P_{\mathrm{ch}} \\
\mathrm{pW}\end{array}$ & $\begin{array}{c}E_{\mathrm{ch}} \\
\mathrm{aJ}\end{array}$ & $\begin{array}{c}P_{\mathrm{L}} \\
\mathrm{pW}\end{array}$ & $\begin{array}{c}E_{\mathrm{L}} \\
\mathrm{aJ}\end{array}$ & $\begin{array}{c}E_{\text {total }} \\
\mathrm{aJ}\end{array}$ \\
\hline Min-size & 673 & 167 & 420 & 250 & 133 & 531 & 177 & 710 & 1241 \\
\hline \multirow{2}{*}{$L_{\text {CoCmax }}$} & \multirow{2}{*}{355} & \multirow{2}{*}{83} & 218.5 & 500 & \multirow{2}{*}{261} & 521 & 288 & 576 & 1097 \\
& & & $-48 \%$ & $100 \%$ & & & & $-11.6 \%$ \\
\hline
\end{tabular}




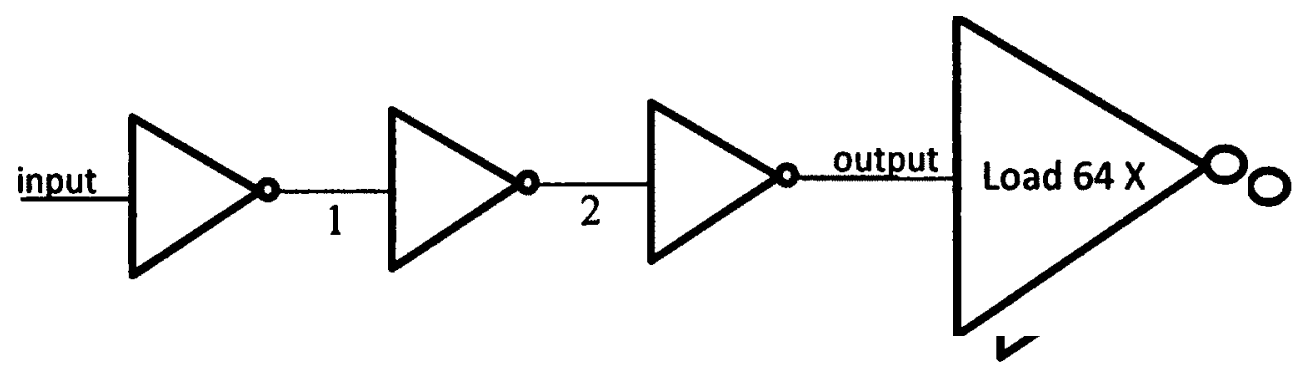

Figure 6.6 Driving a large load with a chain of three inverters.

Incorporating $L_{\mathrm{COCmax}}$ in inverters leads to faster inverters and sharpens the rising and falling edge of the signal at the output node as depicted in Figure 6.7. This results in a shorter time of short-circuit current. Increasing the channel length, decreases both $E_{\mathrm{ch}}$ and $E_{\mathrm{L}}$. If we want to use the minimum-size inverters in the chain and increase $V_{\mathrm{DD}}$ to have the same performance as using upsized inverters, $V_{\mathrm{DD}}$ should increase to $0.225 \mathrm{~V}$ while the supply voltage of load is not changed. Using the new supply voltage results in

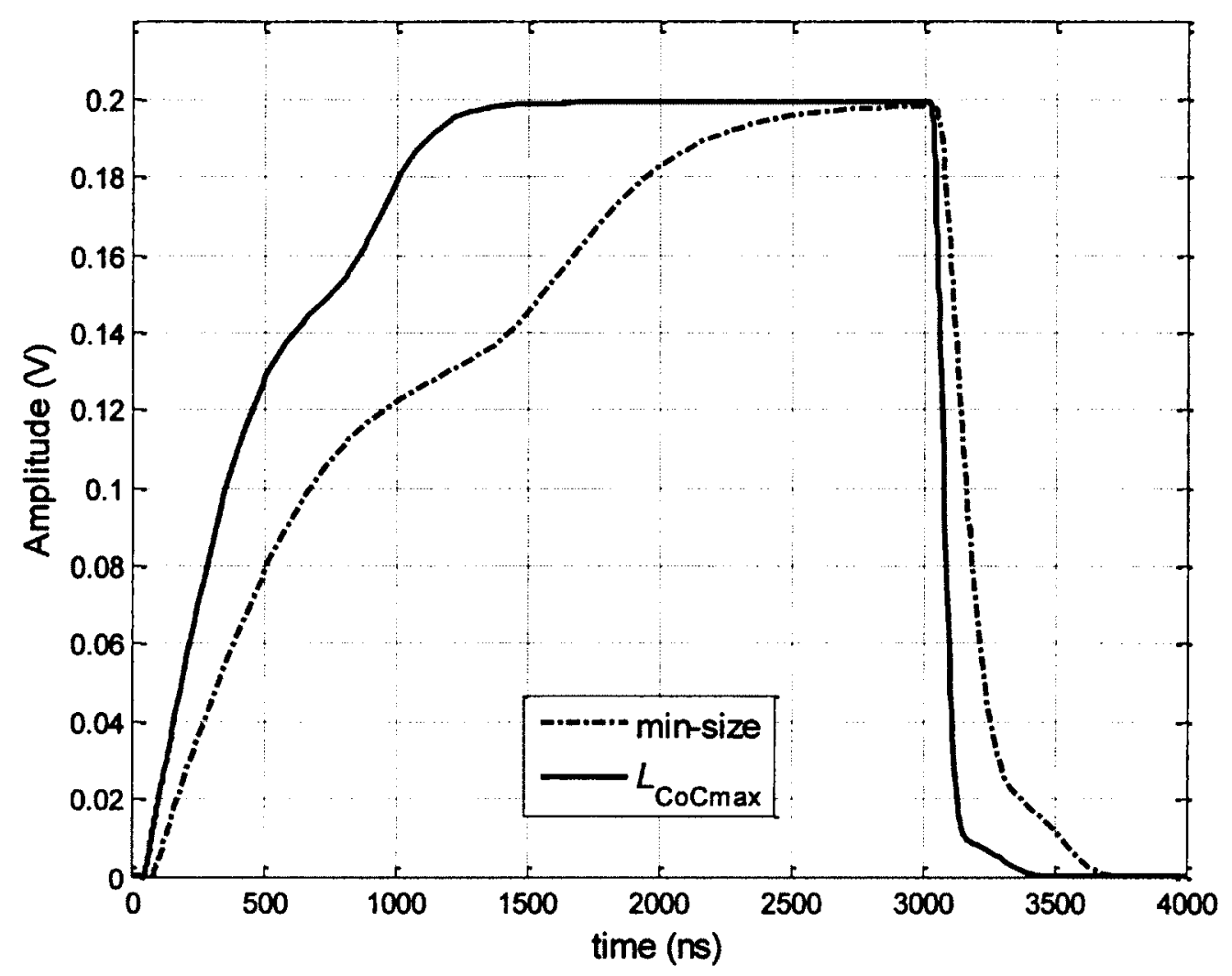

Figure 6.7 Output signal of a chain driving a large load at $V_{D D}=0.2 \mathrm{~V}$ and $W_{\min }=120 \mathrm{~nm}$ in the $65 \mathrm{~nm}$ technology. 
$1186 \mathrm{aJ}$ total energy consumption energy, which is $7 \%$ more than of the chain with upsized inverters and lower supply voltages.

In Figure 6.6 there is no off-path logic gates connected to the intermediate nodes along the critical path. In practical cases, in each node of the critical path there are usually some off-path connections. We repeated the same simulation as done before for Figure 6.6, except that in nodes 1 and 2 we connected three other minimum-size inverters in parallel. These inverters add loading effects to the nodes along the critical path. In these kinds of circuits that there are some off-path connections along the critical path, increasing the channel length is even more beneficial than increasing the supply voltage. the results are reported in Table 6.3. to have a minim-size chain performing as the upsized chain, one should increase the supply voltage to $227 \mathrm{mV}$ that results in a total energy of $1377 \mathrm{aJ}$, which is $9 \%$ more than of upsized chain operating at lower $V_{\mathrm{DD}}$.

Table 6.3 Simulation results for a three-inverters chain driving a large load at $V_{\mathrm{DD}}=0.2 \mathrm{~V}$. In each intermediate node three minimum-size inverters are connected in parallel as offpath logic gates.

\begin{tabular}{|c|c|c|c|c|c|c|c|c|c|}
\hline & $\begin{array}{c}t_{\text {plh }} \\
\mathrm{ns}\end{array}$ & $\begin{array}{c}t_{\text {phl }} \\
\mathrm{ns}\end{array}$ & $\begin{array}{c}t_{\mathrm{p}} \\
\mathrm{ns}\end{array}$ & $\begin{array}{c}\mathrm{f}_{\max } \\
\mathrm{KHz}\end{array}$ & $\begin{array}{c}P_{\mathrm{ch}} \\
\mathrm{pW}\end{array}$ & $\begin{array}{c}E_{\mathrm{ch}} \\
\mathrm{aJ}\end{array}$ & $\begin{array}{c}P_{\mathrm{L}} \\
\mathrm{pW}\end{array}$ & $\begin{array}{c}E_{\mathrm{L}} \\
\mathrm{aJ}\end{array}$ & $\begin{array}{c}E_{\text {total }} \\
\mathrm{aJ}\end{array}$ \\
\hline Min-size & 749 & 247 & 498 & 250 & 144 & 574 & 200 & 800 & 1374 \\
\hline \multirow{2}{*}{$L_{\text {CoCmax }}$} & \multirow{2}{*}{387} & \multirow{2}{*}{114} & 250.5 & 487 & 283 & 581 & 330 & 676 & 1257 \\
& & & $-49.6 \%$ & $94.8 \%$ & & & $-8.5 \%$ \\
\hline
\end{tabular}

\subsection{Chapter Summary}

Using $L_{\mathrm{COC} \max }$ in the sub-threshold circuits reduces the energy consumption in parallel to the improvement of the speed of circuit. This fact shows that the minimum energy operation is not always associated with using minimum-size transistors in circuits and increasing the supply voltage to compensate the lower speed, which is a popular belief. 


\section{Conclusion}

This chapter summarizes the research work' contributions of this thesis, and proposed future work, which will help researchers to advance the art of sub-threshold design for next generation of electronic devices.

The rapidly growing portable-electronics market as well as thermal dissipation has launched a massive trend towards low-power and low-voltage design techniques. Among these techniques, the sub-threshold offers the minimum energy consumption for the cost of speed, which is still acceptable for some ULP applications such as micro-sensor networks and biomedical devices. Nevertheless, a number of research projects have been established on increasing the speed of sub-threshold circuits to extend applications of these kinds of circuits to relatively higher frequencies. Increasing the width of transistors is the most common method to increase the speed of a digital circuit, besides increasing $V_{\mathrm{DD}}$. In the sub-threshold region due to the INWE, manipulating the channel width needs special consideration. The literature has been addressing the concept of manipulating the channel width of transistors in sub-threshold circuits. However, manipulating the channel length is not common in the super-threshold region, and in the sub-threshold region is a very new topic. Changing the channel length affects different characteristics of a transistor such as the threshold voltage, current, capacitance, and sub-threshold slope. Each of these has its effect on the delay and energy consumption. In this thesis these effects are studied in detail and a method for minimizing the delay in sub-threshold circuits is proposed based on channel length manipulations.

\subsection{Summary}

Unlike the super-threshold design, where the channel length is mostly fixed at its minimum, in the sub-threshold region the channel length can be increased to achieve a higher driving current. This implies a possibility for a maximum in the current curve versus the channel length. In the $180 \mathrm{~nm}$ technology, only the PMOS transistor shows a 
maximum in the current versus the cannel length and the current for the NMOS transistor is maximum at the minimum channel length. For the $130 \mathrm{~nm}$ and $90 \mathrm{~nm}$ technologies both types of transistors show a maximum in their current curves versus the channel length. In the $65 \mathrm{~nm}$, where two "lvt" and "svt" flavours are offered, both transistors of "svt" type show a descending behaviour with respect to the channel length, while transistors of "lvt" type have maximum point in their current curves versus the channel length.

Since the current has the main rule in the delay, it seems that maximizing the current minimizes the delay. But this is not correct, because of the capacitances dependence to the channel length. Since the delay is related to the ratio of capacitances over current, we studied $\mathrm{CoC}$ to find an optimal channel length minimizing the delay.

In this thesis, a new method of $\mathrm{CoC}$ measurement is introduced. Applying this method results in optimum channel lengths sets, $L_{\mathrm{CoCmax}}$. Using $L_{\mathrm{COC}_{\max }}$ in inverter ROs leads to delays and frequencies relatively close to the minimum delay and maximum frequency obtainable through simulations in Cadence. Although the $\mathrm{CoC}$ method is also based on simulation in Cadence, it is a DC simulation and is very fast compare to doing transient analysis to find the maximum frequency or minimum delay. Using $L_{C O C \max }$ in a 29-INV RO results in a frequency only $2.5 \%$ less than the frequency obtained through exhaustive simulations. Incorporating $L_{\mathrm{COC} \max }$ in a 29-INV RO improves the frequency up to $95 \%$ compared to the minimum-size RO in the $130 \mathrm{~nm}$ technology.

A test bench for different combinations of transistor connections are also introduced in the thesis. Digital logic gates usually contain two or three transistors connected in series or parallel. Hence, we introduced new test-benches to find the optimum channel lengths for different configurations. Depending on the topology of a logic gate, one can decide to use appropriate channel lengths.

The $\mathrm{CoC}$ method shows its effectiveness when one wants to find the maximum frequency for a large circuit with many transistors. For instance, a 32-bit CLA adder has 1360 transistors. Performing transient simulation to find the optimal channel lengths is almost impossible. Using $L_{\mathrm{CoCmax}}$ in a 32-bit CLA adder results in a $50 \%$ improvement in the delay in the $65 \mathrm{~nm}$ technology. 
In addition, increasing the channel length improves the sub-threshold slope of a transistor. Improved sub-threshold slope results in faster transistors with lower shortcircuit energy consumption. In most cases using $L_{\text {CoCmax }}$ results in a lower energy consumption compared to the minimum-size circuit. For example, in a 32-bit CLA adder, incorporating $L_{\mathrm{COC} \max }$ decreases the energy consumption by $20 \%$ and improves the EDP by $60 \%$ compared to the minimum-size adder in the $65 \mathrm{~nm}$ technology.

\subsection{Contributions}

The following are the major contributions of this thesis.

1. Studying the effect of channel length manipulation on the current, capacitances and sub-threshold slope in detail.

2. Introducing a method for obtaining the optimal channel length to minimize the delay of a transistor.

3. Extending the above method to serial and parallel connections of transistors.

4. Introducing test benches and biasing techniques to find the optimal channel lengths.

5. Applying the method to simple and complex circuits to prove the concept.

6. Demonstrating that in contrast to the popular belief, the minimum energy operation is not always associated with minimum size transistors, and that it can be lowered by manipulating channel length.

7. Increasing the speed of sub-threshold circuits that leads to extending the application of these circuits to devices with relatively higher frequencies.

\subsection{Future work}

To continue the work presented in this thesis, the following research directions are proposed.

1. Deriving an analytical model for finding $L_{\mathrm{CoC} \max }$. 
2. Improving the $\mathrm{CoC}$ method by considering the effect of the transistor that is going to its "off" state.

3. Obtaining $L_{\mathrm{CoC} \max }$ for more complex combinations of transistors.

4. Introducing standard cell libraries based on optimum channel length.

5. Developing an analytical method to find the optimum channel length of transistors in series and parallel.

6. Incorporating $L_{\mathrm{COC} \max }$ in different logic style such Pass-Transistor Logic (PTL), Complementary Pass-Transistor logic (CPL), Dual Value Logic (DVL), DCVSL, pseudo NMOS, and dynamic logic.

7. Developing a logical effort method for sub-threshold circuits based on the channel length ${ }^{6}$.

8. Studying the effect of the channel length manipulation on the energy and power consumption in detail.

9. Developing an analytical method for finding the channel length resulting in the minimum energy.

10. Investigating the effect of channel length manipulation on noise and robustness of digital logic gates operating in the sub-threshold region.

11. Investigating the optimal layout techniques for sub-threshold circuits.

12. Combining channel length manipulation with Parallel-Transistor-Stack (PTS) [82]

13. Replacing the super-threshold-based ISCAS test benches with test benches suitable for sub-threshold operation.

14. Performing Monte Carlo simulation to explore the effect of channel length on PVT variations.

15. Exploring Layout-Dependant (LOD) proximity effect on the performance of long transistors.

Finally, it is obvious that this work can be easily extended to design various ULP analog circuits operating in the sub-threshold region.

\footnotetext{
${ }^{6}$ conventional logical effort is based on the channel width
} 


\section{List of References}

[1] J. Burr and A. Peterson, "Energy Consideration in Multiple-Module Based Multiprocessors," in IEEE Conference on Computer Design Digest for Technical Papers, pp. 593-600, 1991.

[2] J.-J. Kim and K. Roy, "Double Gate-MOSFET Sub-threshold Circuit for Ultra-LowPower Applications," IEEE Transactions on Electron Devices, vol. 51, no. 9, pp. 1468-1474, 2004.

[3] V. De and S. Borkar, "Technology and Design Challenges for Low-Power and Highperformance Microprocessors," International Symposium on Low-power Electronics and Design, pp. 163-168, 1999.

[4] J. Kwong, Y. Ramadass, N. Verma, M. Koesler, H. Moorman, and A. P. Chandrakasan, "A $65 \mathrm{~nm}$ sub-Vt microcontoller with integrated SRAM and Switched-capacitor DC-DC Converter," IEEE Intl. Solid-State Circuits Conference, pp. 318-319, 2008.

[5] H. Soeleman and K. Roy, "Digital CMOS Logic Operation in the Sub-Threshold Region," Department of Electrical and Computer Engineering, West Lafayette, IN 47907, USA, 2000.

[6] M. Muker, "Sub-Threshold CMOS Logic Design Using Parallel Transistor Stacks," in Masters' thesis, Dept. Electronics, Carleton University, 2010.

[7] M. Nabvi, "Designing Faster CMOS Sub-threshold Circuits Using Transistor Sizing and Parallel Transitor Stacks," in Masters' Thesis, Dept, Electronics, Carleton University, 2012.

[8] T.-H. Kim, J. Keane, H. Eom, and C. H. Kim, "Utilizing Reverse-Short-ChannelEffect for Optimal Subthreshold Circuit Design," IEEE Transaction on VLSI Systems, pp. 821-829, JULY 2007.

[9] S. Keller, S. Siddharth, C. Moore and A. J. Martin, "Reliable Minimum-Energy CMOS Circuit Design," in Varill 2nd European Workshop on CMOS Variability, 2011. 
[10] S. Hanson, M. Seok, D. Sylvester and D. Blaauw, "Nanometer Device Scaling in Subthreshold Logic and SRAM," IEEE Transaction on Electron Devices, vol. 55, no. 1, pp. 175-185, 2008.

[11] D. Bol, R. Ambroise, D. Flandre and J.-D. Legat, "Channel Length Upsize for Robust and Compact Sub-threshold SRAM," Microelectronics laboratory, Universite catholique de Louvain, Louvain-la-Neuve, Belgium, 2011.

[12] "An interview with Jack Kilby," Texas Instrument, available at www.ti.com/corp/dpcs/kilbyctr/interview.shtml.

[13] G. E. Moore, "Cramming More Components onto Itegrated Circuits," Electronics, vol. 38 , no. 8, pp. 114-117, 1965.

[14] R. Dennard et. al, "Design of Ion-implanted MOSFETs with Very Small Physical Dimensions," Journal of Solid State Circuits , vol. 9, no. 5, pp. 256-268, 1974.

[15] N. H. E. Weste and D. M. Harris, CMOS VLSI Design, A Circuits and Systems Perspective, Toronto: Addison-Wesley, 2011.

[16] "List of CPU power dissipation figures," available at http://en.wikipedia.org/wiki/List_of_CPU_power_dissipation_figures.

[17] Y. Pu, "On the Road towards Robust and Ultra Low Energy CMOS Digital Circuits Using Sub/Near Threshold Power Supply," PhD Thesis Dissertation, Eindhoven University of Technology, 2009.

[18] C. Piguet, "History of Low-power Electronics," in Low-Power Electronics Design, pp. CRC Press 11-15, 2005.

[19] A. P. Chandrakasan and R. W. Broederson, Low-power CMOS Desin, Wiely-IEEE Press, January 1998.

[20] R. Wieinstein, "RFID: A Technical Overview and Its Application to the Enterprise," IT Professional, vol. 7, no. 3, pp. 27-33, 2005.

[21] A. Mainwaring, J. Polastre, R. Szewczyk, D. Culler and J. Anderson, "Wireless Sensor Networks for Habitat Monitoring," ACM International Workshop on Wireless Sensor Networks and Applications (WSNA), pp. 88-97, 2002.

[22] "[Online]. Available at http://www.alertsystems.org/". 
[23] K. Chintalapudi, E. Johnson and R. Govindan, "Structural Damage Detection Using Wireless Sensor-Actuator Networks," in Proceedings of the IEEE International Symposium on Intelligent Control, 2005.

[24] L. Schwiebert, S. Gupta and J. Wienmann, "Reseach Challange in Wireless Networks of Biomedical Sensors," Mobile Computing and Networking, pp. 151-165, 2001.

[25] I. Korhonen, J. Parkka and M. Van Gils, "Health Monitoring in the Home of the Future," IEEE Engineering Mediciene and Biology, vol. 22, no. 3, pp. 66-73, 2003.

[26] R. Hahn and H. Reichel, "Batteries and Power Supplies for Wearable and Ubiquitous Computing," in Proc. 3rd Int. Symp. Wearable Computers, pp 168-169, 1999.

[27] M. Hempstead, N. Tripathi, P. Mauro, G. Wei and D. Brooks, "An Ultra-Low-Power System Architecture for Sensor Network Applications," in Proc. Intl. Symp. on Computer Architecture , pp. 208-219, 2005.

[28] H. Kulah and K. Najafi, "An Electromagnetic Micro Power Generator for LowFrequency Environment Vibrations," in Proc. of the IEEE Intl. Conf. for Micro Elctro-Mechanical Systems, pp 237-240, 2004.

[29] S. Roundy, P. Wright and J. Rabbaey, "A Study of Low Level Vibrations as a Power Source for Wireless Sensor Nodes," in Computer Communications, vol.26, no. 11, pp. 1131-1144, 2003.

[30] S. Meninger, J. Mur-Miranda, R. Amirtharajah, A. Chandrakasan and J. Lang, "Vibration-to-Electric Energy Conversion," IEEE transaction on Very Large Scale Integration (VLSI), vol. 9, no. 1, pp. 64-76, 2001.

[31] H. Bottner, J. Nurnus, A. Gavrikov, G. Kuhner, M. Jagle, C. Kunzel, D. Eberhard, G. Plescher, A. Schubert and K.-H. Schlereth, "New Thermostatic Component Using Microsystems Technologies," IEEE/ASME Journal of Microelectronics Systems, vol. 13, no. 3, pp. 414-420, 2004.

[32] "Panasonic Solar Cells Technical Handbook '98/'99," Matsushita Battery Industrial Co., Ltd., Aug. 1998. 
[33] R. M. Swanson and J. D. Meindl, "Ion-implanted compllementary MOS transistors in low-voltage circuits," IEEE Journal of Solid State Circuits (JSCC), vol. 7, no. 2, pp. 146-153, 1972.

[34] R. Swanson, "Complementary MOS Transistors in Micropower Circuits," PhD dissertation, Stanford University, 1974.

[35] B. Zhai, D. Blaauw, D. Sylvester and K. Flautner, "Theoretical and Practical Limits of Dynamic Voltage Scaling," DAC, pp. 7-11, 2004.

[36] B. H. Calhoun, A. Wang and A. Chandrakasan, "Modeling and Sizing for Minimum Energy Operation in Subthreshold Circuits," IEEE Journal of Solid State Circuits, vol. 40, no. 9, pp. 1778-1786, Sep 2005.

[37] J. T. Kao, M. Masayuki and A. P. Chandrakasn, "A 175-mV Multiply-accumulate Unit Using an Adaptive Supply Voltage and Body-Bias Architecture," IEEE Journal of Solid State Circuits, vol. 37, no. 11, pp. 1545-1554, 2002.

[38] E. A. Vittoz and J. Fellarth, "New Analog CMOS IC's Based on Weak-inversion Operation," in European Solid-State Circuits Conference, pp. 12-13, 1976.

[39] E. A. Vittoz, "Origin of Weak-inversion (or sub-threshold) Circuit Design," in Subthreshold Design for Ultra-Low-Power Systems, A. P. Chandrakasan, Springer, 2005, pp. 11-23.

[40] H. Soeleman and K. Roy, "Ultra-low Power Digital Sub-threshold Logic Circuits," in Proc IEEE/ACM Intl. Symp. Low-Power Electronics and Design, pp. 94-96, 1999.

[41] J. Wang and B. H. Calhoun, "Techniques to Extend Canary-based Standby VDD Scaling for SRAMs to $45 \mathrm{~nm}$ and beyond," IEEE Journal of Solid State Circuits, vol. 43, no. 11, pp. 2514-2523, 2008.

[42] B. H. Calhoun and A. Chandrakasan, "Ultra-Dynamic Voltage Scaling Using Subthreshold Operation and Local Voltage Dithering," IEEE Journal of Solid State Circuits, vol. 41, no. 1, pp. 238-245, 2006.

[43] B. H. Calhoun et al., "Flexible Circuits and Architecture for Ultra-Low-Power," in Proceeding IEEE, vol. 98, no. 2, pp. 267-282, 2010.

[44] B. Zhai, R. G. Dresliniski, D. Blaauw, T. Mudge and D. Sylvester, "Energy Efficient 
Near-threshold Chip Multi-processing," Proceeding IEEE/ACM Intl. Symposium Low-power Electronics and Design, pp. 32-37, 2007.

[45] H. Lee, Y. J. Park, H. S. Min, H. Shin and D. Kang, "Reduction of Reverse-ShortChannel-Effect in High-Energy Implanted Retrograde Well," Journal of the Korean Physical Society, vol. 40, no. 4, pp. 629-652, 2002.

[46] K. Roy, S. Mukhopadhhyay and H. Mahmoodi-Meimand, "Leakage Current Mechanisms and Leakage Reduction Techniques in Deep-Submicrometer CMOS Circuits," in Proceedings of The IEEE, 2003.

[47] B. C. Paul, A. Raychowdhury and K. Roy, "Device Optimization for Digital Subthreshold Logic," IEEE Transactions on Electron Devices, vol. 52, no. 2, pp. 237$247,2005$.

[48] S. Luan, H. Liu, R. Jia and J. Wang, "Two-dimensional Sub-threshold Current Model for Dual-material Gate SOI nMOSFETs with Single Halo," Front. Electr. Electron. Eng. China, pp. 98-103, 2009.

[49] A. Massimo, "Understanding DC Behavior of Subthreshold CMOS Logic Through Closed-Form Analysis," IEEE Tranasactions on Circuits and Systems, pp. 15971607, July 2010.

[50] D. M. Harris, B. Keller, J. Karl and S. Keller, "A Transregional Model for NearThreshold Circuits with Application to Minimum-energy Operation," in 22nd International Conference on Microelectronics (ICM 2010), , 2010.

[51] J. Meindel and J. A. Davis, "The Fundemental Limit on Binary Switching Energy for Terascale Integration (TSI)," IEEE Journal of Soild State Circuits, vol. 35, no. 10, pp. 1515-1516, 2000.

[52] M.Muker and M. Shams, "Designing Digital Sub-threshold CMOS Circuits Using Paralle Transistor Stacks," Electronics Letters, vol. 47, no. No. 6, 2011.

[53] M.Nabavi and M. Shams, "A Gate Sizing and Transistor Fingering Strategy for Subthreshold CMOS Circuits," IEICE Journal of Electronics Express (ELEX), accepted 2012.

[54] J.Zhou, S. Jayapal, J. Stuyt, J. Huisken and H. de Groot, "The Impact of Inverse- 
Narrow-Width-Eeffect on Sub-threshold Device Sizing," IEEE DAC, pp. 267-272, 2011.

[55] H.K.O.Berge and S. Aunet, "Benefits of Decomposing Wide CMOS Transistors into Minimum-size Gates," NORCHIP, pp. 1-4, 2009.

[56] J. Keane, H. Eom, T. H. Kim, S. Sapatnekar and C. Kim, "Sub-threshold Logical Effort: A Systematic Framework for Optimal Sub-threshold Device Sizing," in $D A C^{\prime} 06$, San Fransisco, California, USA, 2006.

[57] C. Giacomotto and V. G. Oklobdžija, "Logic Style Comparison for Ultra Low Power Applications," Advanced Computer Systems Engineering Laboratory, Department of Electrical and Computer Engineering, University of California, Davis, CA 95616.

[58] H. Soeleman, K. Roy and B. C. Paul, "Robust Sub-threshold Logic for Ultra-LowPower Operation," IEEE Transactions on Very Large Scale Integration, vol. 9, no. 1, pp. 90-99, 2001.

[59] A. Pajkanovic, T. Kazmierski and B. Dokic, "Adiabatic Digital Circuits Based on Sub-threshold Operation of Pass-transistor and Slowly Ramping Signals," in Proceedings of Small Systems Simulation Symposium, Niš, Serbia, 2012.

[60] J. Chen, L. T. Clark and Y. Cao, "Robust Design of High Fan-In/Out Sub-threshold Circuits," in International Conference on Computer Design (ICCD'05), 2005.

[61] M. R. Bagheri, "Ultra Low Power Sub-threshold Bridge Style Adder in Nanometer Technologies," Canadian Journal on Electrical and Electronics Engineering, vol. 2, no. 7, pp. 294-299, 2011.

[62] A. Tajalli, E. Brauer, Y. Leblebici and E. Vittoz, "Sub-threshold Source-Coupled Logic Circuits for Ultra-Low-Power Applications," IEEE Transactions of Solid-state Circuits, vol. 43, no. 7, pp. 1699-1710, 2009.

[63] J. Nyathi and B. Bero, "Logic Circuits Operating in Sub-threshold Voltages," in ISLPED'06, Tegemsee, Germany, 2006.

[64] N. Verma, J. Kwong and A. P. Chandrakasan, "Nanometer MOSFET Variation in Minimum-Energy Sub-threshold Circuits," IEEE Transactions on Electron Devices, vol. 55, no. 1, pp. 163-174, 2008. 
[65] A. Wang and A. P. Cahndrakasan, "Optimal Supply and Threshold Scaling for Subthreshold CMOS Circuits," Proceedings of the IEEE Computer Society Annual Symposium on VLSI (ISVLSI'02), 2002.

[66] D. Bol, "Robust and Energy-Efficient Ultra-Low-Voltage Circuit Design under Timing Constraints in 65/45 nm CMOS," Journal of Low Power Electronics and Applications, pp. 1-19, 2011.

[67] D. Bol, D. Kamel, D. Flandre and J.-D. Legat, "Nanometer MOSFET Effects on the Minimum-Energy Point of 45nm Sub-threshold Logic," in ISLPED'09, San Francisco, California, USA, 2009.

[68] D. Bol, R. Ambroise, D. Flandre and J.-D. Legat, "Impact of Technology Scaling on Digital Sub-threshold Circuits," IEEE Computer Society Anmual Symposium on VLSI, pp. 179-184, 2008.

[69] B. Calhoun and A. P. Cahndarkasan, "Characterizing and Modeling Minimum Energy Operation for Sub-threshold Circuits," in ISLPED'04, Newport Beach, California, USA, 2004.

[70] J. Chen, L. T. Clark and T. H. Chen, "An Ultra-Low-Power Memory with a Subthreshold Power Supply Voltage," IEEE Journal of Solid State Circuits, vol. 41, no. 10, pp. 2344-2353, 2006.

[71] B. H. Calhoun and A. P. Chandrakasan, "A $256 \mathrm{~kb}$ Sub-threshold SRAM in $65 \mathrm{~nm}$ CMOS," in ISSCC'06, pp. 2592-2601, San Francisco, California, USA, 2006.

[72] G. Wang and et al., "A $0.127 \mathrm{um}^{\wedge} 2$ high performance $65 \mathrm{~nm}$ SOI based embedded DRAM for on-processor applications," in Proc. Intl. Electron Device Meeting, 2006.

[73] J. Zhou, S. Jayapal, B. Busze, L. Huang and J. Stuyt, "A $40 \mathrm{~nm}$ Inverse-NarrowWidth-Effect-Aware Sub-Threshold Standard Cell Library," in DAC'11, June 5-10, 2011, San Diego, California, USA.

[74] P. Xiaonan Zhang, "Designing Low-power Standard Cell Library With Improved Drive Granularity," Qualcomm availabale at "www.design-reuse.com".

[75] W. Shockley, "A unipolar field effect transistor," in Proc. IRE, 1952.

[76] T. Sakurai and A. R. Newton, "Alpha-Power-Law MOSFET Model and Its 
Application to CMOS Inverter and other Fomulas," IEEE Journal of Solid State Circuits, vol. 25, pp. 584-594, Apr 1990.

[77] B. Razavi, Design of Analog CMOS Integrated Circuits, Boston: Mc Graw Hill, 2001.

[78] A. B. Bhattacharyya, Compact MOSFET Models for VLSI Design, Asia: John Wiley \& Sons, 2009.

[79] D. W.Greve, Field Effect Devices and Aplications, London: Printice-Hall, 1998.

[80] Y.Tisividis, Operation and Modeling of the MOS Transistors, Boston: McGraw-Hill, 1999.

[81] J. M. Rabaey, A. Chandrakasan and B. Nikolic, Digital Integrated Circuits, A Design Perspective, London: Prentice Hall, 2003.

[82] A. Bellaouar and M. I. Elmasry, Low-Power Digital VLSI Design, Massachusetts: Kluwer Academic Publishers, 1995. 